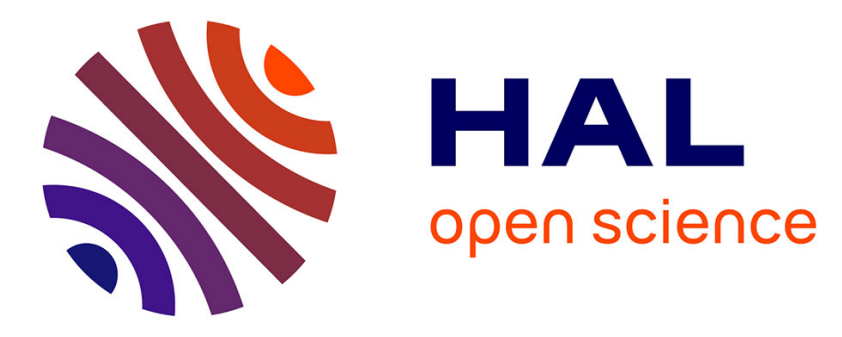

\title{
Mathematical analysis of guided water waves
}

Anne-Sophie Bonnet-Ben Dhia, Patrick Joly

\section{To cite this version:}

Anne-Sophie Bonnet-Ben Dhia, Patrick Joly. Mathematical analysis of guided water waves. [Research

Report] RR-1629, INRIA. 1992. inria-00074932

\section{HAL Id: inria-00074932 \\ https://hal.inria.fr/inria-00074932}

Submitted on 24 May 2006

HAL is a multi-disciplinary open access archive for the deposit and dissemination of scientific research documents, whether they are published or not. The documents may come from teaching and research institutions in France or abroad, or from public or private research centers.
L'archive ouverte pluridisciplinaire HAL, est destinée au dépôt et à la diffusion de documents scientifiques de niveau recherche, publiés ou non, émanant des établissements d'enseignement et de recherche français ou étrangers, des laboratoires publics ou privés. 


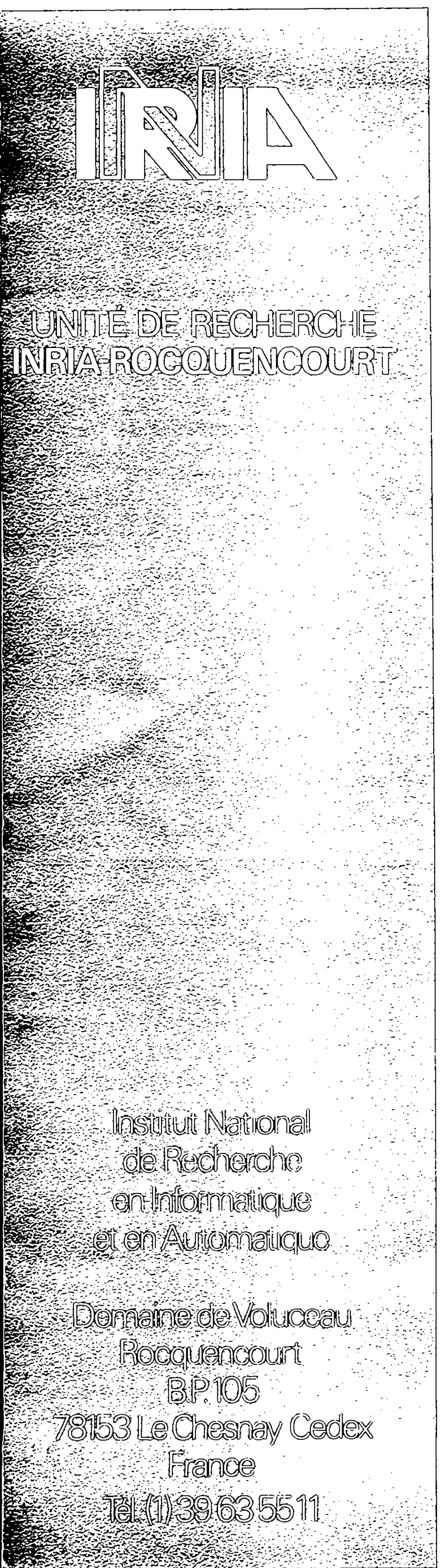

Rapports de Recherche

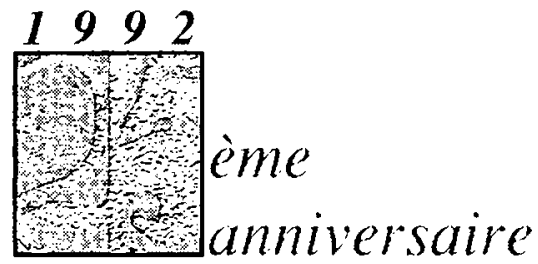

$\mathrm{N}^{\circ} 1629$

\title{
Programme 6
}

Calcul Scientifique, Modélisation et

Logiciel numérique par Ordinateur

\section{MATHEMATICAL ANALYSIS OF GUIDED WATER WAVES}

\author{
Anne-Sophie BONNET-BEN DHIA \\ Patrick JOLY
}

Février 1992 


\section{Contents}

1 Position of the problem 2

2 Presentation of the content of the paper 5

2.1 The state of the art .................. 5

2.2 The main results .................... 6

3 Mathematical formulations of the problem $\quad 10$

3.1 The eigenvalue problem . . . . . . . . . . . . . . . . 10

3.2 An equivalent formulation in a bounded domain . . . . . . . . . 11

4 The basic spectral theory of the operator $A(\omega) \quad 17$

4.1 Selfadjointness of $A(\omega)$ and lower bound for the spectrum . . . . . . . . . . 17

4.2 The essential spectrum . . . . . . . . . . . . . . . . . . . 21

4.3 On the existence of eigenvalues in the essential spectrum ........ 23

4.4 The Min - Max principle . . . . . . . . . . . . . 26

5 Mathematical study of the dispersion curves 28

5.1 Preliminary results . . . . . . . . . . . . . . . . . . 28

5.1.1 General properties of the functions $S_{m}(\omega) \ldots \ldots 28$

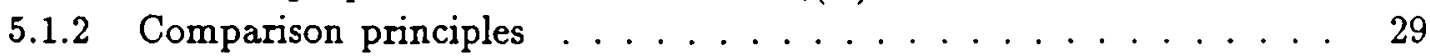

5.2 Results for abitrary shapes . . . . . . . . . . . . . . . 33

5.2 .1 Existence results ..................... 33

5.2.2 Properties of the fundamental mode . . . . . . . . . . . . . 38

5.3 The case of the cliff . . . . . . . . . . . . . . . . . . 39

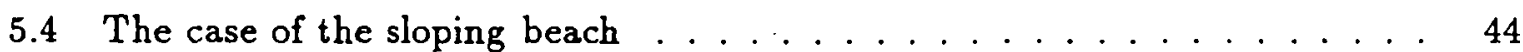

5.5 The case of the tangential beach . . . . . . . . . . . . . 52

6 The numerical approach $\quad \mathbf{5 7}$

6.1 The numerical method . . . . . . . . . . . . . . . . 57

6.1 .1 The discretization . . . . . . . . . . . . . . 57

6.1.2 A non linear eigenvalue problem ............ 58

6.1.3 The algorithm .................. 59

6.2 Numerical results . . . . . . . . . . . . . . . . . 59

6.2 .1 The case of a cliff ................ 60

6.2.2 The case of a sloping beach . . . . . . . . . . . . . . 61

$\begin{array}{ll}\text { Annex } & 64\end{array}$

$\begin{array}{ll}\text { References } & 67\end{array}$ 


\section{Position of the problem}

The mathematical model we shall consider in this paper can be described with the help of the equations governing the small displacements associated to the irrotational flow of a perfect incompressible fluid. These equations are derived by the linearization of the classical fluid dynamics equations ([26], [33]). It is in particular valid for the description of the water waves (or gravity waves) in finite depth oceans. For the particular purpose we follow here, we shall suppose that the fluid occupies a cylindrical domain of the form $\Omega \times \mathbf{R}$ where $\Omega$ denotes an unbounded open set of $\mathbf{R}^{2}$ defined by :

$$
\Omega=\left\{(x, y) \in \mathbf{R}^{2} /-h(x)<y<0, x>0\right\}
$$

where $h(x)$ is a positive function. We shall not make any regularity assumption about $h(x)$ and only assume that $\Omega$ is a connected open set. Nevertheless, we shall assume that $h(x)$ has the following properties:

(i) $h(x)$ is measurable and bounded

(ii) $h(x)=h_{\infty}$ for $x>x_{0} \quad\left(x_{0}>0, h_{\infty}>0\right)$

and shall add in the sequel some particular hypotheses on the behaviour of $h(x)$ in some neighbourhood of the origin, depending of the nature of the result we wish to point out.

We shall denote by $(x, y, z)$ the generic point of the domain. Our assumptions permit us to describe the case of an infinitely long coast which is invariant under any translation in the horizontal $z$ direction. The direction $y<0$ corresponds to the depth. The fact that $h(x)$ is bounded corresponds to the fact that we consider finite depth oceans, as illustrated in the figure below.

We shall decompose the boundary of $\Omega$ as $\partial \Omega=\Gamma_{F} \cup \Gamma_{B}$ where :

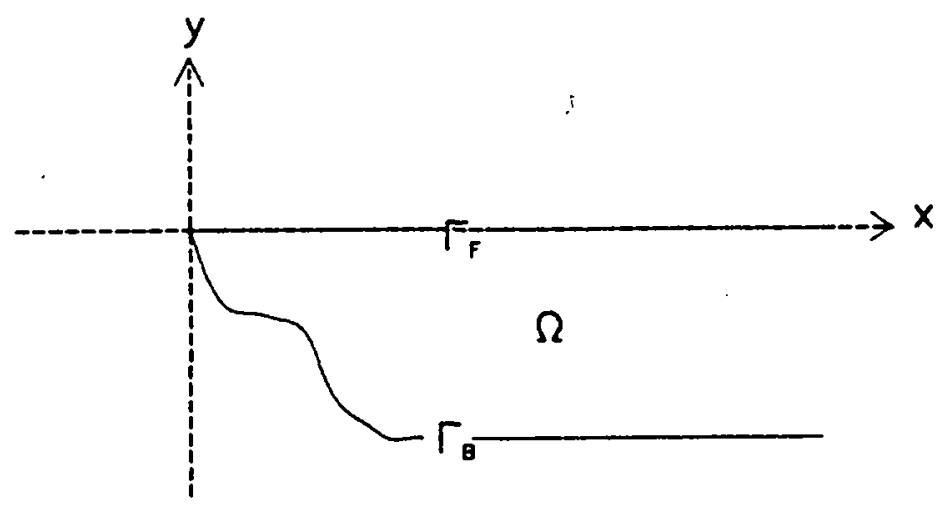

Figure 1: The cross section

$\Gamma_{F}=\{(x, 0), x>0\}$ is the mean free surface

$\Gamma_{B}=\partial \Omega-\Gamma_{F}$ is the bottom of the ocean 
The equations of the model are

$$
\begin{gathered}
\frac{\partial^{2} \phi}{\partial x^{2}}+\frac{\partial^{2} \phi}{\partial y^{2}}+\frac{\partial^{2} \phi}{\partial z^{2}}=0 \text { in } \Omega \times \mathbf{R} \\
\frac{\partial^{2} \phi}{\partial t^{2}}+g \frac{\partial \phi}{\partial y}=0 \quad \text { on } \Gamma_{F} \times \mathbf{R} \\
\frac{\partial \phi}{\partial n}=0 \quad \text { on } \Gamma_{B} \times \mathbb{R}
\end{gathered}
$$

Here, $\phi$ denotes the velocity potential in the fluid, which means that the velocity $v(x, z, t)$ of a particle located at point $(x, y, z)$ at time $t$ is given by :

$$
v(x, y, z, t)=\nabla \phi(x, y, z, t)
$$

This expresses that we consider an irrotational flow. Equation (1.2) expresses the incompressibility of the fluid ( $\operatorname{div} v=0$ ) while the boundary equation (1.3) is the free surface condition. This equation is responsible for wave propagation. The Neumann boundary condition (1.4) expresses that the bottom of the ocean does not move. The gravity acceleration, which is denoted by $\mathrm{g}$, will be assumed to be equal to 1 , which is valid up to an appropriate scaling in space variables. In the present work we are interested in guided waves or trapped modes, i.e. particular solutions in the form :

$$
\phi(x, y, z, t)=\Re e\left\{\varphi(x, y) e^{i(\omega t-\beta z)}\right\}
$$

where $(\omega, \beta)$ are real numbers and $\varphi(x, y)$ is a real valued function for which we require the "localization property" :

$$
\int_{\Omega}\left(|\varphi(x, y)|^{2}+|\nabla \varphi(x, y)|^{2}\right) d x d y<+\infty
$$

Such a solution corresponds to an harmonic wave propagating in the z-direction without any attenuation or distorsion. $\omega$ is the pulsation, $\beta$ the wave number. The wave propagates with the phase velocity $\omega / \beta$. The condition (1.7) expresses the fact that the transverse energy of the wave is finite and in fact confined, as we shall see later, in some neighbourhood of the coast.

Let us point out that such solutions can exist if and only if $\omega$ and $\beta$ are linked by a certain relation which is the "dispersion relation" of the mode. The main objective of this paper is to deal with the problem of the existence and the properties of these modes, with a particular attention devoted to the low frequency (small values of $\beta$ ) and high frequency (great values of $\beta$ ) analyses. One of the interests of our results is to point out a large variety of qualitative results depending essentially on the geometry of the coast. From this point of view, the water waves model appears as richer than other classical model of wave propagation previously studied in the mathematical literature. 
The outline of our paper is as follows : in section 2.1 we give a brief survey of results previously obtained on the subject. Most of our results will appear as various extensions and generalizations of these results. They will be presented in section 2.2 . In section 3 . we give the theoretical framework of our study and a mathematical formulation of the problem. Some preliminary results are established in section 4 . The main theorems of this article are stated and proved in section 5. Finally, in section 6 ., we describe a numerical method for the computation of these guided waves and present various numerical results illustrating the theory. 


\section{Presentation of the content of the paper}

\subsection{The state of the art}

The question we intend to address in the paper has already been extensively studied and discussed in the mathematical literature. Stokes [27], in 1846, was the first to show the existence of the phenomenon of trapped modes by exhibiting a particular analytical solution in the case of the plane sloping beach (see figure 2) It is only in 1952 that Ursell [30]

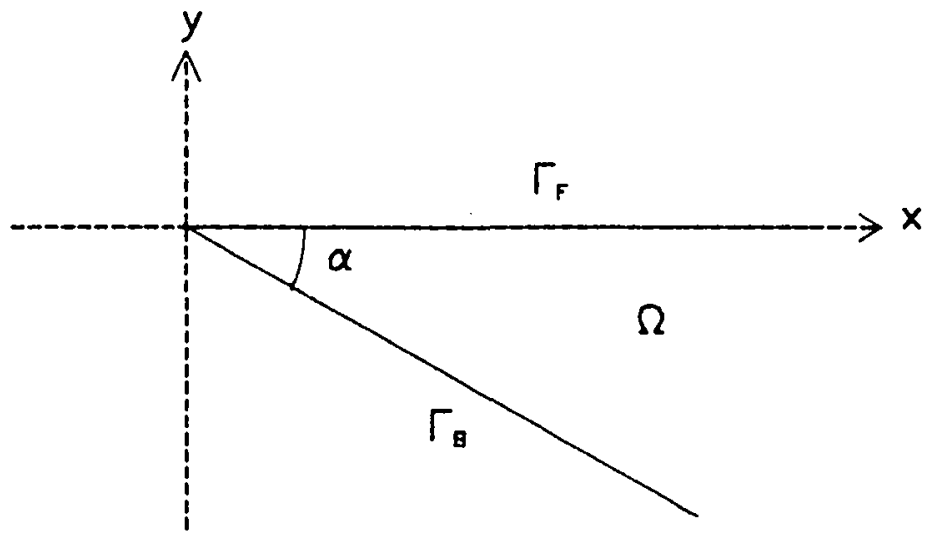

Figure 2: The plane sloping beach

showed that, at least for sufficiently small values of the angle $\alpha$, the Stokes' solution was the first of a finite number $M(\alpha)$ of such trapped modes. This number $M(\alpha)$ is the largest integer $n$ for which $(2 n-1) \alpha \leq \frac{\pi}{2}$ ( let us note that Ursell did not prove that he got all the trapped modes associated with the plane sloping beach model). Ursell also pointed out [29] (see also [20]) that the guided wave phenomenon also occured in the case of an infinitely long submerged cylinder in an infinite half space. Nevertheless these situations were slightly different than ours since we consider only oceans of finite depth.

In fact it seems that this is Jones [14] who, at the period of the initial developments of modern linear functional analysis, especially spectral theory, obtained the first mathematical results concerning the specific problem we treat here. Dealing with the study of the spectral analysis for the Laplace operator in unbounded domains with various boundary conditions, he stated in particular the "comparison theorems" (see section 5.1.2) which lead to essential results in the theory of guided gravity waves as the finiteness of modes for a given value of $\beta$. The first general existence result was obtained by Garipov in 1965 (see [16] ). Within the framework of the modern spectral theory (that we shall adopt in this paper) he showed that, at least when $\beta$ is large enough, there exists a guided wave under the only assumption that the function $h(x)$ presents a strict local minimum (see theorem 5.1 of section 5 of the present paper). This mode, known as the fundamental mode, was studied in more details by Grimshaw in $1974[10]$, with a particular attention to low frequencies. Thanks to comparison theorems of a different kind from Jones'ones, 
he obtained upper and lower bounds for the dispersion relation of this mode and found a necessary condition for this wave to exist for any value of $\beta$. He also obtained, by the way of integral equations, the dispersion relation of guided waves for the case of a shelf.

This specific case was treated in greater depth by Evans and Mc Iver ([8]), using a slightly different approach. They proved that the fundamental mode was the only one for small and large value of $\beta$. Moreover they obtained, in relation with the initial work of Jones, sharp estimates for the number of guided waves for a given value of $\beta$, that they completed with the help of numerical results. Recently,in 1987 ( $[31]$ ), Ursell revisited the works of Jones and Garipov with the help of simpler arguments. He uses in particular the so-called Kelvin's theorem which is nothing but a specific version of the general Max - Min principle [23] we shall use extensively in this article.

\subsection{The main results}

In order to give a clear overview of the content of our paper we have chosen to present in this subsection a panorama of the main results we have obtained. Nevertheless, we shall not give the precise assumptions and statements of the theorems. These will be given, together with the proofs, in section 5 .

Our results can be separated into two parts as follows:

- the general results, which are independent on the particular shape of the coast i.e. the behaviour of $h(x)$ near $x=0$.

- the specific results wich are directly related to the geometry of the coast.

To describe these results we shall need the following notation:

$$
\left\{\begin{array}{l}
h^{+}=\text {ess } \sup _{x \in \mathbf{R}^{+}} h(x) \\
h^{-}=\text {ess } \inf _{x \in \mathbf{R}^{+}} h(x) \\
h_{\infty}=h(x) \text { for } x>x_{0}
\end{array}\right.
$$

Moreover we shall denote by $\beta=\beta_{\infty}(\omega)$ the unique solution of the transcendental equation

$$
\beta \tanh \beta h_{\infty}=\omega^{2}, \beta>0,
$$

and what we shall call a guided wave in this section will be a solution of the form (1.6), satisfying the condition (1.7) and the additional inequality :

$$
\beta>\beta_{\infty}(\omega) .
$$

This condition is sometimes considered as automatically satisfied in the literature. In particular all the results we spoke about in section 2.1 suppose implicitly this condition. We shall come back very briefly in section 4.3 on the question of the existence of possible guided modes for which (2.3) does not hold.

(i) The general results 
1. For large $\beta$, there exists at least one guided wave, the fundamental mode, as soon as $h^{-}<h_{\infty}$.

2. For any value of $\beta$, the number of guided waves is finite.

3. For small $\beta$, there exists at most one guided wave.

4. The fundamental mode exists for any $\beta$ if and only if one has :

$$
\int_{0}^{+\infty}\left(h(x)-h_{\infty}\right) d x<+\infty
$$

5. The function $\beta \rightarrow \omega(\beta)$ describing the dispersion relation of a guided wave is increasing.

6. If $h^{+}=h_{\infty}$ no guided waves exist.

The existence result (1) is the one of Garipov [16] and is also given by Ursell in [31]. Result (2) initially due to Jones [14], is also stated in [31] .Our result (3) seems to be new. The condition (2.4) of result (4) was found by Grimshaw [10] as a necessary condition for the existence of a trapped mode at low frequencies. Result (5) is a simple monotonicity results which expresses that the group velocity $\frac{d \omega}{d \beta}$ of a guided wave is positive. (6) shows that the condition $h^{-}<h_{\infty}$ is a necessary and sufficient condition for the existence of guided waves

(ii) The specific results

These results concern essentially the high frequency analysis and depend on the behaviour of $h(x)$ at $x=0$. We shall consider three types of geometries :

1 - the cliff $\Longleftrightarrow h(0)>0$ or $\left\{h(0)=0, h^{\prime}(0)=+\infty\right\}$ (cf figure 3 )
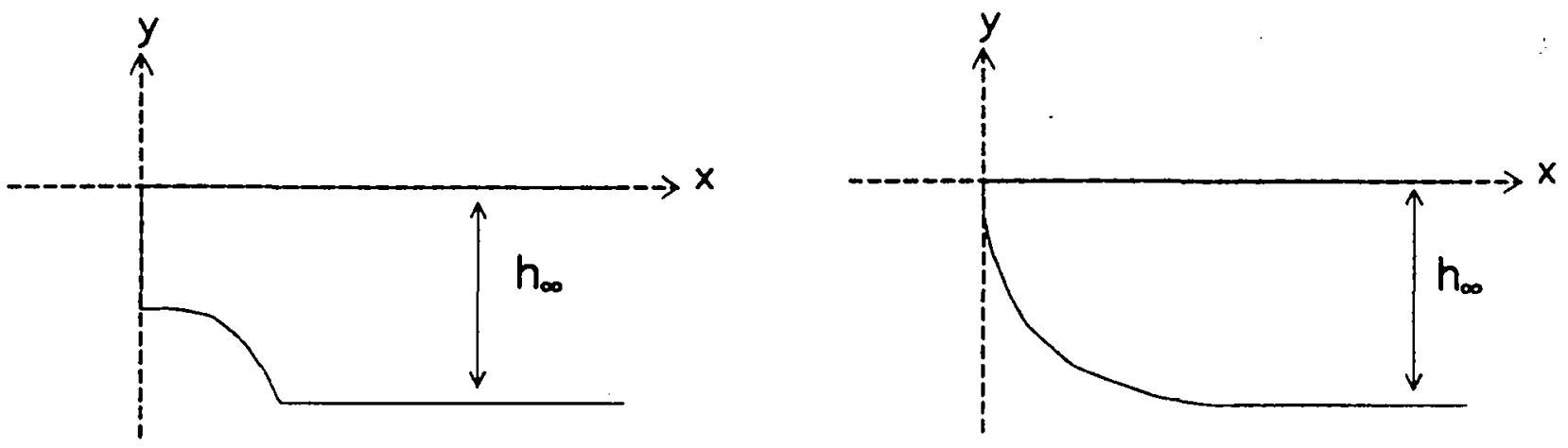

Figure 3: Two examples of a cliff

2 - the sloping beach $\Longleftrightarrow h(x) \sim x \tan \alpha \quad(x \rightarrow 0)$ (cf figure 4) 


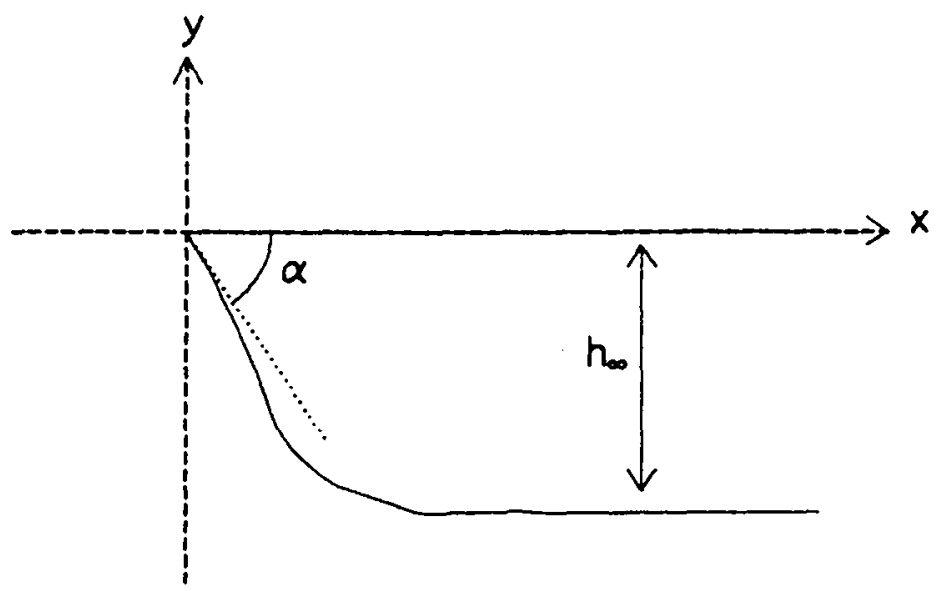

Figure 4: The case of a sloping beach

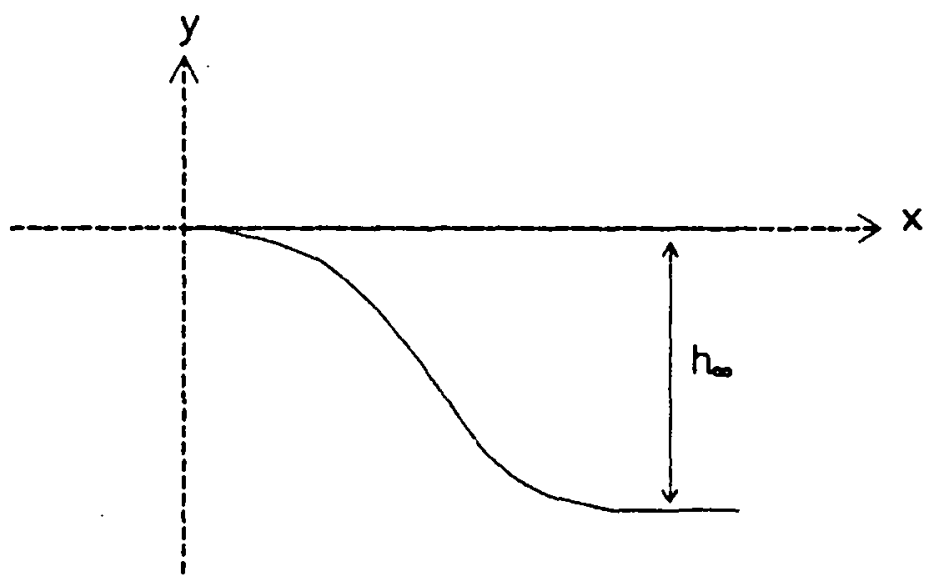

Figure 5: The tangential beach 
3 - the tangential beach $\Longleftrightarrow h(0)=0, h^{\prime}(0)=0$ (cf figure 5)

The corresponding results are the following :

1. In the case of the cliff, there exists at most one guided wave at large frequencies .

2. In the case of the sloping beach, when $\beta$ is large enough, there exist at least $M(\alpha)$ guided waves at large frequencies, where $N(\alpha)$ is the greatest integer $n$ for which $(2 n-1) \alpha \leq \frac{\pi}{2}$

3. In the case of the tangential beach, the number of guided waves is unbounded when $\beta$ goes to infinity. More precisely there exists an increasing sequence of real numbers $\beta_{m}$, the thresholds, such that there exist at least $m$ guided waves as soon as $\beta$ is greater than $\beta_{m}$

Result (1) appears as a generalization of the result obtained by Evans and Mc Iver in the case of the shelf. Results (2) and (3) appear as completely new. (2) refers explicity to the work of Ursell [30] while (3) was previously conjectured by the same author ([29],[31]). The situation described by this result, although it can seem complicated in the present context, is in fact similar to the one encountered in more classical problems of open waveguides ([1], $[2],[3],[4],[11],[19],[34])$. Of course, a lot of questions remain open. We shall mention some of them in the conclusion. 


\section{Mathematical formulations of the problem}

\subsection{The eigenvalue problem}

Let $\omega$ be a positive real number, we set :

$$
D(A(\omega))=\left\{\varphi \in H^{1}(\Omega) / \Delta \varphi \in L^{2}(\Omega) ;\left(\frac{\partial \varphi}{\partial y}-\omega^{2} \varphi\right)\left|\Gamma_{F}=0, \frac{\partial \varphi}{\partial n}\right|_{\Gamma_{B}}=0\right\}
$$

and introduce the unbounded operator in $L^{2}(\Omega)$, with domain $D(A(\omega))$ :

$$
\mid \begin{aligned}
& A(\omega): D(A(\omega)) \subset L^{2}(\Omega) \rightarrow L^{2}(\Omega) \\
& \varphi \rightarrow A(\omega) \varphi=-\triangle \varphi
\end{aligned}
$$

We can now make the link between the operator $A(\omega)$ and our problem. Plugging expression (1.6) into equations $(1.2),(1.3),(1.4)$, we observe that $\phi$ given by (1.6) is a guided mode if and only if the function $\varphi(x, y)$ satisfies the following set of equations :

$$
\left\{\begin{array}{l}
-\triangle \varphi=-\beta^{2} \varphi \text { in } \Omega \\
\frac{\partial \varphi}{\partial y}-\omega^{2} \varphi=0 \text { on } \Gamma_{F} \\
\frac{\partial \varphi}{\partial n}=0 \text { on } \Gamma_{B} \\
\varphi \in H^{1}(\Omega), \varphi \neq 0
\end{array}\right.
$$

This can be rewritten as :

$$
\begin{aligned}
& \varphi \in D(A(\omega)), \varphi \neq 0 \\
& A(\omega) \varphi=-\beta^{2} \varphi
\end{aligned}
$$

In other words, $\phi$, given by (1.6), is a guided wave if and only if $\varphi$ is an eigenfunction of the operator $A(\omega)$ associated to the eigenvalue $-\beta^{2}$. Therefore the problem of the existence of guided waves is equivalent to the problem of the existence of a non empty point spectrum of the operator $A(\omega)$ and we thus have to make the spectral analysis of a family of selfadjoint operators parametrized by $\omega>0$. Let us point out immediately that, as $A(\omega)$ is unbounded, this problem is not obvious. For instance in the case of the constant depth, i.e. when $h(x)=h_{\infty}$ for any $x>0$, we shall see that the spectrum of $A(\omega)$ is purely continuous and that there is no guided wave in this particular configuration. On the other hand the fact that the "transverse" geometries we consider are, because of (1.1), local perturbations of the constant depth profile will permit us to use compact perturbations techniques to study the point spectrum of $A(\omega)$. As we shall see a waveguide can be created only by a variation of the depth as a function of the distance to the coast. Our objective is to characterize if possible what kind of perturbation is responsible for the existence of guided waves. Such an analysis will require some a priori knowledge of the spectral properties of $A(\omega)$, which is the purpose of section 4 . But, first, let us present an alternative formulation of the problem. 


\subsection{An equivalent formulation in a bounded domain}

Let us decompose $\Omega$ as (see figure 6):

$$
\mid \begin{aligned}
& \bar{\Omega}=\bar{\Omega}_{e} \cup \bar{\Omega}_{i} \\
& \left.\Omega_{e}=\{(x, y) \in \Omega / x>d\}=\right] d,+\infty[\times]-h_{\infty}, 0[ \\
& \Omega_{i}=\{(x, y) \in \Omega / x<d\} \\
& \Sigma=\bar{\Omega}_{e} \cap \bar{\Omega}_{i}
\end{aligned}
$$

where $d>x_{0}$, so that, by (1.1), the depth is constant in $\Omega_{e}$. We are going to give an equivalent formulation of our problem which will be set only in the bounded "interior" domain $\Omega_{i}$. This formulation will be useful for both theoretical (see section 4 ) and numerical (see section 6) reasons.

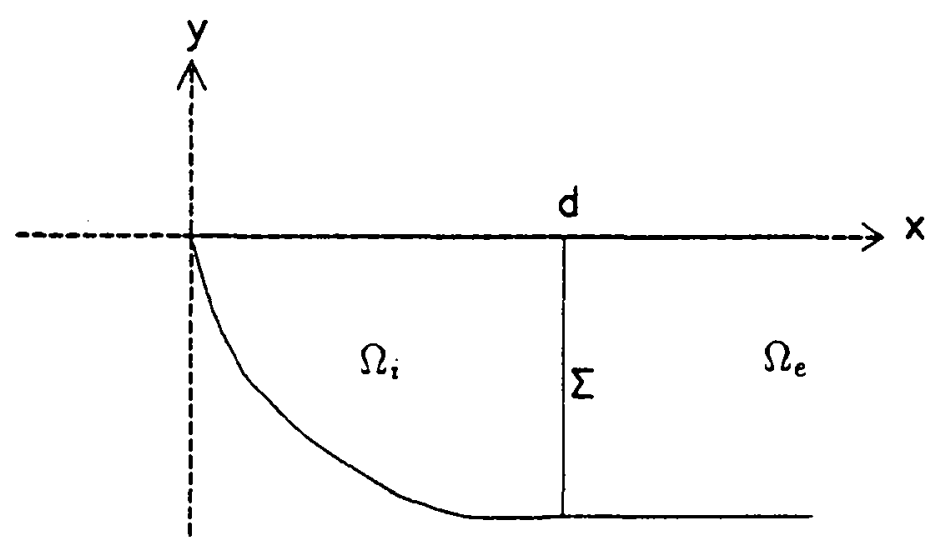

Figure 6: Decomposition of the domain $\Omega$

To establish this formulation, we will derive, for the solutions $\varphi$ of (3.3), an explicit expression of $\varphi \mid \Omega_{e}$ as an expansion over a family of functions with separated variables.

To do that, we have first to study the spectral theory of the operator $\hat{A}\left(\omega, h_{\infty}\right)$ defined as follows :

$$
\left\{\begin{array}{l}
D\left(\hat{A}\left(\omega, h_{\infty}\right)\right)=\left\{u \in H^{2}\left(-h_{\infty}, 0\right) ; \frac{d u}{d y}(0)=\omega^{2} u(0) \text { and } \frac{d u}{d y}\left(-h_{\infty}\right)=0\right\} \\
\hat{A}\left(\omega, h_{\infty}\right) u=-\frac{d^{2} u}{d y^{2}} \text { for } u \in D\left(\hat{A}\left(\omega, h_{\infty}\right)\right) .
\end{array}\right.
$$

In particular, $\hat{A}\left(\omega, h_{\infty}\right)$ can be seen as an operator acting on $\Sigma$. We have the

Lemma 3.1 The operator $\hat{A}\left(\omega, h_{\infty}\right)$ is selfadjoint, its spectrum consists in a sequence of eigenvalues $\left(\xi_{n}(\omega)\right)_{n \geq 0}$, and there exists a family of associated eigenfunctions $\left(u_{n}(\omega)\right)_{n \geq 0}$ which form an orthonormal basis of $L^{2}\left(-h_{\infty}, 0\right)$ and a total set in $H^{1}\left(-h_{\infty}, 0\right)$. 
Proof : The operator $\hat{A}\left(\omega, h_{\infty}\right)$ is associated with the following bilinear form :

$$
\hat{a}(\omega ; u, v)=\int_{-h_{\infty}}^{0} \frac{d u}{d y} \frac{d v}{d y} d y-\omega^{2} u(0) v(0)
$$

which is clearly symmetric and continuous on $H^{1}\left(-h_{\infty}, 0\right)$. Moreover there exists a constant $\mu>0$ such that the form $(u, v) \rightarrow \hat{a}(\omega ; u, v)+\mu \int_{-h_{\infty}}^{0} u v d y$ is coercive on $H^{1}\left(-h_{\infty}, 0\right)$ (this can be established easily by contradiction ). The lemma then results from the classical spectral theorem for selfadjoint operators with compact resolvant. It is a simple exercise on ordinary differential equations to determine the eigenelements of $\hat{A}\left(\omega, h_{\infty}\right)$. The result is well known (see for example Lenoir [17]):

- $\xi_{0}(\omega)=-\nu_{0}(\omega)^{2}$

where $\nu_{0}$ is equal to $\beta_{\infty}(\omega)$ which is the solution of $(2.2)$ (we just use the notation $\nu_{0}$ to simplify the expression of the series below). The associated eigenfunction is :

$$
u_{0}(\omega, y)=a_{0} \cosh \left(\nu_{0}\left(y+h_{\infty}\right)\right)
$$

with

$$
a_{0}=\left(\frac{1}{4 \nu_{0}} \sinh \left(2 \nu_{0} h_{\infty}\right)+\frac{h_{\infty}}{2}\right)^{1 / 2}
$$

- $\xi_{n}(\omega)=v_{n}(\omega)^{2}, n \geq 1$

where $\nu_{n}$ is the unique solution $\nu$ of the equation :

$$
\left\{\begin{array}{l}
\nu \tan \left(\nu h_{\infty}\right)=-\omega^{2} \\
\nu \in] n \pi-\frac{\pi}{2}, n \pi+\frac{\pi}{2}[
\end{array}\right.
$$

The associated eigenfunction is :

$$
u_{n}(\omega, y)=a_{n} \cos \left(\nu_{n}\left(y+h_{\infty}\right)\right)
$$

with

$$
a_{n}=\left(\frac{1}{4 \nu_{n}} \sin \left(2 \nu_{n} h_{\infty}\right)+\frac{h_{\infty}}{2}\right)^{1 / 2}
$$


For every $u \in L^{2}\left(-h_{\infty}, 0\right)$, we have :

$$
\|u\|_{L^{2}\left(-h_{\infty}, 0\right)}^{2}=\int_{-h_{\infty}}^{0} u^{2} d y=\sum_{n=0}^{+\infty}\left(u, u_{n}\right)^{2}
$$

where $(\cdot, \cdot)$ denotes the usual scalar product on $L^{2}\left(-h_{\infty}, 0\right)$. Likewise, we have :

$$
H^{1}\left(-h_{\infty}, 0\right)=\left\{u \in L^{2}\left(-h_{\infty}, 0\right) ; \sum_{n \geq 0} \nu_{n}^{2}(\omega)\left(u, u_{n}(\omega)\right)^{2}<+\infty\right\}
$$

and

$$
\|u\|_{1}=\left(\sum_{n=0}^{+\infty} \nu_{n}^{2}(\omega)\left(u, u_{n}(\omega)\right)^{2}\right)^{1 / 2}
$$

is a norm on $H^{1}\left(-h_{\infty}, 0\right)$, equivalent to the usual one. Moreover, by interpolation and duality, we have :

$$
H^{s}\left(-h_{\infty}, 0\right)=\left\{u=\sum_{n=0}^{+\infty} \gamma_{n} u_{n}(\omega) ; \sum_{n \geq 0} \nu_{n}(\omega)^{2 s} \gamma_{n}^{2}<+\infty\right\}
$$

for every real $s \in[-1,+1]$, and equipped with the norm

$$
\|u\|_{s}=\left(\sum_{n=0}^{+\infty} \nu_{n}(\dot{\omega})^{2 s} \gamma_{n}^{2}\right)^{1 / 2}
$$

$H^{s}\left(-h_{\infty}, 0\right)$ is the usual Sobolev space.

Remark 3.1 Since $-\beta_{\infty}^{2}(\omega)$ is the smallest eigenvalue of $\hat{A}\left(\omega, h_{\infty}\right)$, we have :

$$
\int_{-h_{\infty}}^{0}\left|\frac{d u}{d y}\right|^{2} d y-\omega^{2} u^{2}(0) \geq-\beta_{\infty}^{2}(\omega) \int_{-h_{\infty}}^{0} u^{2} d y
$$

for every $u \in H^{1}\left(-h_{\infty}, 0\right)$. This estimate will be used often in the sequel.

We are now able to establish the

Lemma 3.2 Let $\lambda \in \mathbf{R}$ and $\varphi \in H^{1}(\Omega)$ such that :

$$
\left\{\begin{array}{l}
-\Delta \varphi=\lambda \varphi \text { in } \Omega \\
\frac{\partial \varphi}{\partial y}=0 \text { on } \Gamma_{B} \\
\frac{\partial \varphi}{\partial y}=\omega^{2} \varphi \text { on } \Gamma_{F}
\end{array}\right.
$$

Then:

$$
\varphi(x, y)=\sum_{n=N(\lambda, \omega)}^{+\infty} a_{n}(\varphi) e^{-\alpha_{n}(x-d)} u_{n}(\omega ; y), \quad \forall(x, y) \in \Omega_{e}
$$

where : 
- $a_{n}(\varphi)=\int_{-h_{\infty}}^{0} \varphi(d, y) u_{n}(\omega ; y) d y$

- $N(\lambda, \omega)=\operatorname{Min}\left\{n \in \mathbf{N} ; \xi_{n}(\omega)>\lambda\right\}$

- $\alpha_{n}=\alpha_{n}(\lambda, \omega)=\left(\xi_{n}(\omega)-\lambda\right)^{1 / 2}$

- $\left(u_{n}(\omega), \xi_{n}(\omega)\right)_{n \geq 0}$ are the eigenelements of the operator $\hat{A}\left(\omega, h_{\infty}\right)$ defined above.

Proof : Let us consider the following function :

$$
v_{n}(x)=\int_{-h_{\infty}}^{0} \varphi(x, y) u_{n}(\omega ; y) d y, \text { for } x \geq d,
$$

which is $C^{\infty}$, since $\varphi \in C^{\infty}\left(\bar{\Omega}_{e}\right)$. Using the equations satisfied by $\varphi$ and by $u_{n}$, we obtain :

$$
\begin{aligned}
\frac{d^{2} v_{n}}{d x^{2}}(x) & =\int_{-h_{\infty}}^{0}\left(-\frac{\partial^{2} \varphi}{\partial y^{2}}(x, y)-\lambda \varphi(x, y)\right) u_{n}(\omega ; y) d y \\
& =\left(\xi_{n}(\omega)-\lambda\right) v_{n}(x)
\end{aligned}
$$

Moreover, $v_{n}$ is square integrable since :

$$
\left\|v_{n}\right\|_{L^{2}(d,+\infty)}^{2} \leq\|\varphi\|_{L^{2}\left(\Omega_{e}\right)}^{2}
$$

Consequently, we have :

- $v_{n}(x)=0, \forall x \geq d$, if $\xi_{n}(\omega)-\lambda \leq 0$

- $v_{n}(x)=v_{n}(d) e^{-\sqrt{\xi_{n}(\omega)-\lambda}(x-d)}$ if $\xi_{n}(\omega)-\lambda>0$.

The expression (3.9) follows, by the completeness of the family $\left(u_{n}\right)$, for every given value of $x \geq d$. Moreover, it is clear that the series and all its derivatives converge uniformly.

By the previous lemma, we see that a solution $\varphi$ of (3.8) is completely ans explicitly determined in the exterior domain $\Omega_{e}$ by the sequence of coefficients $a_{n}$ which depend only on the trace of $\varphi$ on the artificial boundary $\Sigma=\bar{\Omega}_{i} \cap \bar{\Omega}_{e}$.

Consider now the following problem, set in the bounded interior domain $\Omega_{i}$ :

$$
\left\{\begin{array}{l}
-\Delta \tilde{\varphi}=\lambda \tilde{\varphi} \text { in } \Omega_{i} \\
\frac{\partial \tilde{\varphi}}{\partial n}=0 \text { on } \Gamma_{B}^{i}=\Gamma_{B} \cap \Omega_{i} \\
\frac{\partial \tilde{\varphi}}{\partial y}=\omega^{2} \tilde{\varphi} \text { on } \Gamma_{F}^{i}=\Gamma_{F} \cap \Omega_{i} \\
\frac{\partial \tilde{\varphi}}{\partial x}=-T(\omega, \lambda)\left(\left.\tilde{\varphi}\right|_{\Sigma}\right) \text { on } \Sigma
\end{array}\right.
$$

where the operator $T(\omega, \lambda)$ is defined (with the notations of lemma 3.2) by :

$$
T(\omega, \lambda)\left\{\begin{array}{l}
H^{1 / 2}(\Sigma) \longmapsto H^{-1 / 2}(\Sigma) \\
u=\sum_{n=0}^{+\infty} a_{n} u_{n}(\omega) \longmapsto \sum_{n=N(\lambda, \omega)}^{+\infty} \alpha_{n}(\lambda, \omega) a_{n} u_{n}(\omega)
\end{array}\right.
$$


Notice that $T$ is continuous since, with the notation (3.6):

$$
\begin{aligned}
\|T u\|_{-1 / 2}^{2} & =\sum_{n=N(\lambda, \omega)}^{+\infty} \nu_{n}^{-1}(\omega) a_{n}^{2} \alpha_{n}^{2}(\lambda, \omega) \\
& \leq\left(1+\frac{\lambda}{\min _{n \geq N(\lambda, \omega)}\left|\xi_{n}(\omega)\right|}\right) \sum_{n=0}^{+\infty} \nu_{n}(\omega) a_{n}^{2} \\
& \leq\left(1+\frac{\lambda}{\min _{n \geq 0}\left|\xi_{n}(\omega)\right|}\right)\|u\|_{1 / 2}^{2} .
\end{aligned}
$$

Then we have the

Proposition 3.1 If $\varphi \in H^{1}(\Omega)$ is a solution of (3.8), then $\varphi \mid \Omega_{i}$ is a solution of (3.10). Conversely, if $\dot{\varphi} \in H^{1}\left(\Omega_{i}\right)$ is a solution of (3.10) and if :

$$
\int_{-h_{\infty}}^{0} \tilde{\varphi}(d, y) u_{n}(\omega ; y) d y=0 \text { for } n<N(\lambda, \omega)
$$

then $\varphi$ is a solution of (3.8), if we set :

$$
\left\{\begin{array}{l}
\text { For } x<d: \varphi(x, y)=\tilde{\varphi}(x, y) \\
\text { For } x>d: \varphi(x, y)=\sum_{n=N(\lambda, \omega)}^{+\infty} a_{n} e^{-\alpha_{n}(\lambda, \omega)(x-d)} u_{n}(y)
\end{array}\right.
$$

with $a_{n}=\int_{-h_{\infty}}^{0} \tilde{\varphi}(d, y) u_{n}(\omega ; y) d y$ and with $N(\lambda, \omega), \alpha_{n}(\lambda, \omega)$ and $u_{n}(\omega)$ defined as in lemma 3.2.

Proof: The first part of the proposition is a direct consequence of lemma 3.2. Conversely, notice that the function $\varphi$ defined by (3.12) is such that $\varphi$ and $\frac{\partial \varphi}{\partial x}$ are continuous through $\Sigma$, so that the partial differential equation

$$
-\Delta \varphi=\lambda \varphi
$$

is satisfied in $\Omega$. Moreover, one can verify that $\varphi \in H^{1}(\Omega)$ as soon as :

$$
\sum a_{n}^{2} \alpha_{n}(\lambda, \omega)<+\infty
$$

which holds since $\left.\bar{\varphi}\right|_{\Sigma} \in H^{1 / 2}(\Sigma)$.

Remark 3.2 If $\lambda<-\beta_{\infty}^{2}(\omega)$, problems (3.8) and (3.10) are equivalent, since $N(\lambda, \omega)=$ 0 . 
Let us consider the unbounded operator of $L^{2}\left(\Omega_{i}\right), B(\omega, \lambda)$, defined by :

$$
\left\{\begin{aligned}
& D(B(\omega, \lambda))=\left\{\varphi \in L^{2}\left(\Omega_{i}\right) ; \Delta \varphi \in L^{2}\left(\Omega_{i}\right), \frac{\partial \varphi}{\partial n}=0 \text { on } \Gamma_{B}^{i}\right. \\
&\left.\frac{\partial \varphi}{\partial n}=\omega^{2} \varphi \text { on } \Gamma_{F}^{i}, \frac{\partial \varphi}{\partial n}=-T(\omega, \lambda) \varphi \text { on } \Sigma\right\} \\
& B(\omega, \lambda) \varphi=-\Delta \varphi \text { for } \varphi \in D(B(\omega, \lambda))
\end{aligned}\right.
$$

Then, every solution $(\varphi, \lambda)$ of $(3.8)$ is such that

$$
\left\{\begin{array}{l}
\varphi \in D(B(\omega, \lambda)), \varphi \neq 0 \\
B(\omega, \lambda) \varphi=\lambda \varphi
\end{array}\right.
$$

In particular, (3.14) is equivalent to (3.8) if $\lambda<-\beta_{\infty}^{2}(\omega)$.

Formulation (3.4) is linear with respect to $\beta^{2}$ but it is set in an unbounded domain, while formulation (3.14), set in the bounded domain $\Omega_{i}$, is non-linear. 


\section{The basic spectral theory of the operator $A(\omega)$}

In this section, we give a qualitative description of the spectrum of $A(\omega)$. In particular, we derive a lower bound for the spectrum and we determine the essential spectrum. The eigenvalues located below the essential spectrum are characterized by the Min-Max principle and the question of possible eigenvalues embedded in the essential spectrum is discussed.

\subsection{Selfadjointness of $A(\omega)$ and lower bound for the spectrum}

Let $a(\omega ; \cdot, \cdot)$ be the symmetric continuous bilinear form in $H^{1}(\Omega)$ defined by :

$$
a(\omega ; \varphi, \psi)=\int_{\Omega} \nabla \varphi \cdot \nabla \psi d x d y-\omega^{2} \int_{\Gamma_{F}} \varphi \psi d \sigma .
$$

Thanks to the Green's formula, we see that :

$$
(A(\omega) \varphi, \psi)=a(\omega ; \varphi, \psi), \quad \forall(\varphi, \psi) \in H^{1}(\Omega)^{2},
$$

where $(\cdot, \cdot)$ denotes the usual inner product of $L^{2}(\Omega)$ and where $A(\omega)$ is defined by (3.1) and $(3.2)$.

This proves that $A(\omega)$ is symmetric and that $H^{1}(\Omega)$ is the form-domain of $A(\omega)$. The main result of this section is the following :

Theorem 4.1 The operator $A(\omega)$ is self-adjoint

Proof: As $A(\omega)$ is symmetric, it suffices to show that $A(\omega)+\lambda I$ is surjective for some real $\lambda$. Thanks to the Lax-Milgram lemma, this is a direct consequence of the following lemma :

Lemma 4.1 There exists a constant $\Lambda(\omega)>0$ such that the bilinear form $a(\omega ; \varphi, \psi)+$ $\lambda(\varphi, \psi)$ is coercive in $H^{1}(\Omega)$ as soon as $\lambda>\Lambda(\omega)$.

Proof:For any $\varphi \in H^{1}(\Omega)$, we can write :

$$
a(\omega ; \varphi, \varphi)=\int_{\Omega_{e}}|\nabla \varphi|^{2} d x d y-\omega^{2} \int_{\Gamma_{F}^{e}}^{i}|\varphi|^{2} d \sigma+\int_{\Omega_{i}}|\nabla \varphi|^{2} d x d y-\omega^{2} \int_{\Gamma_{F}^{i}}|\varphi|^{2} d \sigma
$$

where $\Omega_{i}$ and $\Omega_{e}$ are the subdomains introduced in section 3.2 and $\Gamma_{F}^{i}$ and $\Gamma_{F}^{e}$ the corresponding free surfaces. Now, let us write for any $0<\epsilon<1$ :

$$
\begin{aligned}
& (1-\epsilon) \int_{\Omega_{e}}|\nabla \varphi|^{2} d x d y-\omega^{2} \int_{\Gamma_{F}^{e}}|\varphi|^{2} d \sigma= \\
& \quad(1-\epsilon) \int_{\Omega_{e}}\left|\frac{\partial \varphi}{\partial x}\right|^{2} d x d y+(1-\epsilon) \int_{d}^{+\infty}\left(\int_{-h_{\infty}}^{0}\left|\frac{\partial \varphi}{\partial y}(x, y)\right|^{2} d y-\frac{\omega^{2}}{(1-\epsilon)}|\varphi(x, 0)|^{2}\right) d x
\end{aligned}
$$

Setting $\beta_{\infty}^{\epsilon}(\omega)=\beta_{\infty}(\omega / \sqrt{1-\epsilon})$ where $\beta_{\infty}$ is defined by $(2.2)$, we deduce by integrating (3.7) :

$$
\int_{\Omega_{e}}|\nabla \varphi|^{2} d x d y-\frac{\omega^{2}}{(1-\epsilon)} \int_{\Gamma_{F}^{e}}|\varphi|^{2} d \sigma \geq \int_{\Omega_{e}}\left|\frac{\partial \varphi}{\partial x}\right|^{2} d x d y-\beta_{\infty}^{\epsilon}(\omega)^{2} \int_{\Omega_{e}}|\varphi|^{2} d x d y
$$


Consequently, we get :

$$
\begin{aligned}
& \int_{\Omega_{e}}|\nabla \varphi|^{2} d x d y-\omega^{2} \int_{\Gamma_{F}^{e}}|\varphi|^{2} d \sigma \geq \\
& \quad \geq \epsilon \int_{\Omega_{e}}|\nabla \varphi|^{2} d x d y-(1-\epsilon) \beta_{\infty}^{\epsilon}(\omega)^{2} \int_{\Omega_{e}}|\varphi|^{2} d x d y
\end{aligned}
$$

Let us admit for a while the following lemma

Lemma 4.2 For any $\eta>0$, there exists $C(\eta)>0$ such that :

$$
\forall \phi \in H^{1}\left(\Omega_{i}\right), \quad \int_{\Gamma_{F}^{i}}|\varphi|^{2} d \sigma \leq \eta \int_{\Omega_{i}}|\nabla \varphi|^{2} d x d y+C(\eta) \int_{\Omega_{i}}\left|\varphi^{2}\right| d x d y .
$$

Thanks to lemma 4.2 , we can write

$$
\begin{aligned}
\int_{\Omega_{1}}|\nabla \phi|^{2} d x d y-\omega^{2} \int_{\Gamma_{F}^{2}}|\varphi|^{2} d x & \geq\left(1-\eta \omega^{2}\right) \int_{\Omega_{i}}|\nabla \varphi|^{2} d x d y \\
& -C(\eta) \omega^{2} \int_{\Omega_{1}}|\varphi|^{2} d x d y
\end{aligned}
$$

Let us choose $\eta$ such that $1-\eta \omega^{2}=\epsilon$, so that we can regroup (4.3) and (4.4) to obtain :

$$
\begin{aligned}
\int_{\Omega}|\nabla \phi|^{2} d x d y-\omega^{2} \int_{\Gamma_{F}}|\varphi|^{2} d \sigma & \geq \epsilon \int_{\Omega}|\nabla \varphi|^{2} d x d y \\
& -\max \left((1-\epsilon) \beta_{\infty}^{\epsilon}(\omega)^{2}, C(\eta) \omega^{2}\right) \int_{\Omega}|\varphi|^{2} d x d y
\end{aligned}
$$

This proves that $a(\omega ; \varphi, \varphi)+\lambda(\varphi, \varphi)$ is coercive in $H^{1}(\Omega)$ as soon as :

$$
\lambda>\max \left((1-\epsilon) \beta_{\infty}^{\epsilon}(\omega)^{2}, C(\eta) \omega^{2}\right)
$$

Proof of Lemma 4.2: It the lemma was false, there would exist a constant $C>0$ and a sequence $\varphi_{n}$ in $H^{1}\left(\Omega_{i}\right)$ such that :

$$
\mid \begin{aligned}
& \int_{\Gamma^{i}{ }^{*}}\left|\varphi_{n_{i}}^{2}\right| d \sigma=1 \\
& \int_{\Omega_{i}}\left|\varphi_{n}^{2}\right| d x d y \leq \frac{1}{n} \\
& \int_{\Omega_{1}}\left|\nabla \varphi_{n}^{2}\right| d x d y \leq C
\end{aligned}
$$

Thanks to the trace theorem we deduce that $\left.\varphi_{n}\right|_{\Gamma_{F}^{i}}$ is bounded in $H^{1 / 2}\left(\Gamma_{F}^{i}\right)=H^{1 / 2}([0, d])$. As $H^{1 / 2}\left(\Gamma_{F}^{i}\right)$ is compactly embedded into $L^{2}\left(\Gamma_{F}^{i}\right)$, we can extract from $\varphi_{n}$ a subsequence, still denoted by $\varphi_{n}$, such that

$$
\mid \begin{array}{lll}
\varphi_{n} \rightarrow 0 & \text { in } L^{2}\left(\Omega_{i}\right) & \text { strongly } \\
\varphi_{n} \rightarrow 0 & \text { in } H^{1}\left(\Omega_{i}\right) & \text { weakly } \\
\left.\varphi_{n}\right|_{\Gamma_{F}^{i}} \rightarrow \psi & \text { in } L^{2}\left(\Gamma_{F}^{i}\right) & \text { strongly }
\end{array}
$$


By weak convergence of $\varphi_{n}$ in $H^{1}\left(\Omega_{i}\right)$, we deduce that $\psi \doteq 0$ which is impossible since by strong convergence in $L^{2}\left(\Gamma_{F}^{i}\right)$ we have $\int_{\Gamma_{F}^{i}}|\psi|^{2} d \sigma=1$

Remark 4.1 Note that the result does not suppose any smoothness of the boundary $\partial \Omega$ since we use only the compactness of the canonical injection from $H^{1 / 2}\left(\Gamma_{F}^{i}\right)$ into $L^{2}\left(\Gamma_{F}^{i}\right)$.

Now, let $b(\omega, \lambda ; \varphi, v)$ be the symmetric continuous bilinear form in $H^{1}\left(\Omega_{i}\right)$ associated to the operator $B(\omega, \lambda)$ defined by $(3.13)$ :

$$
\left.b(\omega, \lambda ; \varphi, \psi)=\int_{\Omega_{i}} \nabla \varphi \nabla \psi d x d y-\omega^{2} \int_{\Gamma_{F}^{\prime}} \varphi \psi d \sigma+<T(\omega, \lambda) \varphi, \psi\right\rangle_{\Sigma}
$$

Here, $<.,.\rangle_{\Sigma}$ denotes the duality product between $H^{-1 / 2}(\Sigma)$ and $H^{1 / 2}(\Sigma)$.

Now, let us state for the operator $B(\omega, \lambda)$ a result which is equivalent to theorem 4.1 for the operator $A(\omega)$.

Theorem 4.2 The bilinear form $b(\omega, \lambda ; \varphi, \psi)+\mu(\varphi, \psi)$ is coercive in $H^{1}\left(\Omega_{i}\right)$ as soon as $\mu>\Lambda(\omega)$ where $\Lambda(\omega)$ is the constant defined in lemma 4.1. Consequently, the operator $B(\omega, \lambda)$ is sclf-adjoint.

Proof :

The proof is straightforward, if we notice that :

$$
<T(\omega, \lambda) \varphi, \varphi>_{\Sigma}=\sum_{n=N(\lambda, \omega)}^{+\infty} \alpha_{n} a_{n}^{2} \geq 0, \quad \forall \varphi \in H^{1}\left(\Omega_{i}\right)
$$

(where $N(\lambda, \omega), \alpha_{n}$ and $a_{n}$ are defined in lemma 3.2).

Let us denote by $\sigma(\omega)$ the spectrum of $A(\omega)$ and by $\sigma(\omega, \lambda)$ the spectrum of $B(\omega, \lambda)$. As a direct consequence of theorems 4.1 and 4.2 , we have the following inclusions.

$$
\left\{\begin{array}{l}
\sigma(\omega) \subset[-\Lambda(\omega),+\infty] \\
\sigma(\omega, \lambda) \subset[-\Lambda(\omega),+\infty]
\end{array}\right.
$$

In other words, $\Lambda(\omega)$ is a lower bound for the spectra of $A(\omega)$ and $B(\omega, \lambda)$. Since lemma 4.2 has been established by contradiction, we have generally no numerical estimates for $\Lambda(\omega)$. However, for some particular depth profiles, we can derive directly an explicit expression of a lower bound for $\sigma(\omega)$ and $\sigma(\omega, \lambda)$. Indeed, we have the

Lemma 4.3 (i) If $h^{-}>0$, where $h^{-}$is defined by (2.1), then we can choose in (4.6) $\Lambda(\omega)=\left(\beta^{-}(\omega)\right)^{2}$ where $\beta^{-}(\omega)$ is the positive solution $\beta$ of :

$$
\beta \tanh \left(\beta h^{-}\right)=\omega^{2}
$$


(ii) If the function $h(x)$ satisfies for some $\alpha \in] 0, \frac{\pi}{2}[$ :

$$
h(x) \geq \operatorname{Min}\left(x \tan \alpha, h_{\infty}\right)
$$

then we can choose in $(4.0) \quad \Lambda(\omega)=\sin ^{2} \alpha \beta_{\infty}^{2}\left(\frac{\omega}{\sin \alpha}\right)$ where the function $\beta_{\infty}$ is defined by (2.2).

These two situations are illustrated by figure 7

\section{Proof :}

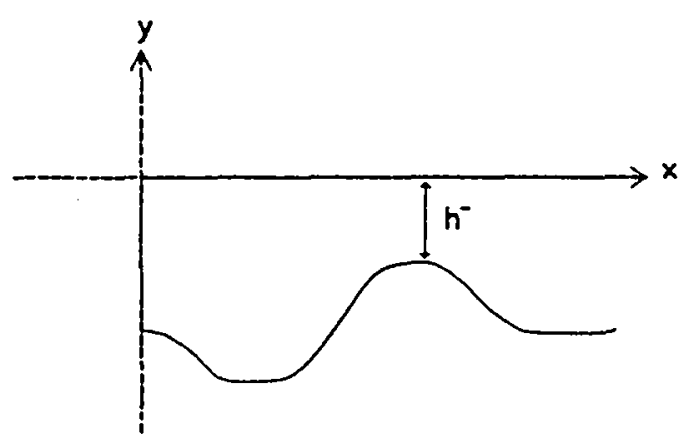

Figure 7: (i) $h->0$

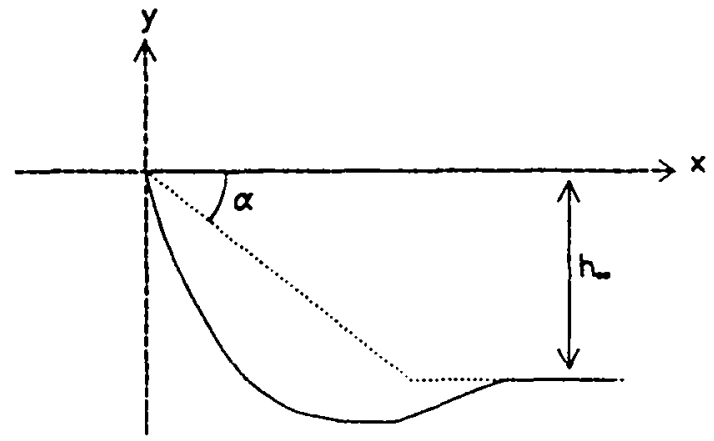

(ii) $h(x) \geq \operatorname{Min}\left(x \tan \alpha, h_{\infty}\right)$

(i) By (3.7), we have

$$
\int_{0}^{+\infty} \int_{-h^{-}}^{0}\left|\frac{\partial \varphi}{\partial x}\right|^{2} d x d y+\beta^{-}(\omega)^{2} \int_{0}^{+\infty} \int_{-h^{-}}^{0} \varphi^{2} d x d y \geq \omega^{2} \int_{0}^{+\infty} \varphi^{2}(x, 0) d x
$$

for every $\varphi \in H^{1}(] 0,+\infty[\times]-h^{-}, 0[)$. Assertion (i) follows since, by definition of $h^{-}$:

$$
] 0,+\infty[x]-h^{-}, 0[\subset \Omega
$$

(ii) Set

$$
\left\{\begin{array}{l}
X=x+\cot \alpha y, Y=y \\
\varphi(x, y)=\psi(X, Y)
\end{array}\right.
$$

Then by (4.7) we have for every $\varphi$ in $H^{1}(\Omega)$

$$
a(\omega ; \varphi, \varphi) \geq \int_{0}^{+\infty} \int_{-h_{\infty}}^{0}\left\{\left|\frac{\partial \psi}{\partial X}\right|^{2}+\left|\frac{\partial \psi}{\partial Y}+\cot \alpha \frac{\partial \psi}{\partial X}\right|^{2}\right\} d X d Y-\omega^{2} \int_{0}^{+\infty} \psi^{2}(X, 0) d X .
$$


so that, for every $c>0$ :

$$
\begin{aligned}
a(\omega ; \varphi ; \varphi) & \geq \int_{0}^{+\infty} \int_{-\iota_{\infty}}^{0}\left\{\left(1+\cot ^{2} \alpha-\epsilon\right)\left|\frac{\partial \psi}{\partial X}\right|^{2}+\left(1-\frac{\cot ^{2} \alpha}{\epsilon}\right)\left|\frac{\partial \psi}{\partial Y}\right|^{2} d X d Y\right. \\
& -\omega^{2} \int_{0}^{+\infty} \psi^{2}(X, 0) d X
\end{aligned}
$$

Assertion (ii) follows then from (3.7) by taking :

$$
\epsilon=1+\cot ^{2} \alpha
$$

\subsection{The essential spectrum}

Our aim is now to determine the essential spectrum $\sigma_{e s s}(\omega)$ of the operator $A(\omega)$.

First we prove the

Theorem 4.3 The following embedding holds:

$$
\left[-\beta_{\infty}^{2}(\omega),+\infty\left[\subset \sigma_{e s s}(\omega)\right.\right.
$$

where $\beta_{\infty}(\omega)$ is defined by (2.2).

Proof : Since $\sigma_{e s s}(\omega)$ is a closed set, it is sufficient to prove the following inclusion:

$$
]-\beta_{\infty}^{2}(\omega),+\infty\left[\subset \sigma_{e s s}(\omega)\right.
$$

Let $\sigma<\beta_{\infty}^{2}(\omega)$ and consider the sequence of functions

$$
\psi_{n}(x, y)=\theta_{n}(x) \psi(x, y) \quad n \in \mathrm{N}
$$

with

$$
\left\{\begin{array}{l}
\psi(x, y)=\cosh \left(\beta_{\infty}(\omega)\left(y+h_{\infty}\right)\right) \exp \left(i\left(\beta_{\infty}^{2}(\omega)-\sigma\right)^{1 / 2} x\right) \\
\theta_{n}(x)=\frac{1}{\sqrt{n}} \theta\left(\frac{x-x_{0}-1}{n}\right)
\end{array}\right.
$$

where $x_{0}$ is such that $h(x)=h_{\infty}$ for $x>x_{0}$ and where $\theta$ is a $C_{\infty}$ function such that :

$$
\left\{\begin{array}{lll}
0 \leq \theta(x) \leq 1 & \forall x \in \mathbf{R} \\
\theta(x)=0 & \text { if } \quad x \leq-1 \text { or } x \geq 2 \\
\theta(x)=1 & \text { if } \quad 0 \leq x \leq 1
\end{array}\right.
$$

To prove that $(-\sigma) \in \sigma_{e s s}(\omega)$, it suffices to show that $\left(\psi_{n}\right)_{n}$ is a singular sequence i.e. : 


$$
\begin{aligned}
& \text { (i) } \liminf _{n>0}\left|\psi_{n}\right|{ }_{\Omega}>0 \\
& \text { (ii) } \psi_{n} \in D(A(\omega)) \text { and } \lim _{n \rightarrow+\infty}\left|A(\omega) \psi_{n}+\sigma \psi_{n}\right|_{\Omega 2}=0
\end{aligned}
$$

First we have :

$$
\int_{\Omega}\left|\psi_{n}\right|^{2} d x d y=g(\omega) \int_{\mathbb{R}} 0(x)^{2} d x
$$

where:

$$
g(\omega)=\int_{-h_{\infty}}^{0} \cosh ^{2}\left(\beta_{\infty}(\omega)\left(y+h_{\alpha^{\prime}}\right)\right) d y
$$

That proves (i).

Moreover, it is easy to verify that $\psi_{n} \in D(A(\omega))$, and we have :

$$
\begin{aligned}
\Delta \psi_{n} & =\theta_{n} \Delta \psi+2 \frac{\partial \psi}{\partial x} \theta_{n}^{\prime}+\psi \theta_{n}^{\prime \prime} \\
& =\sigma \psi_{n}+2 i\left(\beta_{\infty}^{2}(\omega)-\sigma\right)^{1 / 2} \psi 0_{n}^{\prime}+\psi \theta_{n}^{\prime \prime}
\end{aligned}
$$

Consequently :

$$
\left|A(\omega) \psi_{n}+\sigma \psi_{n}\right|_{2}^{2} \leq g(\omega)\left(2\left(\beta_{\infty}^{2}(\omega)-\sigma\right)^{1 / 2} \int_{\mathbf{R}} \theta_{n}^{\prime}(x)^{2} d x+\int_{\mathbb{R}} \theta_{n}^{\prime \prime}(x)^{2} d x\right)
$$

But :

which proves (ii).

$$
\left\{\begin{array}{l}
\int_{\mathbb{R}} \theta_{n}^{\prime}(x)^{2} d x=\frac{1}{n^{2}} \int_{\mathbf{R}} \theta^{\prime}(x)^{2} d x \\
\int_{\mathbb{R}} \theta_{n}^{\prime \prime}(x)^{2} d x=\frac{1}{n^{4}} \int_{\mathbf{R}} \theta^{\prime \prime}(x)^{2} d x
\end{array}\right.
$$

Let us consider now the following bilinear forms :

$$
\begin{aligned}
& d(\omega ; \varphi, \psi)= \int_{\Omega} \frac{\partial \varphi}{\partial y} \frac{\partial \psi}{\partial y} d x d y+\beta_{\infty}^{2}(\omega) \int_{\Omega} \varphi \psi d x d y \\
&-\beta_{\infty}(\omega) \int_{0}^{+\infty} \tanh \left(\beta_{\infty}(\omega) h(x)\right) \varphi(x, 0) \psi(x, 0) d x \\
& p(\omega ; \varphi, \psi)=\int_{0}^{+\infty}\left\{\beta_{\infty}(\omega) \tanh \left(\beta_{\infty}(\omega) h(x)\right)-\omega^{2}\right\} \varphi(x, 0) \psi(x, 0) d x
\end{aligned}
$$

Then we have the :

Lemma 4.4 (i) The bilinear form $a(\omega ; \varphi, \psi)$, defined by (4.1), can be written as :

$$
\begin{aligned}
a(\omega ; \varphi, \psi) & =-\beta_{\infty}^{2}(\omega) \int_{\Omega} \varphi \psi d x d y+\int_{\Omega} \frac{\partial \varphi}{\partial x} \frac{\partial \psi}{\partial x} d x d y \\
& +d(\omega ; \varphi, \psi)+p(\omega ; \varphi, \psi)
\end{aligned}
$$


(ii) The bilinear form $d(\omega ; .,$.$) is positive$

(iii) The bilinear form $p(\omega ; .,$.$) is compact in H^{1}(\Omega)$ i.e.

$$
\varphi_{n} \rightarrow \varphi \text { weakly in } H^{1}(\Omega) \Longrightarrow p\left(\omega ; \varphi_{n}, \varphi_{n}\right) \longrightarrow p(\omega ; \varphi, \varphi)
$$

Proof: The decomposition (4.10) is obvious. The assertion (ii) is a consequence of (3.7). The assertion (iii) results from the compacity of the trace application :

$$
\varphi \in H^{1}(\Omega) \longmapsto \varphi(x, 0) \in L^{2}\left(\left[0, x_{0}\right]\right) .
$$

Using the previous lemma, we can prove the

Theorem 4.4 One has the following equality:

$$
\sigma_{e s s}(\omega)=\left[-\beta_{\infty}^{2}(\omega),+\infty\right]
$$

where $\beta_{\infty}(\omega)$ is defined by (2.2).

Proof : By theorem 4.3, it remains to establish the following inclusion :

$$
\sigma_{e s s}(\omega) \subset\left[-\beta_{\infty}^{2}(\omega),+\infty[\right.
$$

Let $\lambda \in \sigma_{\epsilon s s}(\omega)$. Then there exists a sequence $\left(\psi_{n}\right)_{n \in \mathbb{N}}$ of $D(A(\omega))$ such that :

$$
\begin{aligned}
& \left|\psi_{n}\right|_{\Omega}=1 \quad \forall n \in \mathrm{N} \\
& A(\omega) \psi_{n}-\lambda \psi_{n} \rightarrow 0 \text { strongly in } L^{2}(\Omega) \\
& \psi_{n} \rightarrow 0 \text { weakly in } L^{2}(\Omega)
\end{aligned}
$$

By (4.11), $a\left(\omega ; \psi_{n}, \psi_{n}\right)$ tends to $\lambda$ as $n$ tends to infinity. Consequently, by (4.10):

$$
\left|\frac{\partial \dot{\psi}_{n}}{\partial x}\right|_{\Omega}^{2}+d\left(\omega ; \psi_{n}, \psi_{n}\right)+p\left(\omega ; \psi_{n}, \psi_{n}\right) \longrightarrow \lambda+\beta_{\infty}^{2} \dot{(\omega)}
$$

But by lemma 4.4 and by (4.11):

$$
p\left(\omega ; \psi_{n}, \psi_{n}\right) \longrightarrow 0
$$

It results then from the positivity of $d(\omega ; \psi, \psi)$ that :

$$
\lambda+\beta_{\infty}^{2}(\omega) \geq 0
$$

and that achieves the proof. 


\subsection{On the existence of eigenvalues in the essential spectrum}

A difficult question concerns the existence of eigenvalues $\lambda$ of the operator $A(\omega)$ such that

$$
\lambda \geq-\beta_{\infty}^{2}(\omega) .
$$

In, fact this problem is still open. It is related to the very difficult problem of uniqueness for the linearized two-dimensional water-wave problem (cf.[12], [24]). The study of the existence of embedded eienvalues leads, in other applications, to different conclusions depending on the problem which is considered. The absence of embedded eigenvalues has been proved in particular for Schroedinger operators ( $\mathrm{cf}[23]$ ) and for the acoustic propagator in stratified media ( $\mathrm{cf}[32]$ ). However, examples of embedded eigenvalues have been exhibited in various cases ( $\mathrm{cf}[6],[13],[35])$. For our problem we give a partial solution by proving the :

Theorem 4.5 The eigenvalues of $A(\omega)$ have finite multiplicity and form, at most, a countable sequence tending to infinity.

As a consequence of theorem 4.5 and of (4.6), we have the

Corollary 4.1 The operator $A(\omega)$ has at most a finite number of eigenvalues below the essential spectrum.

Remark 4.2 Corollary 4.1 can be obtained by various other techniques (cf. [14],[31]) and corollary 5.3. For the purpose of this section, theorem 4.5 proves moreover that there are at most a countable set of eigenvalues embedded in the essential spectrum.

Proof of theorem 4.5: To establish this result, we will proceed as Nedelec and Starling (cf. [21]). By the proposition 3.1, it is sufficient to prove that the values $\lambda \in \mathbb{R}$ such that $\lambda$ is an eigenvalue of $B(\omega, \lambda)$ form a sequence $\lambda_{n}$ such that $\lambda_{n} \rightarrow+\infty$.

By theorem 4.2.' and by Lax-Milgram's theorem, the operator $B(\omega, \lambda)+\mu I$ is invertible for $\mu>\Lambda(\omega)$. The operator $(B(\omega, \lambda)+\mu I)^{-1}$ is continous from $L^{2}\left(\Omega_{i}\right)$ into $H^{1}\left(\Omega_{i}\right)$ and since $\Omega_{i}$ is bounded, $(B(\omega, \lambda)+\mu I)^{-1}$ is compact from $L^{2}\left(\Omega_{i}\right)$ into $L^{2}\left(\Omega_{i}\right)$. From the classical results of spectral theory for compact self-adjoint operators, we deduce therefore the

Lemma 4.5 The spectrum $\sigma(\omega, \lambda)$ of $B(\omega, \lambda)$ consists in an increasing sequence of real eigenvalues $\left(\mu_{n}(\omega, \lambda)\right)_{n \geq 1}$ such that $\mu_{n}(\omega, \lambda) \rightarrow+\infty$. Moreover, $\mu_{n}(\omega, \lambda)$ is charactcrized by the following formula :

$$
\text { (4.14) } \quad \mu_{n}(\omega, \lambda)=\min _{V \in \mathcal{V}_{n}\left(\Omega_{1}\right)} \max _{\varphi \in V, \varphi \neq 0} \frac{b(\omega, \lambda ; \varphi, \varphi)}{|\varphi|_{\Omega_{i}}^{2}}
$$

where $\mathcal{V}_{n}\left(\Omega_{i}\right)$ denotes the set of all $n$-dimensional subspaces of $H^{1}\left(\Omega_{i}\right)$. (Each eigenvalue is repeated a number of times equal to its order of multiplicity). 
Consequently, a value $\lambda \in \mathbb{R}$ is an eigenvalue of $B(\omega, \lambda)$ if and only if $\lambda$ satisfies for some $n \geq 1$ :

$$
\mu_{n}(\omega, \lambda)=\lambda .
$$

Let us admit for a while the following result :

Lemma 4.6 The function $\lambda \longmapsto \mu_{n}(\omega, \lambda)$ is continuous and non-increasing in $\lambda$.

Then, it is obvious that equation (4.14) has a unique solution $\lambda_{n}$. Moreover, the sequence $\lambda_{n}$ tends to infinity whith $n$. Indeed, suppose that $\lambda_{n} \leq \lambda$ for every $n$. Then :

$$
\mu_{n}(\omega, \lambda) \leq \mu_{n}\left(\omega, \lambda_{n}\right)=\lambda_{n} \leq \lambda, \quad \forall n \geq 1
$$

and that is impossible since $\mu_{n}(\omega, \lambda) \rightarrow+\infty$.

That achieves the proof of theorem 4.5 .

\section{Proof of lemma 4.6:}

One can check easily that, for any given $\varphi \in H^{1}\left(\Omega_{i}\right)$, the function $\lambda \longmapsto \frac{b(\omega, \lambda ; \varphi, \varphi)}{|\varphi|_{\Omega_{1}}^{2}}$ is non-increasing in $\lambda$. The same is a fortiori true for $\lambda \longmapsto \mu_{n}(\omega, \lambda)$. It remains to establish the continuity of $\lambda \longmapsto \mu_{n}(\omega, \lambda)$. Let $\lambda<\lambda^{\prime}$ and $\varphi \in H^{1}\left(\Omega_{i}\right)$. Then :

$$
b(\omega, \lambda ; \varphi, \varphi)-b\left(\omega, \lambda^{\prime} ; \varphi, \varphi\right)=<\left(T(\omega, \lambda)-T\left(\omega, \lambda^{\prime}\right)\right) \varphi, \varphi>_{\Sigma} .
$$

In the following, we use the notations of lemma 3.2. We consider two cases :

Case 1: $\xi_{m-1} \leq \lambda \leq \lambda^{\prime}<\xi_{m}$ for some $m>1$

Then :

$$
N(\lambda)=N\left(\lambda^{\prime}\right):=m
$$

and therefore :

$$
\begin{aligned}
b(\omega, \lambda ; \varphi, \varphi)-b\left(\omega, \lambda^{\prime} ; \varphi, \varphi\right) & =\sum_{n=m}^{+\infty}\left(\sqrt{\xi_{n}-\lambda}-\sqrt{\xi_{n}-\lambda^{\prime}}\right) a_{n}^{2} \\
& \leq\left(\lambda^{\prime}-\lambda\right) \sum_{n=m}^{+\infty} \frac{a_{n}^{2}}{\sqrt{\xi_{n}-\lambda}+\sqrt{\xi_{n}-\lambda^{\prime}}} \\
& \leq\left(\lambda^{\prime}-\lambda\right)\left(\sqrt{\xi_{n}-\lambda}+\sqrt{\xi_{n}-\lambda^{\prime}}\right)^{-1}\left\|\left.\varphi\right|_{\Sigma}\right\|_{L^{2}(\Sigma)}^{2}
\end{aligned}
$$

Case 2 : $\xi_{m-1} \leq \lambda \leq \lambda^{\prime}=\xi_{m}$ for some $m>1$

Then :

$$
N(\lambda)=m \text { and } N\left(\lambda^{\prime}\right)=m+1
$$


and therefore :

$$
\begin{aligned}
b(\omega, \lambda ; \varphi, \varphi)-b\left(\omega, \lambda^{\prime} ; \varphi, \varphi\right) & =\sqrt{\lambda^{\prime}-\lambda} a_{m}^{2}+\sum_{n=m+1}^{+\infty}\left(\sqrt{\xi_{n}-\lambda}-\sqrt{\xi_{n}-\lambda^{\prime}}\right) a_{n}^{2} \\
& \leq\left(\sqrt{\lambda^{\prime}-\lambda}+\frac{\lambda^{\prime}-\lambda}{2 \sqrt{\xi_{m+1}-\xi_{m}}}\right)\left\|\left.\varphi\right|_{\Sigma}\right\|_{L^{2}(\Sigma)}^{2}
\end{aligned}
$$

Consequently, we have proved that, for every $\varphi \in H^{1}\left(\Omega_{i}\right)$ and for $\lambda \leq \lambda^{\prime}$ :

$$
0 \leq b(\omega, \lambda ; \varphi, \varphi)-b\left(\omega, \lambda^{\prime} ; \varphi, \varphi\right) \leq \epsilon\left(\lambda, \lambda^{\prime}\right)\left\|\left.\varphi\right|_{\Sigma}\right\|_{L^{2}(\Sigma)}^{2}
$$

where $\epsilon\left(\lambda, \lambda^{\prime}\right)$ is independent on $\varphi$ and tends to 0 when $\left(\lambda^{\prime}-\lambda\right)$ tends to 0 .

Now, by theorem 4.2 and by the continuity of the trace application from $H^{1}\left(\Omega_{i}\right)$ into $L^{2}(\Sigma)$, there exist two constants $C(\omega)$ and $\mu>\Lambda(\omega)$ such that :

$$
\left\|\left.\varphi\right|_{\Sigma}\right\|_{L^{2}(\Sigma)}^{2} \leq C(\omega)\left(b\left(\omega, \lambda^{\prime} ; \varphi, \varphi\right)+\mu|\varphi|_{\Omega_{i}}^{2}\right), \quad \forall \varphi \in H^{1}\left(\Omega_{i}\right) .
$$

From (4.16) and (4.17) we deduce, for $\varphi \neq 0$ :

$$
\frac{b\left(\omega, \lambda^{\prime} ; \varphi, \varphi\right)}{|\varphi|_{\Omega_{i}}^{2}} \leq \frac{b(\omega, \lambda ; \varphi, \varphi)}{|\varphi|_{\Omega_{i}}^{2}} \leq \frac{b\left(\omega, \lambda^{\prime} ; \varphi, \varphi\right)}{|\varphi|_{\Omega_{1}}^{2}}\left(1+C(\omega) \epsilon\left(\lambda, \lambda^{\prime}\right)\right)+\mu C(\omega) \epsilon\left(\lambda, \lambda^{\prime}\right)
$$

and consequently

$$
\mu_{m}\left(\omega, \lambda^{\prime}\right) \leq \mu_{m}(\omega, \lambda) \leq \mu_{m}\left(\omega, \lambda^{\prime}\right)\left(1+C(\omega) \epsilon\left(\lambda, \lambda^{\prime}\right)\right)+\mu C(\omega) \epsilon\left(\lambda, \lambda^{\prime}\right)
$$

which proves the continuity of $\mu_{m}$

\subsection{The Min - Max principle}

In the sequel, we will study exclusively the eigenvalues of the operator $A(\omega)$ which do not belong to the essential spectrum $\sigma_{e s s}(\omega)$. The set of these eigenvalues will be denoted by $\sigma_{d}(\omega)$.

From (4.6) and theorem 4.4, we deduce

$$
\sigma_{d}(\omega) \subset\left[-\Lambda(\omega),+\infty[\cap]-\infty,-\beta_{\infty}^{2}(\omega)[\right.
$$

Moreover, by theorem $4.5, \sigma_{d}(\omega)$ is a finite set and each eigenvalue $\lambda$ of $\sigma_{d}(\omega)$ has finite multiplicity.

In the sequel, we denote by $N(\omega)$ the number of eigenvalues of $\sigma_{d}(\omega)$, each of them counted a number of times equal to its multiplicity. We will say that $N(\omega)$ is the "number of guided modes at the pulsation $\omega "$. 
The way to study the eigenvalues of $\sigma_{d}(\omega)$ is very classical and is based on the Min-Max principle (cf. [23]). Let us set :

$$
S_{1}(\omega)=\inf _{\varphi \in H^{1}(\Omega), \varphi \neq 0} \frac{a(\omega ; \varphi, \varphi)}{|\varphi|_{\Omega}^{2}}
$$

and for every integer $m>1$ :

$$
S_{m}(\omega)=\sup _{V \in \mathcal{V}_{m-1}(\Omega)} \inf _{\varphi \in \mathcal{V}^{\prime}, \varphi \neq 0} \frac{a(\omega ; \varphi, \varphi)}{|\varphi|_{\Omega}^{2}}
$$

where

- $a(\omega ; \varphi, \varphi)$ is defined by $(4.1)$

- $\mathcal{V}_{m}(\Omega)$ denotes the set of all m-dimensional subspaces of $H^{1}(\Omega)$

- For $V \subset H^{1}(\Omega): V^{\perp}=\left\{\psi \in H^{1}(\Omega) ; \int_{\Omega} \psi \xi d x d s=0, \forall \xi \in V\right\}$

One can use equivalently the following formula ( $\mathrm{cf}[4]$ )

$$
S_{m}(\omega)=\inf _{V \in \mathcal{V}_{m}(\Omega)} \sup _{\varphi \in V, \varphi \neq 0} \frac{a(\omega ; \varphi, \varphi)}{|\varphi|_{\Omega}^{2}}
$$

Then we have the

Proposition 4.1

$$
\begin{array}{lll}
\text { (i) } & -\Lambda(\omega) \leq S_{1}(\omega) \leq \ldots S_{m}(\omega) \leq-\beta_{\infty}^{2}(\omega), & \forall m . \\
\text { (ii) } \quad \sigma_{d}(\omega)=\left\{S_{m}(\omega) ; m \leq N(\omega)\right\} . & \\
\text { (iii) } & S_{m}(\omega)=-\beta_{\infty}^{2}(\omega), & \forall m>N(\omega) .
\end{array}
$$

In other words, we have

- If $S_{m}(\omega)<-\beta_{\infty}^{2}(\omega)$ then $N(\omega) \geq m$

- If $S_{m}(\omega)=-\beta_{\infty}^{2}(\omega)$ then $N(\omega)<m$

In the sequel, we will use extensively this characterization of $N(\omega)$. 


\section{Mathematical study of the dispersion curves}

\subsection{Preliminary results}

In this section, we study general properties of the dispersion curves and we present some techniques of comparison which will be used in the sequel.

\subsubsection{General properties of the functions $S_{m}(\omega)$}

The functions $S_{m}(\omega)$ are monotonic. Indeed, we have the

Lemma 5.1 The functions $\omega \longmapsto S_{m}(\omega), \omega \in \mathbb{R}^{+}$, are non-increasing.

Proof: This result is obvious since, for every $\varphi \in H^{1}(\Omega), \varphi \neq 0$, the function

$$
\omega \longmapsto \frac{a(\omega ; \varphi, \varphi)}{|\varphi|_{\Omega}^{2}}
$$

is non-increasing.

Moreover we have the following regularity result :

Lemma 5.2 The functions $\omega \longmapsto S_{m}(\omega)$ are continuous and almost everywhere differentiable.

Proof: Suppose for example $\omega_{1}<\omega_{2}$ and let $\varphi \in H^{1}(\Omega)$ such that $\int_{\Omega} \varphi^{2} d x d y=1$. Then :

$$
0 \leq a\left(\omega_{1} ; \varphi, \varphi\right)-a\left(\omega_{2} ; \varphi, \varphi\right)=\left(\omega_{1}^{2}-\omega_{2}^{2}\right) \int_{\Gamma_{F}} \varphi^{2} d x
$$

By lemma 4.1. and by the continuity of the trace operator from $H^{1}(\Omega)$ into $\Gamma_{F}$, there exist two constants $C_{1}$ and $C_{2}$ such that :

$$
\int_{\Gamma_{F}} \varphi^{2} d x \leq C_{1} a(\omega ; \varphi, \varphi)+C_{2} \quad \forall \omega \leq \omega_{2}
$$

Therefore, we have :

$$
a\left(\omega_{2} ; \varphi, \varphi\right) \leq a\left(\omega_{1} ; \varphi, \varphi\right) \leq\left(1+\left(\omega_{2}^{2}-\omega_{1}^{2}\right) C_{1}\right) a\left(\omega_{2} ; \varphi, \varphi\right)+\left(\omega_{2}^{2}-\omega_{1}^{2}\right) C_{2}
$$

and consequently :

$$
S_{m}\left(\omega_{2}\right) \leq S_{m}\left(\omega_{1}\right) \leq\left(1+\left(\omega_{2}^{2}-\omega_{1}^{2}\right) C_{1}\right) S_{m}\left(\omega_{2}\right)+\left(\omega_{2}^{2}-\omega_{1}^{2}\right) C_{2}
$$

This inequality proves that the function $S_{m}$ is continuous and locally Lipschitz. The lemma follows. 
Remark 5.1 In fact, we can prove that $A(\omega)$ is a selfadjoint holomorphic family of type $A$, in the sense of Kato (cf.[15]).

Consequently, the functions $\omega \longmapsto S_{m}(\omega)$ are in fact piecewise analytic. The singular points are, either points where the graphs of various eigenvalues intersect, or points where the mode is "cut-off", i.e where

$$
S_{n}(\omega)=-\beta_{\infty}^{2}(\omega)
$$

The singular points do not accumulate except may be at these cut-off values.

\subsubsection{Comparison principles}

Many comparison results can be directly deduced from the Min-Max principle and provide quantitative estimates for the functions $S_{m}(\omega)$. First we have the

Lemma 5.3 Let $h(x)$ and $\tilde{h}(x)$ be two positive functions satisfying (1.1) and such that:

$$
\tilde{h}(x) \leq h(x) \text { a.e. } \quad x \geq 0
$$

then (with obvious notations):

$$
\tilde{S}_{m}(\omega) \leq S_{m}(\omega) \quad \forall m \leq 1, \forall \omega \in \mathbb{R}
$$

Proof: Let $\Omega$ be the domain associated to the relief function $h$ :

$$
\Omega=\left\{(x, y) \in \mathbb{R}^{2} ; x>0,-h(x)<y<0\right\}
$$

and $\tilde{\Omega}$ the domain associated to $\tilde{h}$. Then, by (5.1), $\bar{\Omega} \subset \Omega$.

As we know that $S_{m}(\omega) \leq-\beta_{\infty}^{2}(\omega)<0$ we can restrict ourselves to work with functions $\varphi \in H^{1}(\Omega)$ satisfying :

$$
a(\omega ; \varphi, \varphi) \leq 0
$$

Let $\varphi$ be such a function and set $\bar{\varphi}=\left.\varphi\right|_{\tilde{\Omega}}$. Then $\bar{\varphi} \in H^{1}(\tilde{\Omega})$ and we remark that, with obvious notations :

$$
a(\omega ; \tilde{\varphi}, \bar{\varphi}) \leq \bar{a}(\omega ; \varphi, \varphi) \leq 0 \quad \text { and } \quad|\varphi|_{\Omega}^{2} \geq|\bar{\varphi}|_{\tilde{\Omega}}^{2}
$$

Consequently, if $\tilde{\varphi} \neq 0$, we deduce that :

$$
\frac{a(\omega ; \varphi, \varphi)}{|\varphi|_{\Omega}^{2}} \leq \frac{a(\omega ; \tilde{\varphi}, \tilde{\varphi})}{|\tilde{\varphi}|_{\tilde{\Omega}}^{2}}
$$

The lemma then follows by formula (4.21).

This lemma has the following immediate corollary : 
Corollary 5.1 Let $h(x)$ and $\tilde{h}(x)$ be two positine functions satisfying (1.1), (5.1) and:

$$
\tilde{h}_{\infty}=h_{\infty}
$$

then : $\bar{N}(\omega) \geq N(\omega), \quad \forall \omega \in \mathbb{R}$.

The two following comparison principles we will state are particular consequences of Jones' results (cf. [14]).

We consider again the decomposition $\bar{\Omega}=\bar{\Omega}_{e} \cup \bar{\Omega}_{i}$ of section 3.2. Notice that, for the results we will establish further, we can choose the abscissa $d$ of $\Sigma$ equal to $x_{0}$.

Consider the unbounded operator of $L^{2}\left(\Omega_{i}\right), A^{D}(\omega)$ with domain :

$$
\begin{array}{r}
D\left(A^{D}(\omega)\right)=\left\{\quad \varphi \in H^{1}\left(\Omega_{i}\right) ; \Delta \varphi \in L^{2}\left(\Omega_{i}\right), \frac{\partial \varphi}{\partial n}=0 \text { on } \Gamma_{B}^{i},\right. \\
\left.\frac{\partial \varphi}{\partial n}=\omega^{2} \varphi \text { on } \Gamma_{F}^{i}, \varphi=0 \text { on } \Sigma\right\}
\end{array}
$$

defined by :

$$
A^{D}(\omega) \varphi=-\triangle \varphi
$$

As in lemma 4.6 , we can prove that the spectrum of $A^{D}(\omega)$ consists in an increasing sequence of eigenvalues $\left(S_{m}^{D}(\omega)\right)$ tending to infinity, where $S_{m}^{D}(\omega)$ is defined by the following expression :

$$
S_{m}^{D}(\omega)=\inf _{V \in \mathcal{V}_{m}^{D}, p \in V, \varphi \neq 0} \sup _{\frac{a_{i}(\omega ; \varphi, \varphi)}{|\varphi|_{\Omega_{1}}^{2}}}
$$

where $\mathcal{V}_{m}^{D}$ denotes the set of all m-dimensional subspaces of the space $H_{D}^{1}\left(\Omega_{i}\right)$ :

$$
H_{D}^{1}\left(\Omega_{i}\right)=\left\{\varphi \in H^{1}\left(\Omega_{i}\right) ; \varphi \mid \Sigma=0\right\}
$$

and where :

$$
a_{i}(\omega ; \varphi, \varphi)=\int_{\Omega_{i}}|\nabla \varphi|^{2} d x d y-\omega^{2} \int_{\Gamma_{F}^{i}} \varphi^{2} d \sigma
$$

With these notations, we have the :

Lemma 5.4 $S_{m}(\omega) \leq S_{m}^{D}(\omega) \quad \forall m \geq 1, \forall \omega \in R$.

Proof: The space $H_{D}^{1}\left(\Omega_{i}\right)$ defined above can be considered as a subspace of $H^{1}(\Omega)$, since every function of $H_{D}^{1}\left(\Omega_{i}\right)$ can trivially be extended to a function of $H^{1}(\Omega)$.

Therefore, we have :

$$
\mathcal{V}_{m}^{D} \subset \mathcal{V}_{m}(\Omega), \forall m \geq 1
$$

and the lemma results from formula (4.21) and (5.3)

From this lemma and proposition 4.1 , we deduce the 
Corollary 5.2 If $S_{m}^{D}(\omega)<-\beta_{\infty}^{2}(\omega)$, then $N(\omega) \geq m$.

Lemma 5.4 gives a result of comparison between the eigenvalues of $A(\omega)$ and the eigenvalues of a "Dirichlet interior problem". Likewise, we can compare the eigenvalues of $A(\omega)$ to the one of a "Neumann interior problem".

Indeed, consider now the operator $A^{N}(\omega)$ with domain

$$
D\left(A^{N}(\omega)\right)=\left\{\varphi \in H^{1}\left(\Omega_{i}\right),\left.\left(\frac{\partial \varphi}{\partial y}-\omega^{2} \varphi\right)\right|_{\Gamma_{F}^{i}}=0,\left.\frac{\partial \varphi}{\partial n}\right|_{\Gamma_{B}^{i} \cup \Sigma}=0\right\}
$$

defined by :

$$
A^{N}(\omega) \varphi=-\triangle \varphi
$$

Like $A^{D}(\omega), A^{N}(\omega)$ has a purely discrete spectrum, consisting in an increasing sequence of eigenvalues $\left(S_{m}^{N}(\omega)\right)_{m \geq 1}$ tending to infinity. The expression of $S_{m}^{N}(\omega)$ we will use is the following :

$$
\left\{\begin{array}{l}
S_{1}^{N}(\omega)=\inf _{\varphi \in H^{1}\left(\Omega_{i}\right), \varphi \neq 0} \frac{a_{i}(\omega ; \varphi, \varphi)}{|\varphi|_{i}^{2}} \\
\text { and for } m>1 \\
S_{m}^{N}(\omega)=\sup _{V \in \mathcal{V}_{m-1}^{N}} \inf _{\varphi \in V^{\perp}, \varphi \neq 0} \frac{a_{i}(\omega ; \varphi, \varphi)}{|\varphi|_{\Omega_{i}}^{2}}
\end{array}\right.
$$

where $\mathcal{V}_{m}^{N}$ denotes the set of all m-dimensional subspaces of $H^{1}\left(\Omega_{i}\right)$ and where:

$$
V^{\perp}=\left\{\varphi \in V ; \int_{\Omega_{1}} \varphi \psi d x d y=0, \forall \psi \in H^{1}\left(\Omega_{i}\right)\right\}
$$

Then we have the

Lemma 5.5 $S_{m}(\omega) \geq \operatorname{Min}\left(S_{m}^{N}(\omega),-\beta_{\infty}^{2}(\omega)\right) \quad \forall \omega \in \mathbb{R}, \forall m \geq 1$

Proof

By formula (4.20), we have :

$$
S_{m}(\omega)=\sup _{V \in \mathcal{\nu}_{m-1}} \inf _{\varphi \in V^{2}, \varphi \neq 0} \frac{a_{i}(\omega ; \varphi, \varphi)+a_{e}(\omega ; \varphi, \varphi)}{|\varphi|_{\Omega_{i}}^{2}+|\varphi|_{\Omega_{e}}^{2}}
$$

where we have set :

$$
a_{e}(\omega ; \varphi, \varphi)=\int_{\Omega_{e}}|\nabla \varphi|^{2} d x d y-\omega^{2} \int_{\Gamma_{F}^{e}} \varphi^{2} d \sigma
$$

But a direct consequence of (3.7) is that, for every $\varphi$

$$
a_{e}(\omega ; \varphi, \varphi) \geq-\beta_{\infty}^{2}(\omega)|\varphi|_{\Omega_{e}}^{2}
$$


Therefore, if $\left.\varphi\right|_{\Omega_{i}} \neq 0$ :

$$
\frac{a_{i}(\omega ; \varphi, \varphi)+a_{e}(\omega ; \varphi, \varphi)}{|\varphi|_{\Omega_{i}}^{2}+|\varphi|_{\Omega_{e}}^{2}} \geq \operatorname{Min}\left(\frac{a_{i}(\omega ; \varphi, \varphi)}{|\varphi|_{\Omega_{i}}^{2}},-\beta_{\infty}^{2}(\omega)\right) .
$$

The lemma follows then from formulas (5.4)

As a consequence of the previous lemma and proposition 4.1, we have the :

Corollary 5.3 If $S_{m}^{N}(\omega) \geq-\beta_{\infty}^{2}(\omega)$, then $N(\omega)<m$. In particular, $N(\omega)$ is finite for every value of $\omega$.

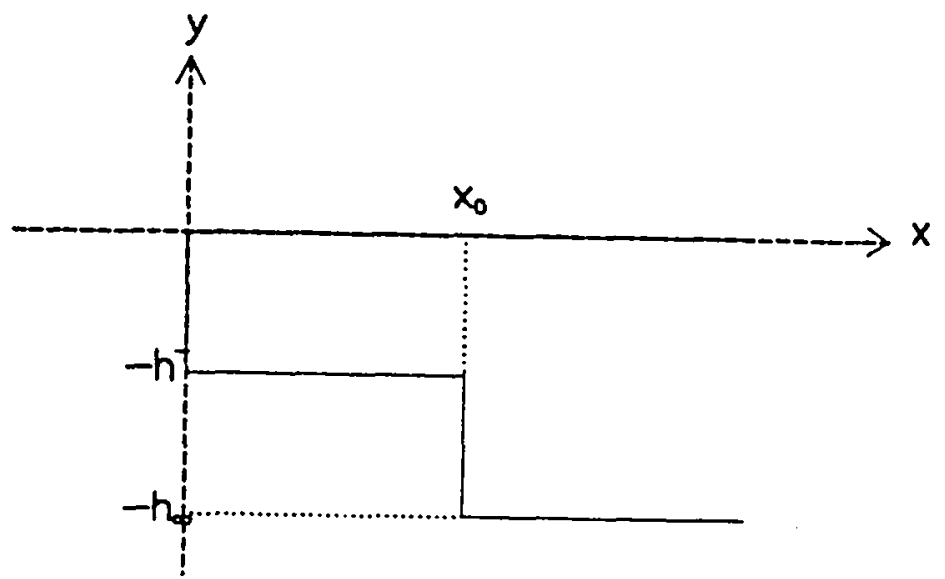

Figure 8: A shelf

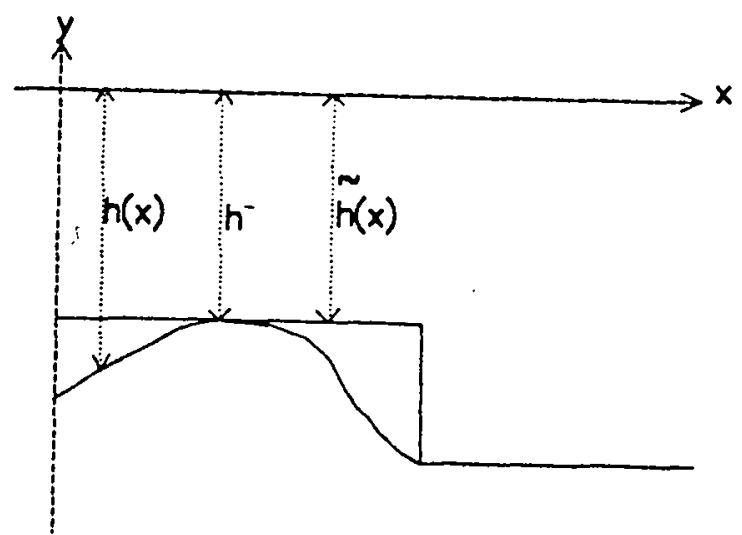

Figure 9: Comparison with a shelf

Remark 5.2 Corollaries 5.2 and 5.3 provide quantitative estimates for the case of a shelf (cf.[8]) (cf. figure 8). Indeed, if $d$ is chosen equal to the width of the shelf, $\Omega_{i}$ is a rectangle and the eigenvalues $S_{m}^{D}(\omega)$ and $S_{m}^{N}(\omega)$ can be calculated analytically.

Set :

$$
d(\omega)=\frac{x_{0}}{\pi}\left(\beta^{-}(\omega)^{2}-\beta_{\infty}(\omega)^{2}\right)^{1 / 2}
$$


wherr: $\mathrm{B}^{-}(\omega)$ is the solution of the equation:

$$
\beta \tanh \left(\beta h^{-}\right)=\omega^{2}
$$

and $h^{-}$is the depth above the shelf.

Then the results can be summarized as follows:

(i) If $m<d(\omega) \leq m+\frac{1}{2}$ then $m \leq N(\omega) \leq m+1$

(ii) If $m+\frac{1}{2}<d(\omega) \leq m+1$ then $N(\omega)=m+1$

Since $d(0)=0$ and $\lim _{\omega \rightarrow+\infty} d(\omega)=0$, it results form (i) that there is at most. one: gnided mode at low frequency $(\omega \rightarrow 0)$ and high frequency $(\omega \rightarrow+\infty)$.

By corollary 5.1, this remains true for every profile $h$ such that $h^{-}>0$ (cf. figure 9). Indecd, if $\bar{h}$ is defined by:

$$
\tilde{h}(x)=h^{-} \text {if } x<x_{0} \text { and } \tilde{h}(x)=h_{\infty} \text { if } x>x_{0},
$$

then $\tilde{h}(x) \leq h(x)$ p.p. $x>0$, and consequently:

$$
\bar{N}(\omega) \geq N(\omega) \text { for cvery } \omega
$$

Howewer, these results will be established by other methods in the following section.

\subsection{Results for abitrary shapes}

In this section, we will establish various results which do not depend on the behavior of the depth function $h$ at $x=0$.

\subsubsection{Existence results}

We begin by a non existence result. Suppose that (see figure 10):

$$
h(x) \geq h_{\infty}, \forall x>0
$$

then, by lemma (4.3):

$$
\sigma(\omega)=\sigma_{e s s}(\omega)=\left[-\beta_{\infty}^{2}(\omega),+\infty[,\right.
$$

and consequently, there are no eigenvalues below the essential spectrum. Notice that, by Fourier transform, it is easy to prove that there are no eigenvalues, even in the essential spectrum.

So we have proved the :

Lemma 5.6 If $h^{-}=h_{\infty}$ (where $h^{-}$and $h_{\infty}$ are defined by (2.1)), $N(\omega)=0$ for every $\omega$. 


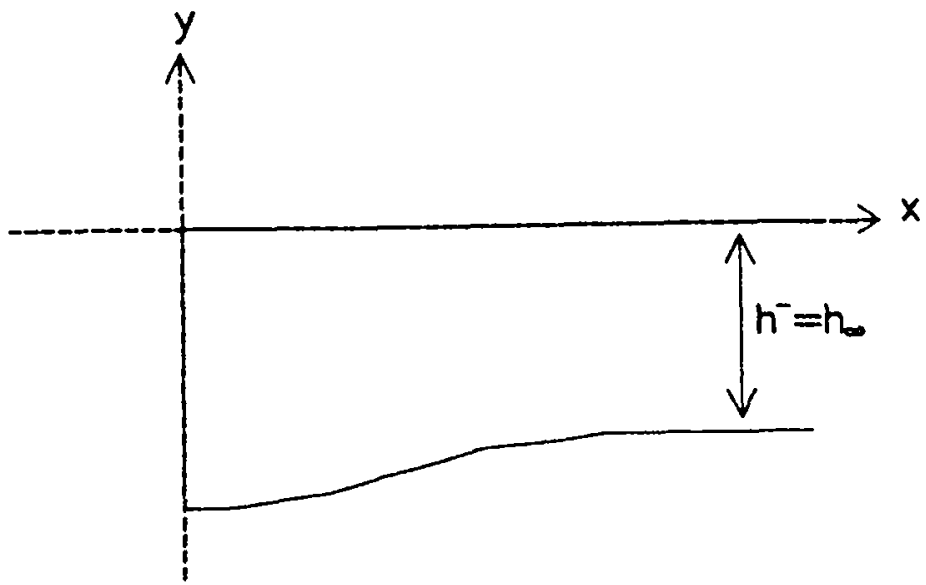

Figure 10: A case of non existence

Conversely, we have the :

Theorem 5.1 If $h^{-}<h_{\infty}$ (cf figure 11), then there exists a constant $\omega^{*} \geq 0$ such that:

$$
S_{1}(\omega)<-\beta_{\infty}^{2}(\omega) \text { for } \omega>\omega^{*}
$$

and consequently

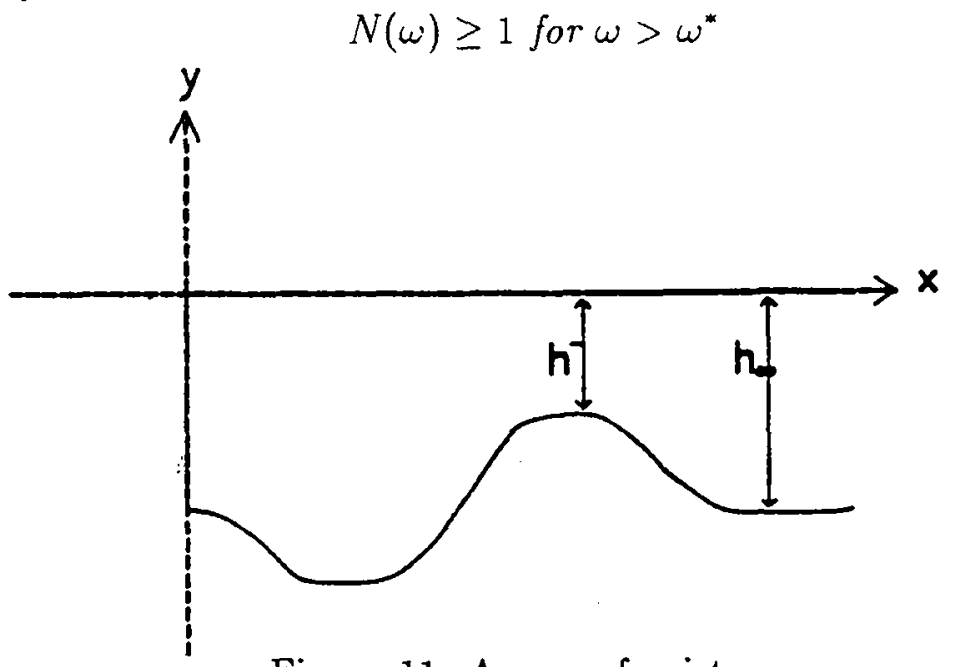

Figure 11: A case of existence

Proof: The idea of the proof is due to Garipov (cf [16]). We consider the test function $\varphi_{\alpha}$ defined by :

$$
\varphi_{\alpha}(x, y)=w(y) e^{-\alpha x}
$$

where $\alpha$ is a strictly positive constant and where :

$$
w(y)= \begin{cases}\cosh \left(\beta_{\infty}(\omega)\left(y+h_{\infty}\right)\right) & \text { if } y \geq-h_{\infty} \\ 1 & \text { if } y \leq-h_{\infty}\end{cases}
$$


Then we have :

$$
\begin{aligned}
& "(\omega ; \varphi, \varphi)+\beta_{\infty}^{2}(\omega)|\varphi|_{\Omega}^{2} \\
& =\int_{0}^{+\infty}\left\{\int_{-h(x)}^{0}\left(\left.1 \frac{d w}{d y}\right|^{2}+\alpha^{2} w^{2}+\beta_{\infty}^{2}(\omega) w^{2}\right) d y-\omega^{2} w^{2}(0)\right\} e^{-2 \alpha x} d x
\end{aligned}
$$

Moreover, by definition of $w$ :

$$
\begin{aligned}
\left(\frac{d w}{d y}\right)^{2}+\beta_{\infty}^{2}(\omega) w^{2} & =\beta_{\infty}^{2}(\omega) \cosh \left(2 \beta_{\infty}(\omega)\left(y+h_{\infty}\right)\right) & & \text { if } y \geq-h_{\infty} \\
& =\beta_{\infty}^{2}(\omega) & & \text { if } y \leq-h_{\infty}
\end{aligned}
$$

and consequently :

$$
\begin{array}{ll}
\int_{-h(x)}^{0}\left\{\left|\frac{d w}{d y}\right|^{2}+\beta_{\infty}^{2}(\omega) w^{2}\right\} d y= \\
= \begin{cases}\frac{1}{2} \beta_{\infty}(\omega)\left(\sinh \left(2 \beta_{\infty}(\omega) h_{\infty}\right)-\sinh \left(2 \beta_{\infty}(\omega)\left(h_{\infty}-h(x)\right)\right)\right) & \text { if } h(x) \leq h_{\infty} \\
\frac{1}{2} \beta_{\infty}(\omega)\left(\sinh \left(2 \beta_{\infty}(\omega) h_{\infty}\right)+\beta_{\infty}^{2}(\omega)\left(h(x)-h_{\infty}\right)\right) & \text { if } h(x) \geq h_{\infty}\end{cases}
\end{array}
$$

Using the identity :

$$
\frac{\beta_{\infty}(\omega)}{2} \sinh \left(2 \beta_{\infty}(\omega) h_{\infty}\right)-\omega^{2} \cosh ^{2}\left(\beta_{\infty}(\omega) h_{\infty}\right)=0
$$

which is derived from (2.2), and the inequality $w(y) \leq \cosh \left(\beta_{\infty}(\omega) h_{\infty}\right)$ we get :

$$
\left\{\begin{array}{c}
a(\omega ; \varphi, \varphi)+\beta_{\infty}^{2}(\omega)|\varphi|_{\Omega}^{2} \leq \frac{\alpha}{2} \cosh ^{2}\left(\beta_{\infty}(\omega) h_{\infty}\right) \\
-\frac{\beta_{\infty}(\omega)}{2} \int_{\gamma^{-}} \sinh \left(2 \beta_{\infty}(\omega)\left(h_{\infty}-h(x)\right)\right) e^{-2 \alpha x} d x \\
+\beta_{\infty}^{2}(\omega) \int_{\gamma^{+}}\left(h(x)-h_{\infty}\right) e^{-2 \alpha x} d x
\end{array}\right.
$$

where

$$
\begin{aligned}
& \gamma^{-}=\left\{x>0 ; h(x)<h_{\infty}\right\} \\
& \gamma^{+}=\left\{x>0 ; h(x)>h_{\infty}\right\}
\end{aligned}
$$

To prove (5.5), it suffices to show that the right hand side of (5.6) is strictly negative for $\omega$ large enough. By hypothesis, $h^{-}<h_{\infty}$. Therefore, the set

$$
\left\{x>0 ; h_{\infty}-h(x)>\frac{1}{2}\left(h_{\infty}-h^{-}\right)\right\}
$$

has a positive measure which we will denote by $\mu$. Hence as $\gamma^{-} \subset\left[0, x_{0}\right]$ :

$$
\forall \alpha \leq 1 \quad \int_{\gamma^{-}} \sinh \left(2 \beta_{\infty}(\omega)\left(h_{\infty}-h(x)\right)\right) d x \geq \mu e^{-x_{0}} \sinh \left(\beta_{\infty}(\omega)\left(h_{\infty}-h^{-}\right)\right)
$$


Since $\beta_{\infty}(\omega)$ tends to infinity like $\omega^{2}$ as $\omega$ tends to infinity, this integral increases exponentially with $\omega$. Therefore as soon as $\alpha \leq 1$, for $\omega$ great enough :

$$
\frac{1}{2} \int_{\gamma^{-}} \sinh \left(2 \beta_{\infty}(\omega)\left(h_{\infty^{-}}-h(x)\right)\right) e^{-2 \alpha x} d x-\beta_{\infty}(\omega) \int_{\gamma^{+}}\left(h(x)-h_{\infty}\right) e^{-2 \alpha x} d x>0
$$

To conclude, notice that for each fixed $\omega$ such that (5.7) holds, $\alpha$ can be choosen small enough for the right hand side of (5.6) to be strictly negative.

By a slight modification of the previous proof, we will show that, under an integral condition on $h$ which is explicited below, the fundamental mode exists at every positive frequency:

Theorem 5.2 If $h^{-}<h_{\infty}$ and if :

$$
\int_{0}^{+\infty}\left(h(x)-h_{\infty}\right) d x \leq 0
$$

then.

$$
S_{1}(\omega)<-\beta_{\infty}^{2}(\omega), \text { for every } \omega>0 .
$$

Proof : From formula (5.6), we deduce the following inequality :

$$
a(\omega ; \varphi, \varphi)+\beta_{\infty}^{2}(\omega)|\varphi|_{\Omega}^{2} \leq \frac{\alpha}{2} \cosh ^{2}\left(\beta_{\infty}(\omega) h_{\infty}\right)+\beta_{\infty}^{2}(\omega) \int_{0}^{+\infty}\left(h(x)-h_{\infty}\right) e^{-2 \alpha x} d x
$$

Then, by (5.8), for every non-zero value of $\omega$, we can choose the value of $\alpha$ small enough to make the term at the right of this inequality strictly negative.

We will prove now that condition (5.8) is in fact necessary for the fundamental mode to exist at arbitrarily small frequencies :

Theorem 5.3 Suppose that there is a sequence $\left(\omega_{n}\right)_{n \in \mathbb{N}}$ of $\mathrm{R}^{+}$such that

$$
\left\{\begin{array}{l}
\omega_{n} \longrightarrow 0, \\
S_{1}\left(\omega_{n}\right)<-\beta_{\infty}^{2}\left(\omega_{n}\right), \forall n \in N,
\end{array}\right.
$$

then the depth profile $h$ necessarily satisifies condition (5.8). Consequently, (5.9) holds.

Proof: By hypothesis and by lemma (5.6), we have :

$$
S_{1}^{N}\left(\omega_{n}\right)<-\beta_{\infty}^{2}\left(\omega_{n}\right), \forall n \in N
$$

where $S_{1}^{N}(\omega)$ is defined by (5.4). Consequently, there exists a sequence of functions of $H^{1}\left(\Omega_{i}\right),\left(\varphi_{n}\right)_{n \in N}$, such that :

$$
a_{i}\left(\omega_{n} ; \varphi_{n}, \varphi_{n}\right)+\beta_{\infty}^{2}\left(\omega_{n}\right)\left|\varphi_{n}\right|_{\Omega_{i}}^{2}<0
$$


We can choose the functions $\varphi_{n}$ normalized as follows :

$$
\int_{\Gamma_{F}^{2}} \varphi_{n}^{2} d \sigma=1
$$

From (5.10) and (5.11), we deduce :

$$
\int_{\Omega_{1}}\left\{\left|\nabla \varphi_{n}\right|^{2}+\beta_{\infty}\left(\omega_{n}\right)^{2} \varphi_{n}^{2}\right\} d x d y<\omega_{n}^{2}
$$

From this estimate, it results that the sequence $\left(\nabla \varphi_{n}\right)_{n \in \mathbb{N}}$ converges strongly to 0 in $\left(L^{2}\left(\Omega_{i}\right)\right)^{2}$ and that :

$$
\int_{\Omega_{i}} \varphi_{n}^{2} d x d y<\frac{\omega_{n}^{2}}{\beta_{\infty}\left(\omega_{n}\right)^{2}}
$$

Since $\beta_{\infty}(\Omega)$ is equivalent to $\frac{\omega}{\sqrt{h_{\infty}}}$ as $\omega$ tends to $0,(5.12)$ proves that the sequence $\left(\varphi_{n}\right)_{n \in \mathbb{N}}$ is bounded in $L^{2}\left(\Omega_{i}\right)$, and therefore in $H^{1}\left(\Omega_{i}\right)$.

By classical compactness results, there exists a subsequence still denoted by $\left(\varphi_{n}\right)$, which converges strongly in $H^{1}\left(\Omega_{i}\right)$ to a constant function $\varphi$. By $(5.11), \varphi=\frac{1}{\sqrt{\alpha}}$ and by (5.12).

$$
\frac{1}{d} \int_{0}^{d} h(x) d x \leq h_{\infty}
$$

which is equivalent to $(5.8)$.

Finally, we will prove that only the fundamental mode can exist at low frequency :

Theorem 5.4 There exists a constant $\omega_{*}>0$ such that :

$$
S_{2}(\omega)=-\beta_{\infty}^{2}(\omega) \text { for } \omega \leq \omega_{*}
$$

and therefore:

$$
N(\omega) \leq 1 \text { for } \omega \leq \omega_{*}
$$

Proof: By lemma 5.5, it is sufficient to prove that:

$$
S_{m}^{N}(\omega)=-\beta_{\infty}^{2}(\omega) \text { for } \omega \leq \omega_{*} .
$$

Suppose that is false. Then there exists a sequence $\omega_{p}, \omega_{p} \longrightarrow 0$ and a sequence $\left(\varphi_{p}\right), \varphi_{p} \in$ $H^{1}\left(\Omega_{i}\right)$, such that :

$$
\left\{\begin{array}{l}
\int_{\Gamma_{F}} \varphi_{p}(x, 0) d x=0 \\
\int_{\Gamma_{F}} \varphi_{p}^{2}(x, 0) d x=1 \\
a_{i}\left(\omega_{p} ; \varphi_{p}, \varphi_{p}\right)+\beta_{\infty}\left(\omega_{p}\right)^{2}\left|\varphi_{p}\right|_{\Omega_{i}}^{2}<0
\end{array}\right.
$$


From the last inequality, its results that $\nabla \varphi_{p} \rightarrow 0$ strongly in $\left(L^{2}\left(\Omega_{i}\right)\right)^{2}$ and that $\varphi_{p}$ is bounded in $I_{\nu}^{2}\left(\Omega_{i}\right)$ (since $\beta_{\infty_{0}}\left(\omega_{p}\right) \sim \frac{\omega_{p}}{\sqrt{h_{\infty}}}$ when $p \rightarrow+\infty$ ). Consequently, $\varphi_{p}$ converges to $\varphi$, weakly in $H^{1}\left(\Omega_{i}\right)$ and strongly in $L^{2}\left(\Omega_{i}\right)$ and $\varphi$ must satisfy :

$$
\nabla \varphi=0, \int_{\Gamma_{F}^{i}} \varphi^{2}(x, 0) d x=1 \text { and } \int_{\Gamma_{F}^{i}} \varphi(x, 0) d x=0
$$

which is impossible

\subsubsection{Properties of the fundamental mode}

We can now establish a Krein-Rutman type result :

Proposition 5.1 If $S_{1}(\omega)<-\beta_{\infty}^{2}(\omega)$, then :

(i) $S_{1}(\omega)$ is a simple eigenvalue of $A(\omega)$.

(ii) The associated eigenfunction $\varphi$ can be taken such that $\varphi(x) \geq 0$ a.e. in $\Omega$.

Proof We proceed as Manes and Micheletti ([18]). Let $\varphi$ be an eigenfunction associated to $S_{1}(\omega)$ and set $\varphi^{+}=\max (\varphi, 0)$ and $\varphi^{-}=\min (\varphi, 0)$. Then, by [25], $\varphi^{+}$and $\varphi^{-}$belong to $H^{1}(\Omega)$ and we have :

$$
S_{1}(\omega)=\frac{a(\omega ; \varphi, \varphi)}{|\varphi|_{\Omega}^{2}}=\frac{a\left(\omega ; \varphi^{+}, \varphi^{+}\right)+a\left(\omega ; \varphi^{-}, \varphi^{-}\right)}{\left|\varphi^{+}\right|_{\Omega}^{2}+\left|\varphi^{-}\right|_{\Omega}^{2}}
$$

Suppose neither $\varphi^{+}$nor $\varphi^{-}$are identically equal to zero, and suppose, for example :

$$
\frac{a\left(\omega ; \varphi^{+}, \varphi^{+}\right)}{\left|\varphi^{+}\right|_{\Omega}^{2}} \geq \frac{a\left(\omega ; \varphi^{-}, \varphi^{-}\right)}{\left|\varphi^{-}\right|_{\Omega}^{2}}
$$

Then, by (5.13) and (4.19) :

$$
S_{1}(\omega)=\frac{a(\omega ; \varphi, \varphi)}{|\varphi|_{\Omega}^{2}}=\frac{a\left(\omega ; \varphi^{+}, \varphi^{+}\right)}{|\varphi|_{\Omega}^{2}}=\frac{a\left(\omega ; \varphi^{-}, \varphi^{-}\right)}{|\varphi|_{\Omega}^{2}}
$$

and consequently, $\varphi^{+}$and $\varphi^{-}$are eigenfunctions associated to $S_{1}(\omega)$. Therefore, $\varphi^{+}$and $\varphi^{-}$are analytical in $\Omega$.

But since $\varphi^{+} \varphi^{-}$is identically 0 in $\Omega$, one of the two functions $\varphi^{+}$or $\varphi^{-}$must vanish identically, which is in contradiction with the hypothesis.

So we have proved that every eigenfunction $\varphi$ associated to $S_{1}(\omega)$ can be taken every. where non-negative.

Now suppose $\varphi$ and $\psi$ are two eigenfunctions associated to $S_{1}(\omega)$ and set :

$$
\begin{aligned}
& A^{+}=\{\alpha \in \mathbb{R} ; \alpha \varphi+\psi \geq 0 \text { a.e. in } \Omega\} \\
& A^{-}=\{\alpha \in \mathbb{R} ; \alpha \varphi+\psi \leq 0 \text { a.e. in } \Omega\}
\end{aligned}
$$


Then $A^{+}$and $A^{-}$are closed subsets of $\mathrm{R}$ and by the first part of the proof : $A^{+} \cup A^{-}=\mathbb{R}$. Consequently, $A^{+} \cap A^{-} \neq \emptyset$ and $\varphi$ and $\psi$ are linearly dependent. This proves (i).

From theorem 5.4, we deduce (cf. [15]) the

Corollary 5.4 The function $\omega \longmapsto S_{1}(\omega)$ is holomorphic in cvery interval $I \subset \mathbf{R}$ such that :

$$
S_{1}(\omega)<-\beta_{\infty}^{2}(\omega), \forall \omega \in I
$$

\subsection{The case of the cliff}

We will establish in this section some high frequency results concerning the case of a cliff, i.e.

$$
h^{-}>0
$$

or, more generally :

$$
h(x) \geq H x^{\gamma} \text { for small } x, \text { with } 0<\gamma<1 \text { and } H>0
$$

Under assumption (5.14), we have already proved (see the end of section 5.1) by comparison results that $N\left(\omega^{\prime}\right) \leq 1$ for large $\omega$.

We will prove this result again, by a technique which can be generalized to the case of a profile satisfying (5.15).

We establish first a preliminary lemma :

Lemma 5.7 Suppose there exists a sequence $\omega_{p}, \omega_{p} \rightarrow+\infty$, such that :

$$
S_{2}\left(\omega_{p}\right)<-\beta_{\infty}^{2}\left(\omega_{p}\right), \quad \forall p \in \mathbb{N},
$$

then, there exists a sequence $\left(\varphi_{p}\right), \varphi_{p} \in H^{1}\left(\Omega_{i}\right)$, such that:

$$
\begin{aligned}
& \text { (i) } \int_{0}^{d} \varphi_{p}(x, 0) d x=0 \\
& \text { (ii) } \int_{0}^{d} \varphi_{p}^{2}(x, 0) d x=1 \\
& \text { (iii) } \int_{\Omega_{i}}\left\{\left|\frac{\partial \varphi_{p}}{\partial y}\right|^{2}+\varphi_{p}^{2}\right\} d x d y \leq 1 \\
& \text { (iv) } \int_{\Omega_{1}}\left|\frac{\partial \varphi_{p}}{\partial x}\right|^{2} d x d y \leq \beta_{p} \int_{0}^{d}\left(\tanh \left(\beta_{p} h_{\infty}\right)-\tanh \left(\beta_{p} h(x)\right)\right) \varphi_{p}^{2}(x, 0) d x
\end{aligned}
$$

where $\beta_{p}=\beta_{\infty}\left(\omega_{p}\right)$. 
Proof : As in the proof of theorem 5.4, we can consider $S_{2}^{N}\left(\omega_{p}\right)$ instead of $S_{2}\left(\omega_{p}\right)$ and we deduce the existence of a sequence $\left(\varphi_{p}\right), \varphi_{p} \in H^{1}\left(\Omega_{i}\right)$, satisfying (i), (ii) and:

$$
\int_{\Omega_{1}}\left\{\left|\nabla \varphi_{p}\right|^{2}+\beta_{p}^{2} \varphi_{p}^{2}\right\} d x d y<\omega_{p}^{2}
$$

Moreover, by (3.7), we have:

$$
\int_{\Omega_{1}}\left\{\left.1 \frac{\partial \varphi_{p}}{\partial y}\right|^{2}+\beta_{p}^{2} \varphi_{p}^{2}\right\} d x d y \geq \beta_{p} \int_{0}^{d} \tanh \left(\beta_{p} h(x)\right) \varphi_{p}^{2}(x, 0) d x
$$

From (5.16) and (5.17), we deduce (iv).

The result is finally obtained by a scaling. Indeed, if we set :

$$
\tilde{\varphi}_{p}(x, y)=\varphi_{p}\left(x, \frac{y}{\beta_{p}}\right)
$$

one can easily check that $\left(\tilde{\varphi}_{p}\right)$ satifies all required properties.

We are now ready to prove the

Theorem 5.5 If the depth function $h$ satisfies (5.14) or (5.15), then there exists a value $\omega_{c}$ such that :

$$
S_{2}(\omega)=-\beta_{\infty}^{2}(\omega) \text { for } \omega \geq \omega_{c}
$$

Proof: By lemma 5.10, there exists a sequence $\varphi_{p}$ satisfying (i), (ii), (iii) and (iv).

1. Suppose first that $h$ satisfies (5.14). Then, we deduce from (iv) the following inequality :

$$
\begin{aligned}
\int_{\Omega_{i}}\left|\frac{\partial \varphi_{p}}{\partial x}\right|^{2} d x d y & \leq \beta_{p}\left(\tanh \left(\beta_{p} h_{\infty}\right)-\tanh \left(\beta_{p} h^{-}\right)\right) \\
& \leq 2 \beta_{p} e^{-2 \beta_{p} h}
\end{aligned}
$$

Joined with (iii), this proves that $\left(\varphi_{p}\right)$ is bounded in $H^{1}\left(\Omega_{i}\right)$. Consequently, there exists a subsequence still denoted by $\left(\varphi_{p}\right)$ which converges to some $\varphi \in H^{1}\left(\Omega_{i}\right)$ weakly in $H^{1}\left(\Omega_{i}\right)$. By compacity, $\varphi$ satifies :

$$
\int_{0}^{d} \varphi(x, 0) d x=0 \text { and } \int_{0}^{d} \varphi^{2}(x, 0) d x=1
$$

But this is in contradiction with (5.18), which implies that $\frac{\partial \varphi}{\partial x}$ is identically zero.

2. Suppose now that $h$ satisfies (5.15) for $x<\alpha$ where $0<\alpha<1$ and set :

$$
h_{*}=\inf _{x>\alpha} h(x)
$$


Then, we deduce from (iv) :

$$
\int_{\Omega_{i}}\left|\frac{\partial \varphi_{p}}{\partial x}\right|^{2} d x d y \leq 2 \beta_{p} \int_{u}^{1} e^{-2 \beta_{p} H x^{\prime \gamma}} \varphi_{p}^{2}(x, 0) d x+2 \beta_{p} e^{-2 \beta_{p} h *}
$$

Consequently, by the Holder's inequality, we obtain for $q>2$ :

$$
\int_{\Omega_{i}}\left|\frac{\partial \varphi_{p}}{\partial x}\right|^{2} d x d y \leq\left\|g_{p}\right\|_{L^{r}(0,1)}^{2}\left\|\varphi_{p}\right\|_{L^{\eta}\left(\Gamma_{F}^{\prime}\right)}^{2}+2 \beta_{p} e^{-2 \beta_{p} h_{*}}
$$

where :

$$
\begin{aligned}
& g_{p}(x)=2 \beta_{p} e^{-2 \beta_{p} H x^{\gamma}}, \\
& r=\frac{q}{q-2} .
\end{aligned}
$$

Moreover, setting $y=2 r \beta_{p} H x^{\gamma}$, we obtain :

$$
\left\|g_{p}\right\|_{L^{r}(0,1)} \leq 2 \beta_{p}\left(2 \beta_{p} H r\right)^{-\frac{1}{\gamma r}}\left[\frac{1}{\gamma} \int_{0}^{+\infty} e^{-y} y^{\frac{1-\gamma}{\gamma}} d y\right]^{1 / r}
$$

and consequently, if $1-\frac{1}{\gamma r}<0$ (i.e. if $q>\frac{2}{1-\gamma}$ ),

$$
\left\|g_{p}\right\|_{L^{r}(0,1)} \longrightarrow_{p \rightarrow+\infty} 0
$$

Then we notice that, by the continuity of the trace application from $H^{1}\left(\Omega_{i}\right)$ into $H^{1 / 2}\left(\Gamma_{F}^{i}\right)$ and of the injection $H^{1 / 2}\left(\Gamma_{F}^{i}\right) \hookrightarrow L^{q}\left(\Gamma_{F}^{i}\right)$, there exists a constant $K$ such that :

$$
\left\|\varphi_{p}\right\|_{L^{q}\left(\Gamma_{F}^{i}\right)}^{2} \leq K\left\|\varphi_{p}\right\|_{H^{1}\left(\Omega_{i}\right)}^{2}
$$

Finally, by (5.18) and (iii) we have :

$$
\left|\frac{\partial \varphi_{p}}{\partial x}\right|_{\Omega_{1}}^{2} \leq K\left\|g_{p}\right\|_{L^{r}(0,1)}\left(1+\left|\frac{\partial \varphi_{p}}{\partial x}\right|_{\Omega_{1}}^{2}\right)+2 \beta_{p} e^{-2 \beta_{p} h}
$$

which proves that $\left|\frac{\partial \dot{\varphi}_{p}}{\partial x}\right|_{\Omega_{i}}$ tends to zero as $p \rightarrow+\infty$. We conclude then as in the first part of the proof.

By theorems 5.1 and 5.5, if $h^{-}<h_{\infty}$, only the fundamental mode exists at high frequency. We will now study the asymptotic behavior of the fundamental dispersion curve $\omega \longmapsto$ $S_{1}(\omega)$. As a direct consequence of lemma 4.3 , we have the

Lemma 5.8 If the depth function $h$ satifies (5.14), then

$$
0 \leq-\left(S_{1}(\omega)+\beta_{\infty}(\omega)^{2}\right) \leq 4 \omega^{4} e^{-4 \omega^{2} h^{-}}\left(1-4 e^{-2 \omega^{2} h^{-}}\right)^{-1}
$$

for large $\omega$ 
Proof : Indeed, we have :

$$
-\beta^{-}(\omega)^{2} \leq S_{1}(\omega) \leq-\beta_{\infty}(\omega)^{2}
$$

where

$$
\beta^{-}(\omega) \tanh \left(\beta^{-}(\omega) h^{-}\right)=\beta_{\infty}(\omega) \tanh \left(\beta_{\infty}(\omega) h_{\infty}\right)=\omega^{2}
$$

and :

$$
\omega^{2}<\beta_{\infty}(\omega)<\beta^{-}(\omega)<\omega^{2}\left(1-2 e^{-2 \omega^{2} h^{-}}\right)^{-1}
$$

Remark 5.3 Let $\varphi(\omega)$ be an eigenfunction associated to $S_{1}(\omega)$ (for $\omega$ large enough). By lemma 3.2, we know that $\varphi(\omega)$ decreases exponentially as $x \longrightarrow+\infty$ as $e^{-\alpha_{0}(\omega) x}$ where : $\alpha_{0}(\omega)=\left(-\beta_{\infty}^{2}(\omega)-S_{1}(\omega)\right)^{1 / 2}$.

By the previous lemma, we see that the rate of decay $\alpha_{0}(\omega)$ tends to zero as $\omega$ tends to infinity. This means that the energy becames less and less confined as the frequency increases.

In the general case, we have the

Lemma 5.9 If the depth function h satisfies (5.15) then :

$$
\frac{\left|S_{1}(\omega)+\beta_{\infty}^{2}(\omega)\right|}{\omega^{q}} \longrightarrow 0 \quad \text { as } \omega \rightarrow+\infty
$$

for :

$$
q>\frac{8 \gamma-4}{\gamma}
$$

Proof : To prove this result, we compare the profile $h$ with profiles $h_{\theta}$ such that :

$$
h_{\theta}(x)=\left\{\begin{array}{l}
x \tan \theta \text { if } x \leq x_{\theta} \\
h_{\infty, \theta}=x_{\theta} \tan \theta \text { if } x \geq x_{\theta}
\end{array}\right.
$$

Indeed, if $\theta$ is near enough from $\frac{\pi}{2}$, we have :

$$
h(x) \geq h_{\theta}(x) \text { a.e }
$$

provided that : $x_{\theta} \tan \theta=H x_{\theta}^{\gamma}$. (see figure 12)

So, $x_{\theta}$ and $h_{\infty, \theta}$ are defined by :

$$
\left\{\begin{array}{l}
x_{\theta}=(H \tan \theta)^{\frac{-1}{1-\gamma}} \\
h_{\infty, \theta}=H^{\frac{-1}{1-\gamma}} \tan \theta^{\frac{-\gamma}{1-\gamma}}
\end{array}\right.
$$




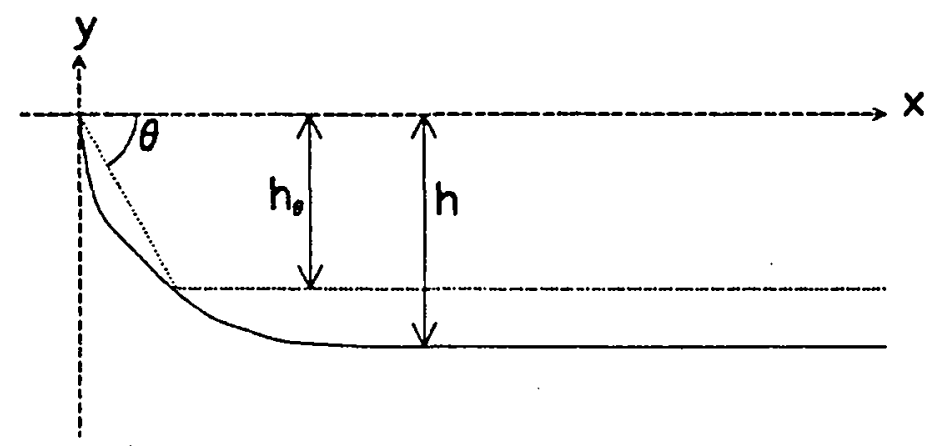

Figure 12: The relief $h_{\theta}$

From (5.20), we deduce by lemma 4.3 :

$$
-\beta_{\theta}^{2}(\omega) \leq S_{1}(\omega) \leq-\beta_{\infty}^{2}(\omega), \forall \omega \in \mathbb{R}^{+},
$$

where $\beta_{\theta}(\omega)$ is the positive solution of :

$$
\beta_{\theta} \tanh \left(\beta_{\theta} h_{\infty, \theta}\right)=\frac{\omega^{2}}{\sin ^{2} \theta} .
$$

As in the proof of lemma 5.11, a simple calculation provides then the following estimate

$$
\frac{\left|S_{1}(\omega)+\beta_{\infty}^{2}(\omega)\right|}{\omega^{q}} \leq \omega^{4-q} \frac{1}{1-\varepsilon}\left(\frac{1-\sin ^{4} \theta}{\sin ^{4} \theta}+\varepsilon\right)
$$

with $\varepsilon=4 e^{-2 \omega^{2} h_{\infty}, \theta}$

That inequality holds for every angle $\theta$ near enough from $\frac{\pi}{2}$. In particular, we can choose, for large $\omega:$

$$
\theta=\frac{\pi}{2}-\omega^{-p}
$$

for some real $p>0$. For that choice, we get by (5.21):

$$
h_{\infty, \theta} \sim H^{\frac{-1}{1-\gamma} \omega^{\frac{-p \gamma}{1-\gamma}}} \text { as } \omega \rightarrow+\infty
$$

Consequently, if

$$
2-\frac{p \gamma}{1-\gamma}>0
$$

then $\epsilon$ tends exponentially to zero as $\omega$ tends to infinity.

Moreover

$$
\frac{1-\sin ^{4} \theta}{\sin ^{4} \theta} \sim 2 \omega^{-2 p} \text { as } \omega \rightarrow+\infty
$$


Finally, by $(5.22), \frac{\left|S_{1}(\omega)+\beta_{x}^{2}(\omega)\right|}{\omega^{q}}$ tends to zero as $\omega \rightarrow+\infty$ if

$$
4-q-2 p<0
$$

To find an integer $p$ satisfying (5.23) and (5.25), we must have :

$$
\frac{4-q}{2}<\frac{2(1-\gamma)}{\gamma}
$$

Remark 5.4 (i) For $\gamma<\frac{1}{2}$, by the previous lemma:

$$
\left|S_{1}(\omega)+\beta_{\infty}^{2}(\omega)\right| \longrightarrow 0 \quad \text { as } \omega \rightarrow+\infty
$$

It means again that the energy becomes less and less confined when the frequency increases.

(ii) Notice that for every $\gamma<1$

$$
\frac{\left|S_{1}(\omega)+\beta_{\infty}^{2}(\omega)\right|}{\omega^{4}} \longrightarrow 0 \quad \text { as } \omega \rightarrow+\infty
$$

\subsection{The case of the sloping beach}

We suppose now that the profile $h$ is such that

$$
\lim _{x \rightarrow 0} \frac{h(x)}{x}=\tan \alpha
$$

with

$$
0<\alpha<\frac{\pi}{2}
$$

Firstly, we will prove that there are in that case at least $M(\alpha)$ guided modes at high frequency, where $M(\alpha)$ is the number of guided modes for the infinite sloping beach studied by Ursell ([30]).

Conversely, our conjecture is that the number $N(\omega)$ of guided modes at high frequency remains bounded as the frequency increases, but we succeed to prove it only if

$$
\liminf _{x \rightarrow 0} \frac{h(x)}{x} \geq \tan \frac{\pi}{4}
$$

Let us first briefly establish various results concerning the case of an infinite sloping beach with angle $\alpha$.(cf. figure 2) We denote by $h_{\alpha}$ the corresponding profile

$$
h_{\alpha}(x)=x \tan \alpha, \forall x>0,
$$


and by $\Omega_{\alpha}$ the associated domain

$$
\Omega_{\alpha}=\left\{(x, y) \in \mathbb{R}^{2} ; x>0 \text { and }-h_{\alpha}(x)<y<0\right\}
$$

Notice that $h_{\alpha}$ does not satisfy hypothesis $(1.1,($ ii $))$.

However we can define an operator $A_{\alpha}(\omega)$ as we defined $A(\omega)$ (cf. section 3.1), by replacing in the definition $\Omega$ by $\Omega_{\alpha}$. Then we have the

Lemma 5.10 The operator $A_{\alpha}(\omega)$ is self-adjoint and

$$
\left(A_{\alpha}(\omega) \varphi, \varphi\right) \geq\left(\frac{\omega^{2}}{\sin \alpha}\right)^{2}|\varphi|_{\Omega_{\alpha}}^{2} \quad, \forall \varphi \in D\left(A_{\alpha x}(\omega)\right) .
$$

Proof : Using the same change of coordinates as in the proof of lemma 4.3, we obtain likewike

$$
\left(A_{\alpha}(\omega) \varphi, \varphi\right) \geq \sin ^{2} \alpha \int_{0}^{+\infty} \int_{-\infty}^{0}\left|\frac{\partial \psi}{\partial x}\right|^{2} d X d Y-\omega^{2} \int_{0}^{+\infty} \psi^{2}(X, 0) d X
$$

Now, for every $u \in H^{1}(]-\infty, 0[)$ we have

$$
u(0)^{2}=\int_{-\infty}^{0} \frac{d}{d y}\left(u^{2}\right) d y=2 \int_{-\infty}^{0} u(y) \frac{d u}{d y}(y) d y
$$

and therefore, $\forall \epsilon>0$ :

$$
u(0)^{2} \leq \epsilon^{2} \int_{-\infty}^{0} u^{2} d y+\frac{1}{\varepsilon^{2}} \int_{-\infty}^{0}\left|\frac{d u}{d y}\right|^{2} d y
$$

From (5.30) and (5.31), by choosing $\varepsilon=\frac{\omega}{\sin \alpha}$, we derive finally (5.29).

Let us now denote by $a_{\alpha}(\omega ; \varphi, \varphi)$ the bilinear from associated to $A_{\alpha}(\omega)$ and by $S_{m}^{\prime \alpha}(\omega)$ the sequence of Max-Min $(m \geq 1)$

$$
S_{m}^{\alpha}(\omega)=\inf _{V \in \mathcal{V}_{m}\left(\Omega_{\alpha}\right)} \sup _{\varphi \in V, \varphi \neq 0} \frac{a_{\alpha}(\omega ; \varphi, \varphi)}{|\varphi|_{\Omega_{\alpha}}^{2}}
$$

Notice that $\Omega_{\alpha}$ is invariant under every scaling of the form

$$
X=\tau x, Y=\tau y, \tau>0
$$

so that we have, trivially

$$
S_{m}^{\alpha}(\tau \omega)=\tau^{4} S_{m}^{\alpha}(\omega)
$$

In the following, we set

$$
S_{m}^{\alpha}=S_{m}^{\alpha}(1)
$$

so that

$$
S_{m}^{\alpha}(\omega)=\omega^{4} S_{m}^{\alpha}
$$

To complete the general spectral theory of $A_{\alpha}(\omega)$, we will now establish the 
Lemma $5.11 \sigma_{e . s s}\left(A_{c, r}(\omega)\right)=\left[-\omega^{4},+\infty[\right.$.

Proof : First we prove the inclusion : $\sigma_{e s s}\left(A_{x,}(\omega)\right) \subset\left[-\omega^{4},+\infty\right]$

By the Min-Max principle : Inff $\sigma_{c s s}\left(A_{\alpha}(\omega)\right)=\lim _{n \rightarrow+\infty} S_{m}^{c x}(\omega)$. Consider now the profile $h_{i, D}$ defined by :

$$
h_{\alpha, D}(x)= \begin{cases}h_{\alpha}(x)=x \tan \alpha & \text { if } x<D \\ D \tan \alpha & \text { if } x>D\end{cases}
$$

and by $S_{m}^{\alpha, D}(\omega)$ the associated Min-Max. Then we have clearly :

$$
S_{m}^{\alpha, D}(\omega) \leq S_{m}^{\alpha}(\omega) \quad \forall \omega>0 \quad \forall D>0
$$

and by theorem 4.4 .

$$
\lim S_{m}^{\alpha, D}(\omega)=-\beta_{D}^{2}(\omega)
$$

where $\beta_{D}(\omega)$ satifies : $\quad \beta_{D}(\omega) \tanh \left(D \beta_{D}(\omega)\right)=-\omega^{2}$.

Consequently :

$$
\lim _{m \rightarrow+\infty} S_{m}^{\alpha}(\omega) \geq \limsup _{D \rightarrow+\infty}\left(-\beta_{D}^{2}(\omega)\right) \geq-\omega^{2}
$$

To prove the converse inclusion, we consider for every $\sigma<\omega^{4}$ the singular sequence:

$$
\psi_{n}(x, y)=\frac{C(\alpha) \beta}{\sqrt{n}} O\left(\frac{x}{n}\right) \eta\left(\frac{y}{n}\right) e^{\omega^{2} y} e^{i \sqrt{\omega^{4}-\sigma x}}
$$

where $O(x)$ and $\eta(y)$ are $C^{\infty}$ cut-off functions with values between 0 and 1 such that

$$
\begin{gathered}
\left\{\begin{array}{cl}
\theta(x)=1 & \text { if } 1.5<x<2.5 \\
\theta(x)=0 & \text { if } x<1 \text { or } x>3
\end{array}\right. \\
\vdots \\
\begin{cases}\eta(y)=1 & \text { if } \frac{-\tan (\alpha)}{2}<y<0 \\
\eta(y)=0 & \text { if } y<-\tan (\alpha)\end{cases}
\end{gathered}
$$

and $C(\alpha)$ is an appropriate normalisation constant. We conclude as in the proof of theorem 4.3 .

In the following, we will denote by $M(\alpha)$ the number (independent on $\omega$ ) of eigenvalues of $A_{\alpha}(\omega)$ located below the essential spectrum

$$
M(\alpha)=\sup \left\{m \in N^{*} ; S_{m}^{\alpha}<-1\right\}
$$

Ursell $(\{30\})$ proved that

$$
M(\alpha) \geq \operatorname{Max}\left\{m \in \mathbf{N} ;(2 m-1) \alpha<\frac{\pi}{2}\right\}
$$


(This inequality is probably an equality but we do not know any proof of this result).

We can now establish the main result of this section :

Theorem 5.6 If the depth function hatisfies (5.27), then:

$$
\limsup _{\omega \rightarrow+\infty} \frac{S_{m}(\omega)}{\omega^{4}} \leq S_{m}^{\alpha} \text { for } m=1,2 \ldots M(\alpha)
$$

and $N(\omega) \geq M(\alpha)$ for large $\omega$, where $M(\alpha)$ is defined by (5.34).

Proof: 1 - Notice first that we just have to establish (5.36). Indeed, if (5.36) holds, then

$$
S_{m}(\omega)+\beta_{\infty}^{2}(\omega)<\omega^{4}\left(S_{n}^{\alpha}+\frac{\beta_{\infty}^{2}(\omega)}{\omega^{4}}\right)
$$

for large $\omega$ and for $m=1,2 \ldots M(\alpha)$.

Moreover, by definition of $M(\alpha)$ :

$$
S_{m}(\alpha)<-1 \text { for } m=1,2 \ldots M(\alpha)
$$

and by $(2.2)$ :

$$
\lim _{\omega \rightarrow+\infty} \frac{\beta_{\infty}^{2}(\omega)}{\omega^{4}}=1
$$

Consequently, for $m=1,2 \ldots M(\alpha)$

$$
S_{m_{2}}(\omega)<-\beta_{\infty}^{2}(\omega) \text {, for large } \omega .
$$

2 - We will now prove (5.36).

For $m=1,2 \ldots M(\alpha)$, we denote by $\psi_{m}$ an eigenfunction of $A_{\alpha}(1)$ associated to the eigenvalue $S_{m}^{\alpha}$ and such that :

$$
\int_{\Omega_{a}} \psi_{m} \psi_{m} d x d y=\delta_{m n}
$$

Notice that $\tilde{\psi}_{m}(\omega ; x, y)=\psi_{m}\left(\omega^{2} x, \omega^{2} y\right)$ is an eigenfunction of $A_{\alpha}(\omega)$ associated to the eigenvalue $S_{m}^{\alpha}(\omega)$.

To establish (5.36), we would like to use the functions $\bar{\psi}_{m}(\omega)$ as test functions in formula (4.21). But $\bar{\psi}_{m}(\omega)$ is defined in $\Omega_{\alpha}$ and hypothesis (5.27) does not ensure generally that $\Omega \subset \Omega_{\alpha}$ (see figure 13). For that reason we define the extended functions $\dot{\varphi}_{m}(\omega)$ on $\Omega$ as follows

$$
\tilde{\varphi}_{m}(\omega ; x, y)=\left\{\begin{array}{l}
\tilde{\psi}_{m}(\omega ; x, y) \text { if } \theta \leq \alpha \\
\bar{\psi}_{m}(\omega ; r \cos \alpha, r \sin \alpha) \text { if } \alpha<0<\frac{\pi}{2}
\end{array}\right.
$$


where $r$ and $\theta$ are the polar coordinates associated to $x$ and $y$. We set also : $\varphi_{m}(x, y)=$ $\tilde{\varphi}_{n}(1 ; x, y)$.

If $V_{m}(\omega)$ denotes the m-dimensional subspace of $H^{1}(\Omega)$ spanned by $\tilde{\varphi}_{1}(\omega), \ldots \tilde{\varphi}_{m}(\omega)$, we obtain by (4.21)

$$
S_{m}(\omega) \leq \sup _{\dot{\varphi} \in V_{m}(\omega), \tilde{\varphi} \neq 0} \frac{a(\omega ; \tilde{\varphi}, \tilde{\varphi})}{|\tilde{\varphi}|_{\Omega}^{2}}
$$

After the change of coordinates $X=\omega^{2} x, Y=\omega^{2} y$, this inequality becomes :

$$
S_{m}(\omega) \leq \omega^{4} \sup _{\varphi \in V_{m}, \varphi \neq 0} \frac{\int \chi(\omega)|\nabla \varphi|^{2} d X d Y-\int_{0}^{+\infty} \varphi^{2}(X, 0) d X}{\int \chi(\omega) \varphi^{2} d X d Y}
$$

where $V_{m}$ is the space spanned by $\varphi_{1}, \varphi_{2} \ldots \varphi_{m}$ and $\chi(\omega)$ is defined as follows:

$$
\chi(\omega)=\left\{\begin{array}{cc}
1 & \text { if }-\omega^{2} h\left(\frac{X}{\omega^{2}}\right)<Y<0 \\
0 & \text { elsewhere }
\end{array}\right.
$$

3 - We will achieve the proof by contradiction. Suppose there exists $\epsilon>0$, a sequence $\xi_{p} \in V_{m}$ and a sequence $\omega_{p} \rightarrow \infty$ such that :

$$
\left\{\begin{array}{l}
\int \chi\left(\omega_{p}\right)\left|\nabla \xi_{p}\right|^{2} d X d Y-\int_{0}^{+\infty} \xi_{p}^{2}(X, 0) d X \\
\geq\left(S_{m}^{\alpha}+\epsilon\right) \int \chi\left(\omega_{p}\right) \xi_{p}^{2} d X d Y
\end{array}\right.
$$

This sequence can be normalized as follows

$$
\int_{\Omega_{\alpha}} \xi_{p}^{2} d x d y=1
$$

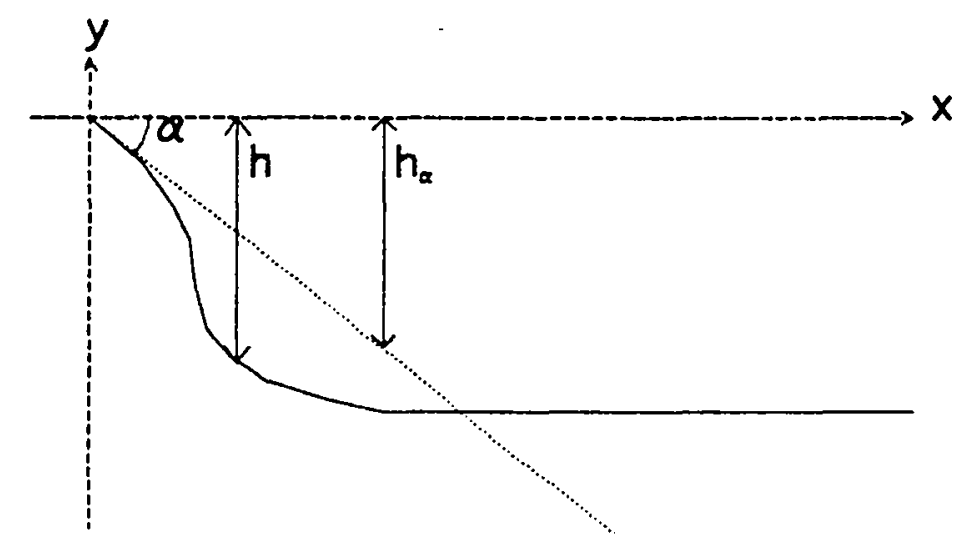

Figure 13: The domains $\Omega$ and $\Omega_{\alpha}$ 
Since $V_{n}$ is finite-dimensional, $\left(\xi_{p}\right)$ has a convergent subsequence which we denote also by $\left(\xi_{p}\right)$;

$$
\xi_{p} \rightarrow \xi \text { in } V_{m}
$$

By theorem of dominated convergence of Lebesgue we obtain

$$
\left\{\begin{array}{l}
\int_{\Omega_{\alpha}}|\nabla \xi|^{2} d X d Y-\int_{0}^{+\infty} \xi^{2}(X, 0) d X \geq S_{m}^{\alpha}+\epsilon \\
\int_{\Omega_{\alpha}} \xi^{2} d X d Y=1
\end{array}\right.
$$

since $\chi(\omega)$ tends, when $\omega$ tends to infinity, to the characteristic funtion of $\Omega_{\alpha}$. But this is impossible by definition of $V_{m}$ and $S_{m}^{\alpha}$.

Since (5.38) cannot occur, (5.39) implies (5.36).

A straightforward consequence of theorem 5.6 is the

Corollary 5.5 If the depth function h satifies (5.27), then

$$
\limsup _{\omega \rightarrow+\infty} \frac{-S_{m}(\omega)-\beta_{\infty}^{2}(\omega)}{\omega^{4}} \geq S_{m}^{\alpha}+1
$$

for $m=1,2 \ldots M(\alpha)$.

This means that the potential $\varphi_{m}(\omega)$ associated to $S_{m}(\omega)$ becomes more and more confined when the frequency increases.

Suppose now that $h$ satisfies (5.27) with $\alpha \geq \frac{\pi}{4}$. Then, by (5.35) and by theorem 5.6 , there is at least one guided mode at high frequency.

We will prove now some converse results.

Theorem 5.7 Suppose that the depth profile $h$ satisfies

$$
h(x) \geq \operatorname{Min}\left(x \tan \frac{\pi}{4}, h_{\infty}\right) .
$$

Then

$$
S_{2}(\omega)=-\beta_{\infty}^{2}(\omega), \quad \forall \omega
$$

and therefore

$$
N(\omega) \leq 1, \quad \forall \omega .
$$

Proof: By the comparison principle, we just have to establish the theorem for

$$
h(x)=\operatorname{Min}\left(x \tan \frac{\pi}{4}, h_{\infty}\right)
$$

We set (cf. figure 14)

$$
\Omega_{i}=\left\{(x, y) \in \Omega ; x<h_{\infty}\right\}
$$




$$
\Omega_{\odot}=\left\{(x, y) \in \Omega ; x>h_{\infty}\right\}
$$

Then by lemma 5.6

$$
S_{2}(\omega) \geq \operatorname{Min}\left(S_{2}^{N}(\omega),-\beta_{\infty}^{2}(\omega)\right) \quad \forall \omega
$$

But we can calculate explicitly the eigenvalues $S_{m}^{N}(\omega)$ (cf. annex) and we obtain :

$$
\left\{\begin{array}{l}
S_{1}^{N}(\omega)=-2 \beta_{\omega}^{2}(\omega) \\
S_{2}^{N}(\omega)=-\beta_{\infty}^{2}(\omega)+\nu_{1}^{2}(\omega)
\end{array}\right.
$$

where $\nu_{1}(\omega)$ is the smallest positive solution $\nu$ of :

$$
\nu \tan \left(\nu h_{\infty}\right)=-\omega^{2}
$$

That proves the theorem.

More generally, we have the

Theorem 5.8 Suppose that the depth function h satifies

$$
h(x) \geq x \tan \frac{\pi}{4} \text { for } 0<x<h_{*}
$$

then

$$
S_{3}(\omega)=-\beta_{\infty}^{2}(\omega) \text { for large } \omega
$$

and therefore:

$$
N(\omega) \leq 2 \text { for large } \omega .
$$

Proof: As previously, we just have to consider the case where

$$
h(x)=x \tan \frac{\pi}{4} \text { for } 0<x<h_{*}
$$

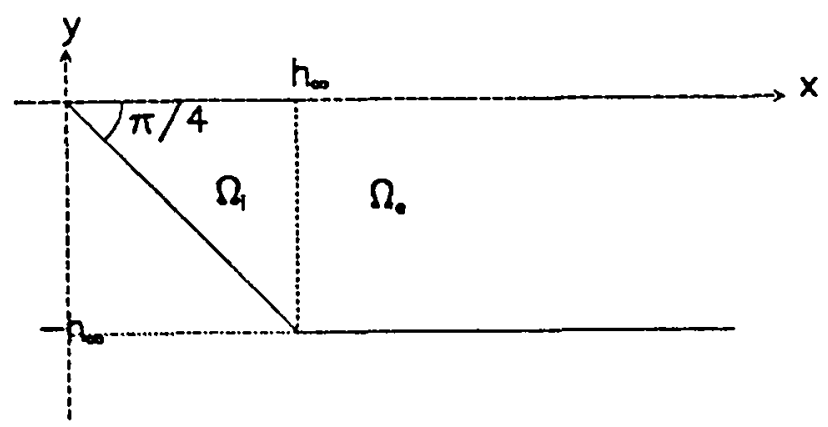

Figure 14: The domains $\Omega_{i}$ and $\Omega_{e}$ 
and we set (cf figure 15):

$$
\begin{aligned}
& \Omega_{i}=\left\{(x, y) \in \Omega ; x<h_{*}\right\} \\
& \Omega_{e}=\left\{(x, y) \in \Omega ; x>h_{*}\right\} \\
& h_{*}^{-}=\operatorname{Inf}\left\{h(x), x>h_{*}\right\}
\end{aligned}
$$

We suppose that $h_{*}^{-}>h_{\infty}$ otherwise the previous proof would work.

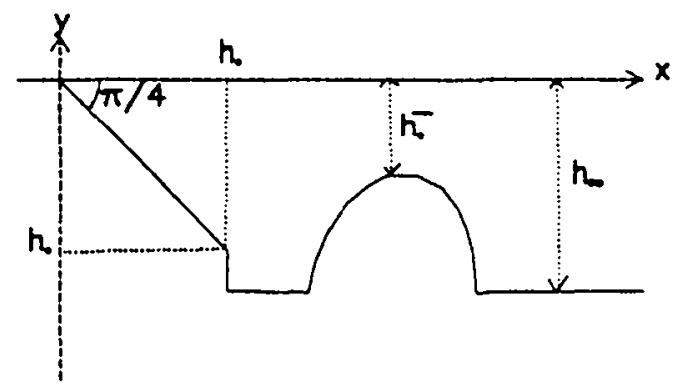

Figure 15: Illustration of theorem 5.8

Denote, with obvious notations, by $S_{m}^{N, i}(\omega)$ and $S_{m}^{N, e}(\omega)$ the min-max associated to the domains $\Omega_{i}$ and $\Omega_{e}$.

By theorems 5.1 and 5.5, we have

$$
S_{1}^{N, e}(\omega)<-\beta_{\infty}^{2}(\omega)=S_{2}^{N, e}(\omega) \text { for large } \omega
$$

and likewise (cf.annex):

$$
S_{1}^{N, i}(\omega)<-\beta_{\infty}^{2}(\omega)<S_{2}^{N, i}(\omega) \text { for large } \omega
$$

Let us denote by $\varphi_{i}(\omega)$ the eigenfunction associated to $S_{1}^{N, i}$ and by $\varphi_{e}(\omega)$ the one associated to $S_{1}^{N, e}(\omega)$. Then by $(4.20)$ :

$$
S_{3}(\omega) \geq \inf _{\varphi \in W ; \varphi \neq 0} \frac{a(\omega ; \varphi, \varphi)}{|\varphi|_{\Omega}^{2}} \text { for large } \omega
$$

where

$$
W=\left\{\varphi \in H^{1}(\Omega) ; \int_{\Omega_{i}} \varphi \varphi_{i}(\omega) d x d y=\int_{\Omega e} \varphi \varphi_{e}(\omega) d x d y=0\right\}
$$

But clearly, for $\varphi \in W$

and therefore

$$
\left\{\begin{array}{l}
a_{i}(\omega ; \varphi, \varphi) \geq-\beta_{\infty}^{2}(\omega)|\varphi|_{\Omega_{\ell}}^{2} \\
a_{e}(\omega ; \varphi, \varphi) \geq-\beta_{\infty}^{2}(\omega)|\varphi|_{\Omega_{e}}^{2}
\end{array}\right.
$$

$$
a(\omega ; \varphi, \varphi) \geq-\beta_{\infty}^{2}(\omega)|\varphi|_{\Omega}^{2} \text { for large } \omega .
$$

Remark 5.5 To establish theorems 5.7 and 5.8, we use the explicit expressions of the eigenvalues for a triangle with angle $\frac{\pi}{4}$ wich are presented in annex. That is the reason why we did not succeed to extend these results to angles $\alpha<\frac{\pi}{4}$. 


\subsection{The case of the tangential beach}

Our goal in this paragraph is to establish some theorems proving that for the type of geometry we denoted by "tangential beach" in section 2 (see figure 5), the number of trapping waves tends to infinity when the frequency increases to infinity. For technical reasons, we did not succeed to establish rigorously such a result for the general assumption that $h(0)=h^{\prime}(0)=0$. We shall restrict ourselves to state a theorem corresponding to stronger, while any way not really restrictive, assumptions on the geometry of the coast. Our result is the following

Theorem 5.9 Assume that there exists three strictly positive numbers $\left(\alpha, A, x_{0}\right)$ such that

$$
\forall x \in\left[0, x_{0}\right], \quad h(x) \leq A x^{1+\alpha}
$$

Then there exists an increasing sequence of positive numbers $\omega_{m}, m \geq 1$ such that for $\omega \geq$ $\omega_{m}, A(\omega)$ has at least $m$ eigenvalues in its discrete spectrum. Moreover the sequence $\omega_{m}$ necessarily tends to infinity.

Proof: We give here a direct proof. Thanks to the comparison result of lemma 5.3, we can restrict ourselves to consider the case where $h(x)$ satisfies :

$$
h(x)=A x^{1+\alpha} \quad x \in\left[0, x_{0}\right]
$$

Once again, thanks to the Min-Max principle, the game consists in finding an $\mathrm{m}$-dimensional subspace $V_{m}$ of $H^{1}(\Omega)$ such that

$$
a(\omega ; \phi, \phi)<-\beta_{\infty}(\omega)^{2}|\phi|^{2} \quad \forall \phi \in V_{m}
$$

If this holds, we know that the discrete spectrum of $A(\omega)$ contains at least $\mathrm{m}$ eigenvalues. Let us consider particular test functions in the form

$$
\phi(x, y)=\frac{\cosh \left(\beta_{\infty}(\omega)(y+h(x))\right)}{\cosh \left(\beta_{\infty}(\omega) h(x)\right)} \varphi(x) .
$$

where the function $\varphi(x)$ satifies

$$
\varphi \in H^{1}\left(\mathbb{R}^{+}\right) ; \operatorname{supp} \varphi \subset\left[0, x_{0}\right]
$$

By an explicit calculation, it is easy to obtain the following identity (we simply set $\beta=$ $\beta_{\infty}(\omega)$ to simplify the notation )

$$
\begin{aligned}
a(\omega ; \phi, \phi)+\beta^{2}|\phi|^{2} & =\beta \int_{0}^{x_{0}}\left[\tanh \beta h(x)-\tanh \beta h_{\infty}\right] \varphi(x)^{2} d x \\
& +\int_{0}^{x_{0}} \int_{-h(x)}^{0}\left|\frac{\partial \phi}{\partial x}(x, y)\right|^{2} d x d y
\end{aligned}
$$


where $\frac{\partial \phi}{\partial t}$ is given by

$$
\frac{\partial \phi}{\partial x}(x, y)=\frac{\cosh (\beta(y+h(x)))}{\cosh ^{2} \beta h(x)} \varphi^{\prime}(x)+\beta \frac{\sinh \beta y}{\cosh ^{2} \beta h(x)} h^{\prime}(x) \varphi(x)
$$

(we have used the fact that $h(x)$ is differentiable in the interval $\left[0, x_{0}\right]$ ). Let us now estimate the two terms of the right hand side of (5.45). From the double inequality

$$
1-2 e^{-2 x} \leq \tanh x \leq 1-e^{-2 x}, x>0
$$

we deduce that

$$
\tanh \beta h(x)-\tanh \beta_{\infty} \leq 2 e^{-2 \beta h_{\infty}}-e^{-2 \beta h(x)}
$$

so that

$$
\begin{aligned}
\int_{0}^{x_{0}}\left\{\tanh \beta h(x)-\tanh \beta h_{\infty}\right\} \varphi(x)^{2} d x & \leq 2 e^{-2 \beta h_{\infty}} \int_{0}^{x_{0}} \varphi(x)^{2} d x \\
& -\int_{0}^{x_{0}} e^{-2 \beta h(x)} \varphi(x)^{2} d x
\end{aligned}
$$

Using the three inequalities :

$$
\mid \begin{aligned}
& \left|\frac{\partial \phi}{\partial x}(x, y)\right|^{2} \leq 2 \varphi^{\prime}(x)^{2} \frac{\cosh ^{2} \beta(y+h(x))}{\cosh ^{2} \beta h(x)}+2 \beta^{2} h^{\prime}(x)^{2} \frac{\sinh ^{2} \beta y}{\cosh ^{4} \beta h(x)} \varphi(x)^{2} \\
& \int_{-h(x)}^{0} \cosh ^{2} \beta(y+h(x)) d y \leq \frac{\sinh 2 \beta h(x)}{2 \beta} \\
& \int_{-h(x)}^{0} \sinh ^{2} \beta y d y \leq \frac{\sinh 2 \beta h(x)}{4 \beta}
\end{aligned}
$$

we easily obtain that :

$$
\begin{aligned}
\int_{0}^{x_{0}} \int_{h-(x)}^{0}\left|\frac{\partial \phi}{\partial x}(x, y)\right|^{2} d x d y & \leq \frac{2}{\beta} \int_{0}^{x_{0}} \tanh \beta h(x) \varphi^{\prime}(x)^{2} d x \\
& +\beta \int_{0}^{x_{0}} \frac{\tanh \beta h(x)}{\cosh ^{2} \beta h(x)} h^{\prime}(x)^{2} \varphi(x)^{2} d x
\end{aligned}
$$

As for $z>0$ we have $\tanh z<1$ and $\frac{\tanh z}{\cosh ^{2} z}<4 e^{-2 z}$, we have finally shown that :

$$
a(\omega ; \phi, \phi)+\beta^{2}|\phi|^{2} \leq a_{1}(\omega ; \varphi, \varphi)-a_{0}(\omega ; \varphi, \varphi)
$$

for any test function $\phi$ in the form (5.44), where the two quadratic forms $a_{1}(\omega ; . ;$.$) and$ $a_{0}(\omega ; .,$.$) are defined in H^{1}\left(\boldsymbol{R}^{+}\right)$by :

$$
\mid \begin{aligned}
a_{1}(\omega ; \varphi, \varphi) & =\frac{2}{\beta} \int_{0}^{x_{0}} \varphi^{\prime}(x)^{2} d x+4 \beta \int_{0}^{x_{0}} e^{-2 \beta h(x)} h^{\prime}(x)^{2} \varphi(x)^{2} d x \\
& +2 \beta e^{-2 \beta h_{\infty}} \int_{0}^{x_{0}} \varphi(x)^{2} d x \\
a_{0}(\omega ; \varphi, \varphi) & =\beta \int_{0}^{x_{0}} e^{-2 \beta h(x)} \varphi(x)^{2} d x
\end{aligned}
$$


We now use the particular form of $h(x)$ given by (5.14). In this case, we have :

$$
\mid \begin{aligned}
a_{1}(\omega ; \varphi, \varphi) & =\frac{2}{\beta} \int_{0}^{x_{0}} \varphi^{\prime}(x)^{2} d x+4 \beta A^{2}(1+\alpha)^{2} \int_{0}^{x_{0}} x^{2 \alpha} e^{-2 A \beta x^{1+\alpha}} \varphi(x)^{2} d x \\
& +2 \beta e^{-2 \beta h_{\infty}} \int_{0}^{x_{0}} \varphi(x)^{2} d x \\
a_{0}(\omega ; \varphi, \varphi) & =\beta \int_{0}^{x_{0}} e^{-2 A \beta x^{1+\alpha}} \varphi(x)^{2} d x
\end{aligned}
$$

Now let us choose our function $\varphi(x)$ in the form

$$
\varphi(x)=\psi\left(\beta^{\frac{1}{1+\alpha}} x\right)
$$

where $\psi$ is compactly supported is some interval $[0, \mathrm{~K}]$ so that, at least for $\omega$ large enough, $\varphi$ is compactly supported in the interval $\left[0, x_{0}\right]$. For such a function $\varphi$, a change of variable $y=\beta^{\frac{1}{1+\alpha}} x$ permits us to establish the following equalities:

$$
\mid \begin{aligned}
a_{1}(\omega ; \varphi, \varphi) & =2 \beta^{-\frac{\alpha}{3+\alpha}} \int_{0}^{+\infty} \psi^{\prime}(y)^{2} d y+4(1+\alpha)^{2} A^{2} \beta^{-\frac{\alpha}{1+\alpha}} \int_{0}^{+\infty} y^{2 \alpha} e^{-2 A y^{1+\alpha}} \psi(y)^{2} d y \\
& +2 e^{-2 \beta h_{\infty} \beta^{\frac{\alpha}{1+\alpha}}} \int_{0}^{+\infty} e^{-2 y^{1+\alpha}} \psi(y)^{2} d y \\
a_{0}(\omega ; \varphi, \varphi) & =\beta^{\frac{\alpha}{1+\alpha}} \int_{0}^{+\infty} e^{2 A y^{1+\alpha}} \psi(y)^{2} d y
\end{aligned}
$$

Consequently, there exist two positive constants $C_{1}=C_{1}\left(\alpha, A, h_{\infty}\right)$ and $C_{2}=C_{2}(\alpha, A, K)$ independent on $\omega$ such that, for $\omega$ large enough :

$$
\mid \begin{gathered}
a(\omega ; \phi, \phi)+\beta_{\infty}(\omega)^{2}|\varphi|^{2} \leq \quad C_{1} \beta_{\infty}(\omega)^{-\frac{\alpha}{1+\alpha}} \int_{0}^{+\infty}\left(\psi^{\prime}\left(y^{2}\right)+\psi(y)^{2}\right) d y \\
-C_{2} \beta_{\infty}(\omega)^{\frac{\alpha}{1+\alpha}} \int_{0}^{+\infty} \psi(y)^{2} d y
\end{gathered}
$$

Now, let $U_{n}$ be a m-dimensional subspace of functions in $H^{1}[0,+\infty]$ compactly supported in the interval $\left[0, K^{\prime}\right]$. For instance, take $U_{m}$ defined by :

$$
U_{m}=\operatorname{span}\left[w_{1}(y), \ldots, w_{m}(y)\right\}
$$

where $w_{j}(y)=\sin \frac{j \pi y}{K}$ for $y \in[0, K]$ and $w_{j}(y)=0$ for $y>K$. We know that, for any $\psi$ in the space $U_{m}$

$$
\int_{0}^{+\infty} \psi^{\prime}(y)^{2} d y \leq \frac{m^{2} \pi^{2}}{K^{2}} \int_{0}^{+\infty} \psi(y)^{2} d y
$$

Now, let us denote by $V_{m}(\omega)$ the m-dimensional subspace of $H^{1}(\Omega)$ defined by :

$$
\phi \in V_{m}(\omega) \Longleftrightarrow\left\{\begin{array}{l}
\phi(x, y)=\frac{\cosh \beta_{\infty}(\omega)(y+h(x))}{\cosh \beta_{\infty}(\omega) h(x)} \varphi(x) \\
\varphi(x)=\psi\left(\beta_{\infty}(\omega)^{\frac{1}{1+\alpha}} x\right), \psi \in U_{m}
\end{array}\right.
$$


Thanks to (5.48) and (5.49), we obtain for any $\phi$ in $V_{m}(\omega)$ :

$$
a(\omega ; \phi, \phi)+\beta_{\infty}(\omega)^{2}|\varphi|^{2} \leq\left\{C^{1} \beta_{\infty}(\omega)^{-\frac{\alpha}{1+\alpha}}\left(1+\frac{m^{2} \pi^{2}}{K^{2}}\right)_{-} C^{2} \beta_{\infty}(\omega)^{\frac{\alpha}{1+\alpha}}\right\} \int_{0}^{+\infty} \psi(y)^{2} d y
$$

It suffices to take $\omega$ large enough such that

$$
\beta_{\infty}(\omega)^{\frac{2 \alpha}{1+\alpha}}>\frac{C_{1}}{C_{2}}\left(1+\frac{m^{2} \pi^{2}}{K^{2}}\right)
$$

which is equivalent to some inequality $\omega>\omega_{m}$ where $\omega_{m}$ depends on $m, \alpha, h_{\infty}, \mathrm{K}$ and $\mathrm{A}$, to get :

$$
\forall \phi \in V_{m}(\omega) \quad \omega>\omega_{m} \Rightarrow a(\omega ; \phi, \phi)+\beta_{\infty}(\omega)^{2}|\phi|^{2}<0
$$

which means that for $\omega>\omega_{m}, A(\omega)$ has at least $\mathrm{m}$ eigenvalues. Note that the sequence $\omega_{m}$ tends to $+\infty$ This could have been guessed as a consequence of the fact that the number $N(\omega)$ of eigenvalues of $A(\omega)$ smaller than $-\beta_{\infty}(\omega)^{2}$ is finite for any $\omega$ (cf. theorem 4.5). By contradiction, one easily proves that $\omega_{m}$ cannot accumulate to some finite value.

Let us notice that :

1 - One can give another proof of theorem 5.9 only based on the use of comparison results.

2 - In fact, one can introduce the upper thresholds as the numbers $\left\{\omega_{m}^{*}, m \in N\right\}$ which are defined by :

$$
\omega_{m}^{*}=\inf \left\{\omega_{m} / \forall \omega \geq \omega_{m}, A(\omega) \text { has at least } m \text { eigenvalues in its discrete spectrum }\right\}
$$

and the lower thresholds $\omega_{m}^{\circ}, m \in \mathbf{N}^{*}$ defined by

$$
\omega_{m}^{c}=\sup \left\{\omega_{m} / \forall \omega<\omega_{m}, A(\omega) \text { has at most } \mathrm{m}-1 \text { eigenvalues in its discrete spectrum }\right\}
$$

Then, under assumption (5.42), it is clear that one has the following properties

$$
\mid \begin{array}{ll}
\text { (i) } \quad \lim _{m \rightarrow+\infty} \omega_{m}^{o}=+\infty \\
\text { (ii) } \quad \forall m \geq 1 \quad \omega_{m}^{\circ} \leq \omega_{m}^{*} \\
\text { (iii) } \quad \forall m \geq 1 \quad \omega_{m}^{\circ}<+\infty
\end{array}
$$

In fact properties (ii), (iii) are general while (i) is specific to the case described by assumption (5.42). This situation is analogous to the one encountered in more classical waveguides problems as in acoustics [34], optical fibers ([1] and [4]) or elastodynamics ([2] and [3]). 3 - The main idea of the proof is the use of test functions $\varphi$ in the form (5.47) where $\phi$ is of the form (5.44). This choice corresponds to a scaling in the $x$-direction adapted to the way $h(x)$ tends to zero when $x$ tends to 0 . The test functions concentrate more and more near $x=0$ as $\omega$ tends to $+\infty$ and this phenomenon is more and more pronounced as $\alpha$ increases. 
4 - One can give some upper bounds for the upper thresholds $\omega_{m}^{*}$. Indeed, by (5.50), $\omega_{m}^{*} \leq \omega_{m}$ where $\omega_{m}$ is defined by :

$$
\omega_{m}=\beta_{n} \tanh \left(\beta_{m} h_{\infty}\right) \quad \text { with } \quad \beta_{m}=\left[\frac{C_{1}}{C_{2}}\left(1+\frac{m^{2} \pi^{2}}{K^{2}}\right)\right]^{\frac{1+\alpha}{2 x}}
$$

In this estimate as in the proof oh the theorem, $\mathrm{K}$ appears as an arbitrary parameter. A simple calculation shows that the value of $\omega_{m}$ is minimized for $K=K(m)$ where :

$$
\lim _{m \rightarrow+\infty} K(m)=[A(1+\alpha)]^{-\frac{1}{1+\alpha}}
$$

In other words, we have proved that the upper thresholds $\omega_{m}^{*}$ satisfy :

$$
\omega_{m}^{*} \leq \omega_{m}
$$

where, for large $\mathrm{m}$,

$$
\omega_{m} \sim C m^{\frac{1+\alpha}{2 \alpha}}
$$

where the constant $\mathrm{C}$ only depends on $\mathrm{A}, \alpha$ and $h_{\infty}$

This result means that, a priori, when $\alpha$ increases i.e. when the beach is more and more tangent, the new guided waves appear sooner and sooner with respect to the frequency. Note also that as $\frac{1+\alpha}{2 \alpha} \longrightarrow+\infty$ when $\alpha$ tends to 0 , such an estimate is coherent with the fact that, for $\alpha=0$ the number of guided modes is uniformly bounded with respect to the frequency $\omega$. 


\section{The numerical approach}

In this section, we describe a numerical method for determining the guided waves for an arbitrary relief. Some results of computation are then presented, to illustrate the previous theoretical ones.

\subsection{The numerical method}

\subsubsection{The discretization}

The formulation which is the most appropriate for numerical purposes has been described in section 3.2. For any given pulsation $\omega$, the guided modes correspond to the couples $(\varphi, \lambda)$ such that :

$$
\left\{\begin{array}{l}
\lambda \in \mathbb{R}, \lambda<-\beta_{\infty}^{2}(\omega) \\
\varphi \in D(B(\omega, \lambda)), \varphi \neq 0 \\
B(\omega, \lambda) \varphi=\lambda \varphi
\end{array}\right.
$$

where the operator $B(\omega, \lambda)$ is defined by (3.13).

Problem (6.1) can be written in the following variational form :

$$
\left\{\begin{array}{l}
\text { Find } \lambda \in \mathbb{R}, \lambda<-\beta_{\infty}^{2}(\omega), \text { and } \varphi \in H^{1}\left(\Omega_{i}\right), \varphi \neq 0, \text { such that : } \\
\forall \psi \in H^{1}\left(\Omega_{i}\right), b(\omega, \lambda ; \varphi, \psi)=\lambda \int_{\Omega_{i}} \varphi \psi d x d y,
\end{array}\right.
$$

where $b(\omega, \lambda ; \varphi, \psi)$ is defined by (4.5).

The great advantage of this formulation is that it is set in a bounded domain and can therefore be discretized by standard finite elements.

Let us denote by $V_{h}^{\prime}$ a finite elements space such that $V_{h} \subset H^{1}\left(\Omega_{i}\right)$ and consider the following problem :

$$
\left\{\begin{array}{l}
\text { Find } \lambda \in \mathbb{R}, \lambda<-\beta_{\infty}^{2}(\omega), \text { and } \varphi_{h} \in V_{h}, \varphi_{h} \neq 0, \text { such that : } \\
\forall \psi_{h} \in V_{h}, b_{N}\left(\omega, \lambda ; \varphi_{h}, \psi_{h}\right)=\lambda \int_{\Omega_{i}} \varphi_{h} \psi_{h} d x d y
\end{array}\right.
$$

Here, $b_{N}(\omega, \lambda ; \varphi, \psi)$ denotes an approximation of $\mathrm{b}(\omega, \lambda ; \varphi, \psi)$ defined as follows :

$$
b_{N}(\omega, \lambda ; \varphi, \psi)=\int_{\Omega_{i}} \nabla \varphi \cdot \nabla \psi d x d y-\omega^{2} \int_{\Gamma_{F}^{i}} \varphi \psi d \sigma+\left\langle T_{N}(\omega, \lambda) \varphi, \psi\right\rangle_{\Sigma}
$$

where

$$
\left\langle T_{N}(\omega, \lambda) \varphi, \psi\right\rangle_{\Sigma}=\sum_{n=0}^{N} \alpha_{n}(\lambda, \omega)\left\langle\varphi, u_{n}(\omega)\right\rangle_{\Sigma}\left\langle\psi, u_{n}(\omega)\right\rangle_{\Sigma}
$$

whith the notations of lemma (3.2). 
The convergence of problem (6.3) when $h$ tends to 0 and $N$ to infinity has been studied for other problems (see [4], [9], [17] and [22]). This point will not be studied here since our purpose is just to illustrate the previous theoretical results.

\subsubsection{A non-linear eigenvalue problem}

Suppose $\left(\xi_{j}\right)_{j=1, P}$ is a basis of $V_{h}$. Then problem (6.3) can be written, using matrices, in the following form:

$$
\left\{\begin{array}{l}
\text { Find } \lambda \in \mathbb{R}, \lambda<-\beta_{\infty}^{2}(\omega), \text { and } U \in \mathbb{R}^{P}, U \neq 0, \text { such that : } \\
\mathbf{B}_{h, N}(\omega, \lambda) U=\lambda \mathbf{M}_{h} U
\end{array}\right.
$$

where the matrices $\mathbf{B}_{h, N}(\omega, \lambda)$ and $\mathbf{M}_{h}$ are defined by :

$$
\left\{\begin{array}{l}
{\left[\mathbf{B}_{h, N}(\omega, \lambda)\right]_{i, j}=b_{N}\left(\omega, \lambda ; \xi_{h}^{(j)}, \xi_{h}^{(k)}\right)} \\
{\left[\mathbf{M}_{h}\right]_{j, k}=\int_{\Omega_{i}} \xi_{h}^{(j)} \xi_{h}^{(k)} d x d y}
\end{array}\right.
$$

Problem (6.6) is a "non-linear" generalized eigenvalue problem, in the sense that $\mathbf{B}_{h, N}(\omega, \lambda)$ depends non-linearly on the eigenvalue $\lambda$. This non-linearity can be studied in the same way as in section 4.3. Indeed, let $\mu_{m}^{h, N}(\omega, \lambda)$ denote the $m$-th eigenvalue $\mu$ of the following eigenvalue problem :

$$
\mathbf{B}_{h, N}(\omega, \lambda) U=\mu \mathbf{M}_{h} U
$$

so that, for $m=1,2 \ldots P$ :

$$
\mu_{m}^{h, N}(\omega, \lambda)=\underset{U^{(1)}, \ldots U^{(m-1)} \in \mathbb{R}^{P}}{M a x} \underset{\substack{U \in \mathbb{R}^{P}, U \neq 0 \\\left(U, U^{(k)}\right)=0, k=1, m-1}}{\operatorname{Min}} \frac{\left(\mathbf{B}_{h, N}(\omega, \lambda) U, U\right)}{\left(\mathbf{M}_{h} U, U\right)}
$$

Then problem (6.6) consists in solving the following family of fixed point equations for $m=1,2 \ldots P$ :

$$
\left\{\begin{array}{l}
\text { Find } \lambda \in \mathbb{R}, \lambda<-\beta_{\infty}^{2}(\omega), \text { such that : } \\
\mu_{m}^{h, N}(\omega, \lambda)=\lambda .
\end{array}\right.
$$

Using the min-max expression (6.8) of $\mu_{m}^{h, N}(\omega, \lambda)$, one can easily prove, as in section 4.3, that the functions :

$$
\lambda \longrightarrow \mu_{m}^{h, N}(\omega, \lambda)
$$

are continuous and non-increasing. Consequently, for each $m, m=1,2 \ldots P$, problem (6.9) 
has at most one solution $\lambda_{m}^{h, N}$ (see figure 16).

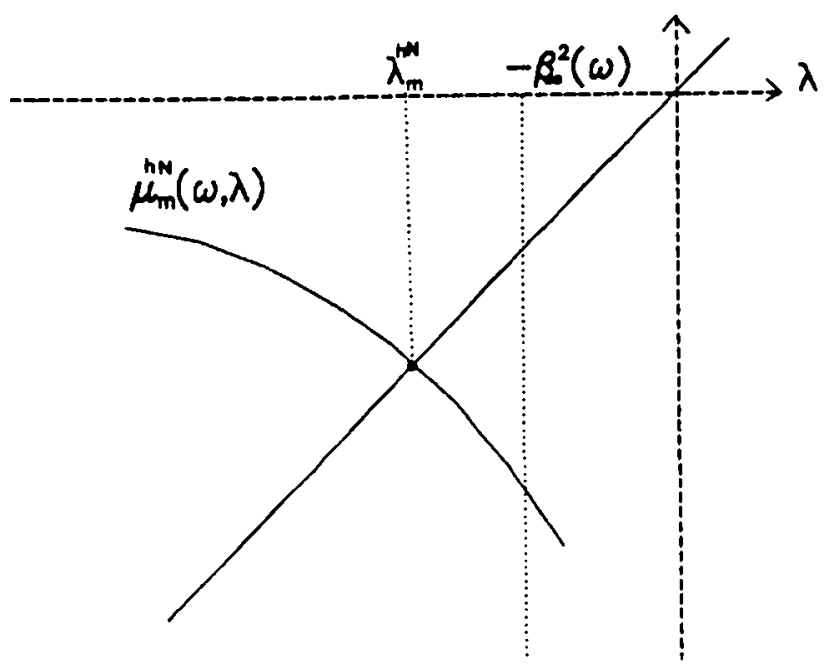

Figure $16:$ the curves $\lambda \longrightarrow \mu_{m}^{h, N}(\omega, \lambda)$

\subsubsection{The algorithm}

To solve problem (6.6), we use an iterative procedure based on a fixed point method.

Suppose that, at step $p$, we have computed an approximation $\lambda_{m}^{(p)}$ of $\lambda_{m}^{h, N}$. Then, the step $(p+1)$ can be decomposed into three stages :

1- Compute the matrix $\mathbf{B}_{h, N}\left(\omega, \lambda^{(p)}\right)$

2- Compute $\mu_{m}^{h, N}\left(\omega, \lambda^{(p)}\right)$

3- Set : $\lambda^{(p+1)}=\alpha \lambda^{(p)}+(1-\alpha) \mu_{m}^{h, N}\left(\omega, \lambda^{(p)}\right)$ where $\alpha$ is a relaxation parameter, $\alpha \in[0,1[$.

In the second step, we have to solve a linear eigenvalue problem of the form (6.7). We can use for example the inverse power method, with a shift equal to $\lambda^{(p)}$. If the eigenvalue we are looking for is very close to an other one, this method does not work and must be replaced by a method which computes simultaneously the whole cluster of eigenvalues (method of smultaneous iterations, Lanczos method...).

Thanks to the monotonicity of the function $\lambda \longrightarrow \mu_{m}^{h, N}(\omega, \lambda)$, the parameter $\alpha$ can always be choosen so that the process converges. However, the convergence can be very much improved by using a secant or a Newton type technique when the derivative $\frac{d}{d \lambda} \mu_{m}^{h, N}(\omega, \lambda)$ takes large values.

\subsection{Numerical results}

For the numerical computations we will present here, standard $P_{1}$ finite elements have been used. The value of $N$ is choosen equal to 5 and we have checked numerically that it is sufficient to compute the guided modes of low order.

Of course, the theoretical results obtained for a tangential beach cannot be illustrated numerically since such a domain cannot be meshed accurately and we have just considered the two other types of geometries. 


\subsubsection{The case of a cliff}

We have considered a shelf such that :

$$
h(x)=\left\{\begin{array}{lll}
.3 & \text { if } & x<3 \\
1 . & \text { if } & x>3
\end{array}\right.
$$

The abscissa of the artificial boundary $\Sigma$ is $d=3.5$. The mesh involves 467 triangles (cf. figure 17).

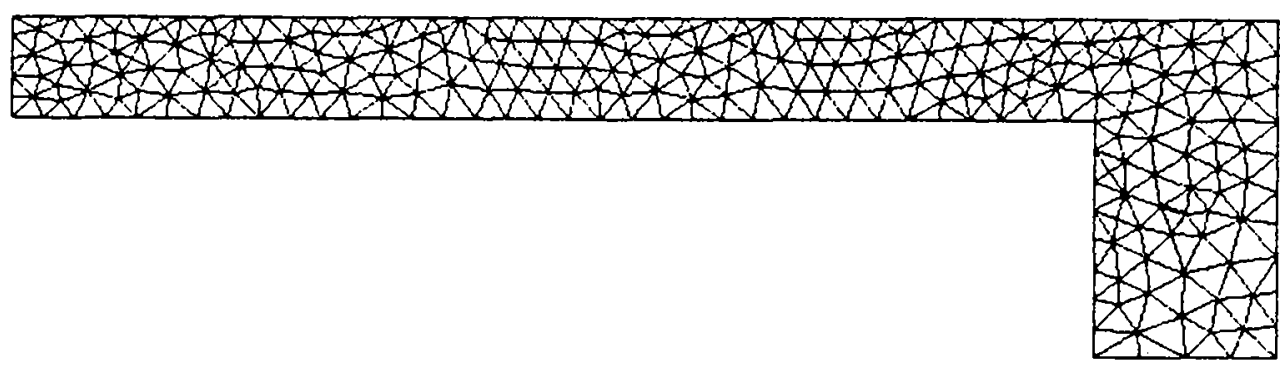

Figure 17

We have computed the first and the second modes. The corresponding values of $\omega$ and $\beta$ are :

\begin{tabular}{|c|c|}
\hline First mode & Second mode \\
\hline$\beta^{2}=2$ & $\beta^{2}=2$ \\
$\omega^{2}=.686$ & $\omega^{2}=1.012$ \\
\hline
\end{tabular}

The deformed free surfaces are represented on figure 18 (In fact, we have plotted the trace $\varphi(x, 0)$ of the potential on the free surface as a function of $\mathrm{x})$.

Notice that, according to the theory (see section 5.2.2), the free surface elevation for the 
fundamental mode does not take negative values.

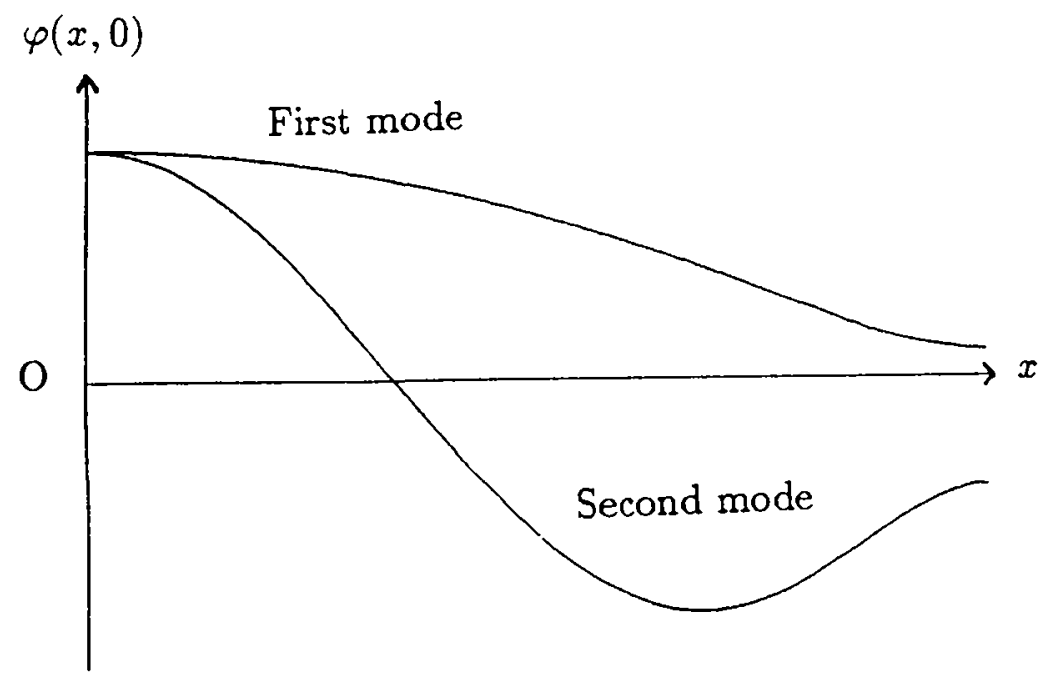

Figure 18

\subsubsection{The case of a sloping beach}

The general form of the relief which is considered here is :

$$
h(x)=\operatorname{Min}(1, x \tan \alpha) .
$$

The tests we have carried out concern the angles $\alpha=45^{\circ}$ and $\alpha=15^{\circ}$.

The case $\alpha=45^{\circ}$

The abscissa of the artificial boundary $\Sigma$ is $d=1.5$. The mesh involves 514 triangles (see figure 19).

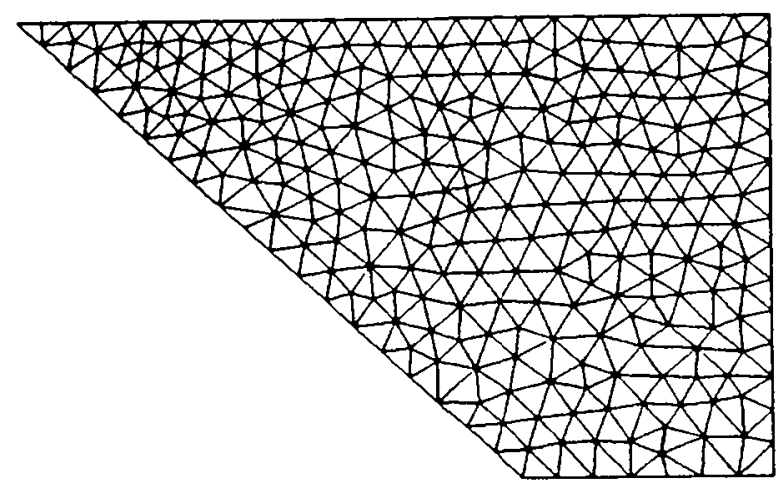

Figure 19 
According to the theory, there is only one guided mode which has been computed for 3 different values of the frequency :

\begin{tabular}{|c|c|}
\hline$\beta$ & $\omega^{2}$ \\
\hline 1. & .584 \\
2. & 1.144 \\
3. & 1.176 \\
\hline
\end{tabular}

The corresponding free surface elevations are plotted on figure 20. Notice that the mode becomes more and more confined as the frequency increases.

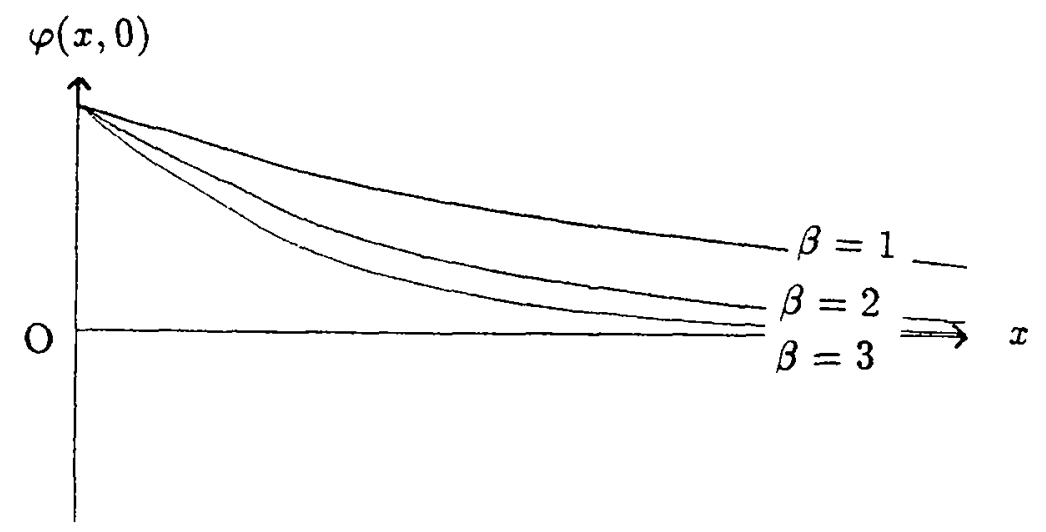

Figure 20

The case $\alpha=15^{\circ}$

The abscissa of the artificial boundary $\Sigma$ is $d=4.5$. The mesh involves 226 triangles (see figure 21).

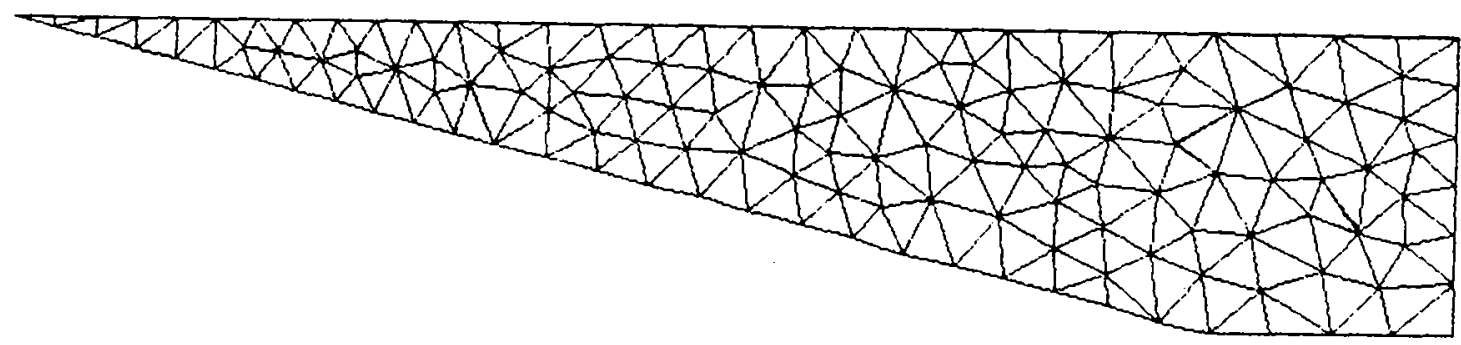

Figure 21 
According to the high frequency theory, we have found three guided modes for $\beta=2$ :

\begin{tabular}{|c|c|}
\hline Mode $\mathrm{n}^{\circ}$ & $\omega^{2}$ \\
\hline 1 & .38 \\
2 & 1.25 \\
3 & 1.84 \\
\hline
\end{tabular}

The corresponding free surface elevations are plotted on figure 22 .

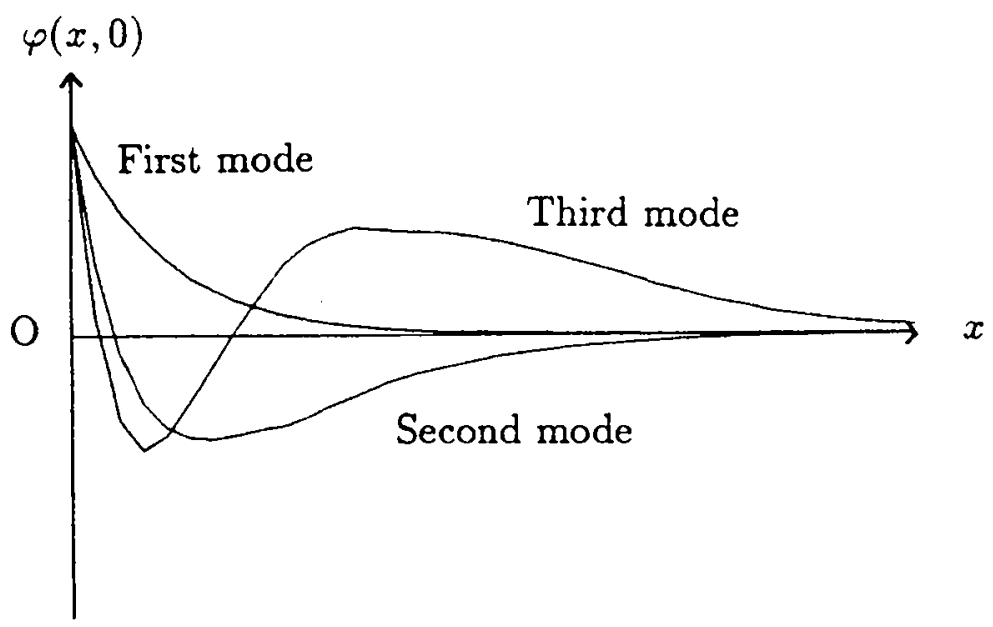

Figure 22 


\section{Annex}

We give here the analytical solution, obtained by separation of variables, of three eigenvalue problems set in a rectangular domain and which are referred to in sections 5.1 and 5.4 .

The rectangular domain $(R)$ is defined by :

$$
R=\left\{(x, y) \in \mathbb{R}^{2} ; 0<x<d,-h<y<0\right\}
$$

and we set :

$$
\begin{aligned}
& \left.\Gamma_{1}=\{0\} \cup\right]-h, 0[ \\
& \left.\Gamma_{2}=\right] 0, d[\cup\{-h\} \\
& \left.\Gamma_{3}=\{d\} \cup\right]-h, 0[ \\
& \left.\Gamma_{4}=\right] 0, d[\mathrm{U}
\end{aligned}
$$

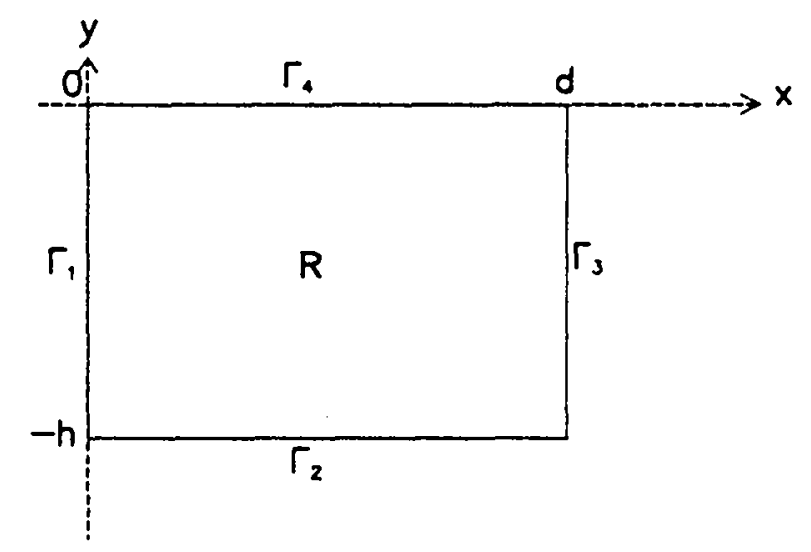

In the following, we will denote by $\beta(\omega)$ the unique positive solution $\beta$ of :

$$
\beta \tanh (\beta h)=\omega^{2}
$$

and by $\left(\nu_{n}(\omega)\right)_{n \geq 1}$ the sequence of positive solutions of :

$$
\nu \tanh (\nu h)=-\omega^{2}
$$

First, we consider two problems which are used for the study of the shelf in section 5.1. In that case, $h$ and $d$ denote respectively the depth above the shelf and the width of the shelf. 


\section{Problem 1 :}

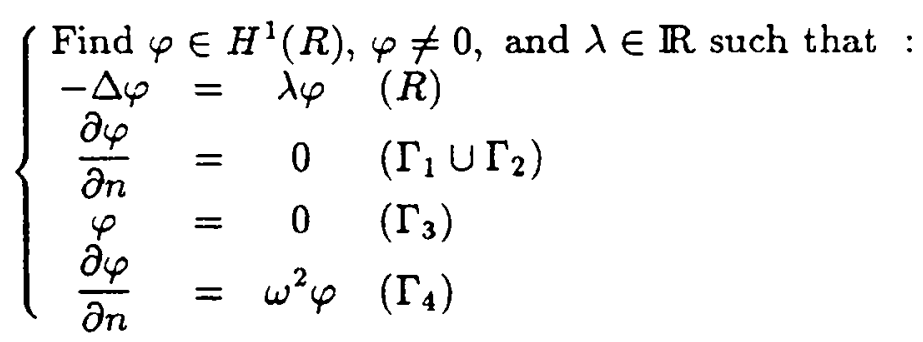

The set $\Sigma_{1}$ of eigenvalues of problem 1 is given by :

$$
\Sigma_{1}=\left\{\lambda_{m}^{(1)} ; m \in \mathbb{N}\right\} \cup\left\{\lambda_{m, n}^{(1)} ; m \in \mathbb{N}, n \in \mathbb{N}^{*}\right\}
$$

where :

- $\lambda_{m}^{(1)}=\left(m+\frac{1}{2}\right)^{2} \frac{\pi^{2}}{d^{2}}-\beta(\omega)^{2}$ is the eigenvalue associated to the eigenfunction

$$
\varphi_{m}^{(1)}=\cos \left(\left(m+\frac{1}{2}\right) \frac{\pi x}{d}\right) \cosh (\beta(\omega)(y+h))
$$

- $\lambda_{m, n}^{(1)}=\left(m+\frac{1}{2}\right)^{2} \frac{\pi^{2}}{d^{2}}+\nu_{n}^{2}(\omega)$ is the eigenvalue associated to the eigenfunction

$$
\varphi_{m, n}^{(1)}=\cos \left(\left(m+\frac{1}{2}\right) \frac{\pi x}{d}\right) \cos \left(\nu_{n}(\omega)(y+h)\right)
$$

\section{Problem 2 :}

$$
\left\{\begin{array}{cl}
\text { Find } \varphi \in H^{1}(R), \varphi \neq 0, \text { and } \lambda \in \mathbb{R} \text { such that }: \\
-\Delta \varphi=\lambda \varphi(R) \\
\frac{\partial \varphi}{\partial n}=0 \quad\left(\Gamma_{1} \cup \Gamma_{2} \cup \Gamma_{3}\right) \\
\frac{\partial \varphi}{\partial n}=\omega^{2} \varphi\left(\Gamma_{4}\right)
\end{array}\right.
$$

In that case, the set $\Sigma_{2}$ of eigenvalues is given by :

$$
\Sigma_{2}=\left\{\lambda_{m}^{(2)} ; m \in \mathbb{N}\right\} \cup\left\{\lambda_{m, n}^{(2)} ; m \in \mathbb{N}, n \in \mathbb{N}^{*}\right\}
$$

where :

- $\lambda_{m}^{(2)}=\frac{m^{2} \pi^{2}}{d^{2}}-\beta(\omega)^{2}$ is the eigenvalue associated to the eigenfunction

$$
\varphi_{m}^{(2)}=\cos \left(\frac{m \pi x}{d}\right) \cosh (\beta(\omega)(y+h))
$$

- $\lambda_{m, n}^{(2)}=\frac{m^{2} \pi^{2}}{d^{2}}+\nu_{n}^{2}(\omega)$ is the eigenvalue associated to the eigenfunction

$$
\varphi_{m, n}^{(1)}=\cos \left(\frac{m \pi x}{d}\right) \cos \left(\nu_{n}(\omega)(y+h)\right)
$$


The third problem we will consider has been used for the study of the sloping beach with angle $\frac{\pi}{4}$ in section 5.4. For that problem we suppose that the rectangle is a square i.e. $h=d$. In the particular case studied in section 5.4 , we have $h=d=h_{\infty}$ and what we have denoted here by $\beta(\omega)$ is referred to as $\beta_{\infty}(\omega)$ in the paper.

\section{Problem 3 :}

$$
\begin{cases}\text { Find } \varphi \in H^{1}(R), \varphi \neq 0, \text { and } \lambda \in \mathbb{R} \text { such that : } \\ -\Delta \varphi=\lambda \varphi & (R) \\ \frac{\partial \varphi}{\partial n}=0 & \left(\Gamma_{2} \cup \Gamma_{3}\right) \\ \frac{\partial \varphi}{\partial n}=\omega^{2} \varphi & \left(\Gamma_{1} \cup \Gamma_{4}\right)\end{cases}
$$

In that case :

$$
\Sigma_{3}=\left\{\lambda^{(3)}\right\} \cup\left\{\lambda_{m}^{(3)} ; m \in \mathbb{N}^{*}\right\} \cup\left\{\lambda_{m, n}^{(3)} ; m, n \in \mathbb{N}^{*}\right\}
$$

where :

- $\lambda^{(3)}=-2 \beta(\omega)^{2}$ is associated to the eigenfunction

$$
\varphi^{(3)}=\cosh (\beta(\omega)(x+h)) \cosh (\beta(\omega)(y+h))
$$

- $\lambda_{m}^{(3)}=\nu_{m}^{2}(\omega)-\beta(\omega)^{2}$ has the multiplicity 2 and is associated to

$$
\left\{\begin{array}{l}
\varphi_{m}^{(3)}=\cosh (\beta(\omega)(x+h)) \cos \left(\nu_{m}(\omega)(y+h)\right) \\
\psi_{m}^{(3)}=\cosh (\beta(\omega)(y+h)) \cos \left(\nu_{m}(\omega)(x+h)\right)
\end{array}\right.
$$

- $\lambda_{m, n}^{(3)}=\nu_{m}^{2}(\omega)+\nu_{n}^{2}(\omega)$ has also the multiplicity 2 and is associated to

$$
\left\{\begin{array}{l}
\varphi_{m, n}^{(3)}=\cos \left(\nu_{m}(\omega)(x+h)\right) \cos \left(\nu_{n}(\omega)(y+h)\right) \\
\psi_{m, n}^{(3)}=\cos \left(\nu_{n}(\omega)(x+h)\right) \cos \left(\nu_{m}(\omega)(y+h)\right)
\end{array}\right.
$$




\section{References}

[1] A. Bamberger and A. S. Bonnet, Mathematical Analysis of the Guided Modes of an Optical Fiber, SIAM. J. Math. Anal., 21 (1990), pp. 1487-1510.

[2] A. Bamberger, Y. Dermenjian, and P. Joly, Mathematical Analysis of the Propagation of Elastic Guided Waves in Heterogeneous Media, J. of Differential Eq., 88 (1990), pp. 113-154.

[3] A. Bamberger, P. Joly, And M. Kern, Propagation of Elastic Surface Waves along a Cylindrical Cavity of Arbitrary Cross Section, R.A.I.R.O. Modél. Math. Anal. Numér., 25 (1991), pp. 1-30.

[4] A. S. Bonnet, Analyse Mathématique de la Propagation de Modes Guidés dans les Fibres Optiques, $\mathrm{PhD}$ thesis, Université Paris VI, 1988. Thèse.

[5] A. S. Bonnet, F. Hemez, And P. Joly, Etude de la propagation de la houle le long des côtes, in Deuxième Colloque Maghrébin sur les Modèles numériques de l'ingénieur, Rabat,Maroc, 1989.

[6] A. S. Bonnet-Bendhia and F. Starling, Diffraction et ondes guidées pour des modes TE ou TM dans les réseaux électromagnétiques, tech. rep., Ecole Polytechnique, CMAP, 1991. Rapport Interne $n^{\circ} 243$.

[7] R. Djellouli, Contributions à l'Analyse Mathématique et au Calcul des Modes Guidés des Fibres Optiques, $\mathrm{PhD}$ thesis, Universté Paris XI, 1988. Thèse.

[8] D. V. EVANS AND P. MCIVER, Edge waves over a shelf : full linear theory, J. Fluid Mech., 142 (1984), pp. 79-95.

[9] N. Gmati, PhD thesis, Université Paris VI, 1992. Thèse.

[10] R. Grimshaw, Edge waves : a long-wave theory for oceans of finite depth, J. Fluid Mech., 62 (1974), pp. 775-791. Part 4.

[11] J. C. Guillot, Existence and Uniqueness of a Rayleigh Wave Propagating along the Free Boundary of a Transversally Isotropic Elastic Half Space, Math. Meth. in the Appl. Sci., 8 (1986), pp. $289-310$.

[12] F. John, On the motions of floating bodies . II, Comm. Pure Appl. Math., 3 (1950), p. $45-101$.

[13] P. Joly And R. Weder, New Results for Guided Waves in Elastic Heterogeneous Media, Math. Meth. in the Appl. Sci., (To appear).

[14] D. S. JONES, The eigenvalues of $\nabla^{2} u+\lambda u=0$ when the boundary conditions are given of semi-infinite domains, Proc. Camb. Phil. Soc., 49 (1953), pp. 668-684. 
[15] T. Kato, Perturbation Theory for Linear Operators, Springer, 1966.

[16] Laurentiev and Chabat, Effets hydrodynamiques et modèles mathématiques, Editions MIR.

[17] M. LENOIR AND A. TOUNSI, The localized finite element method and its appication to the two-dimensional sea-keeping problem, SIAM J. Numer. Anal., 25 (1988), pp. 729 752.

[18] A. Manes and A. M. Micheletti, Un'estensione della teoria variazionale classica degli autovalori per operatori ellitici del secondo ordine, Bolletino U. M. I., 7 (1973), pp. 285-301.

[19] D. Marcuse, Theory of Dielectric Optical Waveguides, Academic Press, 1974.

[20] P. MCIVER AND D. V. EVANS, The trapping of surface waves above a submerged horizontal cylinder, J. Fluid Mech., 151 (1985), pp. 243-255.

[21] J. C. NEDELEC AND F. STARLING, Integral equation methods in a quasi-periodic diffraction problem for the time harmonic Maxwell's equations, tech. rep., Ecole Polytechnique, CMAP, 1988. Rapport Interne $n^{\circ} 179$.

[22] H. PICQ, Détermination et calcul numérique de la première valeur propre d'opérateurs de Schrödinger dans le plan, $\mathrm{PhD}$ thesis, Université Nice, 1982. Thèse.

[23] M. ReEd AND B. Simon, Methods of Modern Mathematical Physics, vol. 1 et 4, Academic Press, 1981,.

[24] M. J. SimON AND F. URSELL, Uniqueness in linearized two-dimensional water-waves problems, J. Fluid Mech., 148 (1984), pp. 137-154.

[25] G. StamPacchia, Le probleme de Dirichlet pour les équations elliptiques du second ordre à coefficients discontinus, Ann. Inst. Fourier, 15 (1965),' pp. 193-257.

[26] J. J. StokeR, Water Waves, Intersciences Publishers, 1957.

[27] G. C. Stokes, Report on recent researches in hydrodynamics, Britt. Assn. Rep., (1846).

[28] J. TLILI, Calcul numérique d'ondes de surface élastiques guidées par une fissure, $\mathrm{PhD}$ thesis, Université Paris VI, 1991. Thèse.

[29] F. Ursell, Trapping modes in the theory of surface waves, Proc. Camb. Phil. Soc., 47 (1951), pp. 347-358.

[30] —_, Edge Waves on a Sloping Beach, Proc. R. Soc. Lond., A214 (1952), pp. 79-97. 
[31] _ Mathematical Aspects of Trapping Modes in the Theory of Surface Waves, J. Fluid. Mech., 183 (1987), pp. 421-437.

[32] R. Weder, Absence of Eigenvalues of the Acoustic Propagator in Deformed Wave Guides, Rocky Mount.J. of Math., 18 (1988), pp. 495-503.

[33] J. V. Wehausen and E. V. Laitone, Surface Waves, Encyclopaedia of Physics, Springer Verlag, 1960.

[34] C. H. WIlcox, Sound Propagation in Stratified Fluids, Springer-Verlag, 1984.

[35] K. J. WiTsch, Examples of Embedded Eigenvalues for the Dirichlet Laplacian in Perturbed Waveguides, Math. Meth. in the Appl. Sci., 12 (1990), pp. 91-93. 


\title{
ANALYSE MATHEMATIQUE DE LA PROPAGATION DE VAGUES GUIDEES
}

\author{
Anne-Sophie BONNET-BEN DHIA ${ }^{1}$ and Patrick JOLY ${ }^{2}$
}

\begin{abstract}
Résumé
Dans cet article, nous menons une analyse mathématique approfondie du phénomène de guidage de vagues par la côte. Nous travaillons à partir du modèle mathématique déduit de la théorie linéarisée de la propagation de la houle. Nos résultats concernent essentiellement l'existence, le nombre et le comportement à haute et basse fréquence des modes guidés. Ces résultats généralisent très largement ceux de la littérature. Ils mettent en évidence une large variété de phénomènes sur lesquels la géométrie de la côte a une influence prépondérante. Nous les obtenons à l'aide de la théorie spectrale des opérateurs autoadjoints non bornés. Des résultats numériques sont présentés à la fin de l'article pour illustrer la théorie.
\end{abstract}

\section{$\underline{\text { Abstract }}$}

In this article, we present a detailed mathematical analysis of the phenomenon of water waves guided by the coast. We work with the mathematical model issued from the linearized theory of gravity waves. Our results mainly concern the existence, the number and the high and low frequency behaviours of the guided modes. These results point out a large variety of phenomena which are deeply influenced by the geometry of the coast. We obtain them with the help of the spectral theory of unbounded selfadjoint operators. Some numerical results are presented at the end of the paper to illustrate the theory.

Mots-clé

Ondes guidées - Propagation de la houle - Relation de dispersion - Théorie spectrale Principe du Min-Max.

Keywords principle.

Guided waves - Water waves propagation - Dispersion relation - Spectral theory - Min-Max

ENSTA-GHN URAD0853 du CNRS Centre de l'Yvette, Chemin de la Hunière, 91120 Palaiseau.

2 INRIA Domaine de Voluceau-Rocquencourt, BP 105, 78153 Le Chesnay Cédex. 

ISSN 0249-6399 